\title{
Gradient Flow of the Superconducting Ginzburg-Landau Functional on the plane
}

\author{
Sophia Demoulini \\ AND \\ DAVID STUART ${ }^{1}$
}

First we prove the existence of global smooth solutions of the gradient flow of the superconducting Ginzburg Landau (or Abelian Higgs) functional on $\mathbb{R}^{2}$. It is then proved that in the case of critical coupling, for a large class of initial data of arbitrary winding number $N$, each solution converges in temporal gauge to a unique solution of the static equations of the same winding number. The proof has two essential ingredients. Firstly a weighted energy identity is used to obtain spatial exponential decay of certain quantities uniformly in time. This implies the strong subsequential convergence to a static solution in the $H^{2}$ norm. Secondly, an adiabatic approximation in the neighbourhood of the static solution space is used to prove that the solution converges without passing to subsequences. Thus the $\omega$-limit set of each solution is a point. The adiabatic approximation consists of finding, at each time, the $L^{2}$-closest point to the solution on the space of static solutions of the same winding number as the initial data. Special cases of the result imply that two vortices of opposite sign will annihilate for a large class of initial data and that a single vortex is asymptotically stable with respect to a large class of perturbations.

\section{Table of Contents.}

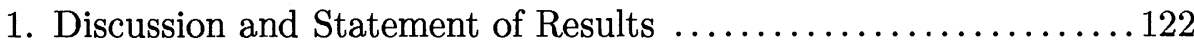

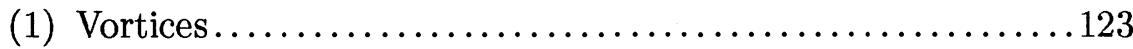

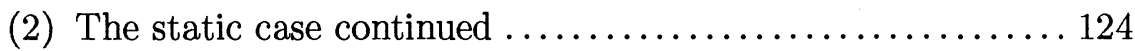

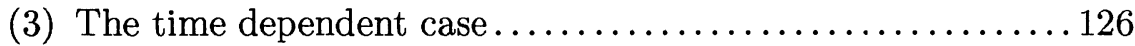

\footnotetext{
${ }^{1}$ Supported by NSF
} 
(4) Statement of Results ........................ 127

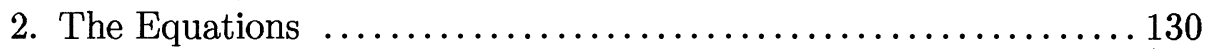

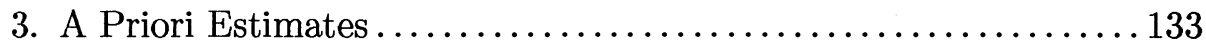

(1) Energy, Uniform Estimates and Exponential Decay ........ 133

(2) Estimates for Time Asymptotics ................. 141

4. Existence and Uniqueness $\ldots \ldots \ldots \ldots \ldots \ldots \ldots \ldots \ldots \ldots \ldots \ldots \ldots \ldots$

5. Large Time Asymptotics ................................ 148

(1) Extraction of Convergent Subsequence................. 149

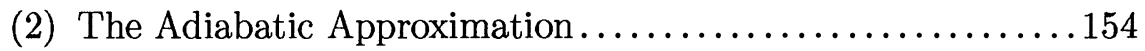

i. Gauge Orthogonality and Slices .............. 157

ii. The moduli Space...........................164

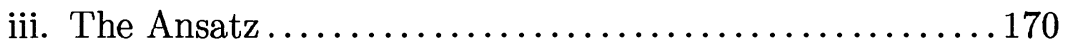

iv. Proof of the Adiabatic Approximation theorem........176

6. Proof of Main Theorem ........................... 184

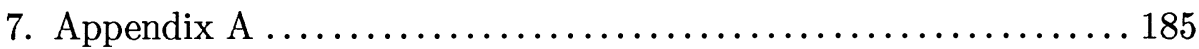

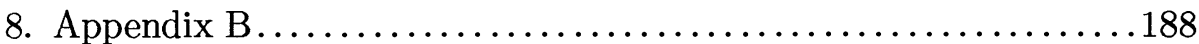

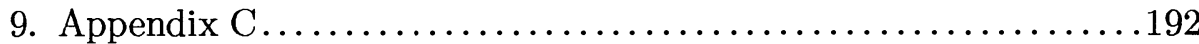

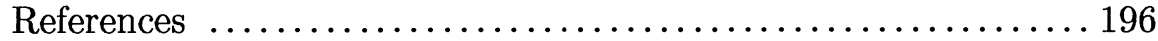

\section{Discussion and Statement of Results.}

The action for the Abelian Higgs, or superconducting Ginzburg-Landau, functional in two dimensions is given by

$$
\begin{aligned}
\mathcal{E}(A, \Phi) & =\frac{1}{2} \int_{\mathbb{R}^{2}} F_{A} \wedge * F_{A}+D_{A} \Phi \wedge * D_{A} \Phi+\frac{\lambda}{4} *\left(|\Phi|^{2}-1\right)^{2} \\
& =\frac{1}{2} \int_{\mathbb{R}^{2}}|B|^{2}+\left|D_{A} \Phi\right|^{2}+\frac{\lambda}{4}\left(|\Phi|^{2}-1\right)^{2} d x^{1} \wedge d x^{2}
\end{aligned}
$$

Here $*$ is the Hodge duality operator, $-i F_{A}$ is the curvature of an $S^{1}$ connection $-i A$ and $\Phi$ is a section of the associated complex line bundle. The induced connection couples $A$ and $\Phi$ via the covariant derivative $D_{A}=d-i A$. 
Thus $A$ is a real one-form and $F_{A}=d A$ is a real two-form. The function $B=* F_{A}$ is known as the magnetic field, while $\Phi$ is called either the order parameter or the Higgs field. The action is invariant under an infinite dimensional gauge group of symmetries $\mathcal{G} \equiv C^{\infty}\left(\mathbb{R}^{2} ; S^{1}\right)$ of smooth gauge transformations,

$$
e^{i \chi}: \mathbb{R}^{2} \longrightarrow S^{1}
$$

acting on $(A, \Phi)$ by

$$
\rho(\chi):(A, \Phi) \mapsto\left(A+\nabla \chi, \Phi e^{i \chi}\right) .
$$

The quantities $B,|\Phi|,\left|D_{A} \Phi\right|$ are gauge invariant. The moduli space is defined as the space of gauge equivalence classes of solutions to the variational equations associated with the action. These variational or static equations are

$$
\begin{aligned}
-\Delta_{A} \Phi & =\frac{\lambda}{2} \Phi\left(1-|\Phi|^{2}\right) \\
d^{*} d A & =\left(i \Phi, D_{A} \Phi\right)
\end{aligned}
$$

where $d^{*}$ is the adjoint operator of $d$ and $d^{*}=-* d *$. The last equation can also be written as $d B=-*\left(i \Phi, D_{A} \Phi\right)$. It is proved in [19] that solutions $(A, \Phi)$ of finite action satisfy $\lim _{|x| \rightarrow \infty}\left(|\Phi|,\left|D_{A} \Phi\right|,|B|\right)=(1,0,0)$, in fact exponentially fast. Because these limits are sufficiently uniform it can be shown that the vortex number or winding number,

$$
N=\frac{1}{2 \pi} \int_{\mathbb{R}^{2}} F_{12} d x^{1} \wedge d x^{2}=\frac{1}{2 \pi} \int_{\mathbb{R}^{2}} B d x^{1} \wedge d x^{2}
$$

coincides with the homotopy class $[\Phi]$ in $\pi_{1}\left(S^{1}\right)$ and is thus an integer (cf. [JT]). It is commonly called charge. Except for a bounded region in space, finite action solutions are close to their asymptotic (in space) values where $|B|=\left|D_{A} \Phi\right|=1-|\Phi|^{2}=0$.

We now give some physical background to the problem. Following this we return to the static problem and then discuss the time dependent case before stating the results in section 1.d.

\subsection{Vortices.}

Strictly, vortices are defined as finite action solutions of the static variational equations (1.2) (c.f. [JT]) and the term refers mostly to non-constant finite action solutions. It can be shown that when $|\Phi|$ is bounded away from zero then the solution is constant up to gauge equivalence, that is $|\Phi| \equiv 1$ 
and $B \equiv 0$. In all other cases, $\Phi$ must have zeros. In the event that these are isolated they may be thought of as the "centers" of vortex solutions and thus the term vortices also refers to the zeros of $|\Phi|$. Thus the term "vortex" will be used to refer to a zero of $\Phi$ (for all $\lambda$ ) as well as to a non-constant finite action configuration. A multivortex solution refers to the case in which $\Phi$ has more than one zero, interpreted as a nonlinear superposition of single vortices. For solutions of the time dependent model, which we discuss below, we will see that the vortex number at all times will be equal to that of the initial data. In that case "vortex" may also refer to the location of the zeros of the time dependent density $\Phi$. Furthermore, "vortex" is often used only for a positive vortex number (positive charge) and "antivortex" is used in the case of a negative vortex number. Vortices have been observed in certain kinds of superconductors in the presence of applied magnetic fields.

According to a heuristic interpretation of vortices they interact as particles. As such they repel or attract and forces must balance for a static configuration. It is postulated for physical reasons that vortices of opposite charge attract for all values of $\lambda$ (the strength of the force varying as $\lambda$ varies). Vortices of the same charge are expected to attract for $\lambda<1$ and repel for $\lambda>1$, while at the critical value $\lambda=1$ there is no net force between vortex-vortex pairs which then do not interact. The attraction or repulsion of like vortices is induced by the competition between the force induced by the vector potential (under which vortices behave like electric charges) and the force induced by the scalar potential (which induces an attractive force regardless of the sign of the charges). For like vortices the attraction and repulsion balance out exactly at the value $\lambda=1$. Computer studies by Jacobs and Rebbi ([23]) gave the original support to this interpretation and Taubes' existence result discussed below confirms it for the case $\lambda=1$. The parameter $\sqrt{\lambda}$ is the ratio of the rates at which $\Phi$ and the magnetic field $B$ approach their asymptotic values as $|x| \rightarrow \infty$. These rates are equal when the forces cancel at $\lambda=1$. (This is discussed in detail in the book [19]). Also there is a formula, which we give below, for the potential energy between two vortices if $\lambda$ is close to 1 ([37]) which supports this picture ([35]).

\subsection{The static case continued.}

The static case $\lambda=1$ is best understood due to the fact that the functional is then a symmetric reduction of the four dimensional Yang-Mills functional. All critical points are minima and solve (for $N>0$ ) the follow- 
ing first order equations $([9],[19])$ :

$$
\begin{gathered}
B=\frac{1}{2}\left(1-|\Phi|^{2}\right) \\
D_{A} \Phi-i * D_{A} \Phi=0,
\end{gathered}
$$

which will be written schematically as $\mathcal{B}(A, \Phi)=0$. These can be reduced to a single elliptic equation for the unknown $u=\ln |\Phi|^{2}$,

$$
-\Delta u+e^{u}-1=-4 \pi \sum_{k=1}^{N} \delta\left(z-Z_{k}\right)
$$

for $Z_{1}, \ldots, Z_{N}$ fixed points in the complex plane, the singularities of $u$ which are precisely the zeros of $\Phi$, or the vortex sources. It is shown in [19] that when $\lambda=1$ any finite action critical point is characterized completely by its zeros; in particular any finite action solution has finitely many isolated zeros (the centers of the vortices) and is real analytic (in an appropriate gauge). In addition all critical points are minima. The action equals $\pi|N|$ so in the case of zero winding number there is an essentially unique minimizer which modulo gauge transformations satisfies $|\Phi|=1$ and $A=0$.

For other values of $\lambda$ less is proved but there are conjectures and the a priori estimates in [19] show that all finite action critical points have the same regularity as in the case $\lambda=1$. In addition it is expected that, similarly to the case of $\lambda=1$, vortices occur as the isolated zeros of $\Phi$ with behaviour consistent with the heuristic interpretation of vortices as interacting particles described above. In the papers [37, 39] it was proved that the potential energy between two like vortices is given approximately for $\lambda$ close to 1 by

$$
V(a)=\frac{\lambda-1}{8} \int_{\mathbb{R}^{2}}\left(e^{u}-1\right)^{2} d x
$$

where $u$ is the solution of (1.3) with $Z_{1}=-\frac{a}{2}$ and $Z_{2}=\frac{a}{2}$. This was computed in the paper [35] and was found to be a monotonic function of $a$. This confirms the conjecture that the vortices attract for $\lambda<1$ and repel for $\lambda>1$. Thus, for $\lambda<1$ and each $N$, it is conjectured that there is a unique (up to gauge equivalence and translations in $\mathbb{R}^{2}$ ) critical point which is a minimum of the action and is spherically symmetric (in an appropriate sense) around the origin where it possesses a zero of order $N$. Similarly it is conjectured in the case of $\lambda>1$, that there is a critical point, a minimum, which is stable if $N=0, \pm 1$ and unstable in the case of $|N|>1$, in which case no stable finite action solutions exist. The idea is that like vortices 
repel so a critical point can occur only with all vortices coinciding (i.e. their zeros at the same point) which is an unstable configuration of the solution for $|N|>1$ but is stable otherwise. For all $\lambda>0$ it can be shown that if $\Phi$ has no zeros there is a gauge transformation in which the connection $A=0$ and $\Phi$ is a constant of modulus one. The same result in the more general situation of zero winding number has been proved only in the case $\lambda=1$.

\subsection{The time dependent case.}

Time dependent models corresponding to (1.1) have been considered in two situations: firstly the Lorentz invariant equations studied in [37] for values of the parameter $\lambda$ near the critical value 1 . In this paper we consider the gradient flow equations associated to (1.1). This is a special case of the equations which are of physical interest in superconductivity (see for example $[10,5])$. Thus we consider the equations

$$
\partial_{t}(A, \Phi)=-\operatorname{grad} \mathcal{E}
$$

There is a slightly more general form of time dependent equations obtained by introducing a time component $A_{0}$ to the connection and by differentiating covariantly in time according to $D_{A_{0}}=\partial_{t}-i A_{0}$. These equations are

$$
\begin{aligned}
D_{A_{0}} \Phi-\Delta_{A} \Phi & =\frac{\lambda}{2} \Phi\left(1-|\Phi|^{2}\right) \\
\dot{A}-\nabla A_{0}+d^{*} d A & =(i \Phi, D \Phi) .
\end{aligned}
$$

The gauge group now consists of functions which depend upon the time variable as well. Particular gauges are of significance: the case of $A_{0}=0$ is called the temporal gauge and in this gauge we recover (1.4); then there is the Coulomb gauge in which $\nabla \cdot A=0$ and the uniformly parabolic gauge in which $A_{0}=-\nabla \cdot A$. In the next section we consider all these and write the equations in each gauge. The conjectures on the variational problem translate into the following conjectures for the gradient flow which in the case of $\lambda \neq 1$ follow from the analysis in [39]:

(i) If $\lambda<1$ all solutions of the gradient flow equations converge uniformly to a finite action critical point which is a radially symmetric vortex with winding number equal to that of the initial data.

(ii) If $\lambda=1$ solutions of the gradient flow converge uniformly to one of Taubes' multi-vortex solutions. This is the main result of the present paper. (iii) If $\lambda>1$ then if the winding number is either 0 or \pm 1 the solution converges uniformly to a finite action critical point which is a symmetric 
vortex with winding number equal to that of the initial data. If the winding number is greater than one there will not generically be uniform convergence due to the escape of vortices to infinity; however, convergence on compact sets may still persist.

Most previous work on these equations seems to have been on bounded domains. There has been much work on the $\lambda \rightarrow \infty$ limit of the equations $([25,22])$. In $[33,32]$ the stability and symmetry of minimisers is discussed. For the case of unbounded domains a related paper is [1] where the equations with no connection present $(A \equiv 0)$ are studied. Thus the static action in this case becomes

$$
\mathcal{G L}(u)=\int \frac{|\nabla u|^{2}}{2}+\frac{1}{4}\left(|u|^{2}-1\right)^{2}
$$

for $u(x) \in \mathbb{C}$. The authors prove that for appropriate initial data of zero winding number $u$ converges uniformly to a constant. Thus in this case there is vortex annihilation; this means that vortices of opposite charge will eventually merge and annihilate. As mentioned above, vortex annihilation remains open for the full Ginzburg-Landau gradient flow equations (1.4) for general $\lambda>0$. However, our main result implies vortex annihilation for a large class of initial data and for $\lambda=1$. The question of stability and symmetry of the static vortices for $\mathcal{G L}$ on $\mathbb{R}^{2}$ has been considered in [31], where it is shown that if the winding number is greater than one the radial vortices are linearly unstable, while they are linearly stable otherwise. In the paper [40] asymptotic stability of vortices in the gradient flow of $\mathcal{G L}$ is established. Again our results imply this for the full system (1.4) with $\lambda=1$ for a large class of perturbations. In a relevant context, Hassell [18] has proved existence and time asymptotic convergence to a solution of the static equations for the heat flow of the Yang-Mills-Higgs equations in the case of critical coupling; we use here a different method to his.

\subsection{Statement of Results.}

In the remainder of the paper we first describe the equations for the gradient flow in the gauges mentioned above and prove existence and uniqueness of a global $C^{\infty}$ solution for all $\lambda>0$. This is based on a priori $L^{\infty}$ estimates for $B$ and $D \Phi$ and their derivatives using a method of Hamilton as adapted by Hassell ([18]). By a theorem for parabolic systems stated in the appendix, solutions decay exponentially as $|x| \rightarrow \infty$. Next we discuss time asymptotics. We show $L^{1}\left(\mathbb{R}^{2}\right)$ estimates for $B, D \Phi$ and $|\Phi|$ independent of time. Using the maximum principle we show that these quantities decay 
exponentially as $|x| \rightarrow \infty$ and in the case of $\lambda=1$ this decay is uniform with respect to time for a large class of initial data. This depends on a Pohozaev type identity. Following this we show that in the Coulomb gauge $(A, \Phi)$ converges subsequentially for all $\lambda>0$ weakly in $L^{p}\left(\mathbb{R}^{2}\right)$ for $p>2$ and also in $C^{\infty}\left(\mathbb{R}^{2}\right)$ in the topology of convergence on compact sets to a solution of the static equations. For $\lambda=1$ this convergence is improved to a strong convergence in $L^{2}$, using the Pohozaev type identity. It is then shown that in an appropriate (time-dependent) gauge the solution gets arbitrarily close in $H^{2}$ to a solution of the static equations.

So far this convergence is subsequential; the next stage is to show that we have genuine convergence without passing to subsequences i.e., that the $\omega$-limit set of the solution is a point. There is a general theorem due to $\mathrm{L}$. Simon which asserts that this is true for gradient flows of real analytic functionals on compact manifolds satisfying a weak convexity condition ([36]). In this paper we are working on an unbounded domain $\mathbb{R}^{2}$ so this does not apply directly. We will use a different, geometrical method to prove the existence of a limit. This method, which seems to have more similarity with the methods of [7], is often called an adiabatic approximation, and amounts to tracking the point on the space of static solutions which is closest to the solution at each time. This is done via a tubular neighbourhood construction in theorem 5.15. (This is nonstandard since we find the closest point in the $L^{2}$ norm while the manifold structure is induced from the $H^{2}$ topology.) In theorem 5.4 it is shown that for $\lambda=1$ and when the solution is sufficiently close in $H^{2}$ to moduli space, it converges to a unique static solution. This is inspired by Manton's method ([27]) which has been extensively used in a similar context to study scattering of vortices and monopoles. For the Lorentz invariant Abelian Higgs equations this was justified in [37] and for the Yang-Mills-Higgs equations in [38].

We now state the main theorems which are proved in this paper. Introduce the notation

$$
\Psi=(A, \Phi) \quad \mathcal{U}=\left(B, D_{A} \Phi, D_{A}^{2} \Phi, \nabla B, w\right)
$$

where $B=* d A$ and $w=\frac{1}{2}\left(1-|\Phi|^{2}\right)$ and for $r>0, N \in \mathbb{Z}$ define the class of functions

$$
\begin{aligned}
\mathcal{C}^{r, N} \equiv\left\{(\Psi, \mathcal{U}) \in C^{\infty}\left(\mathbb{R}^{2}\right): \quad\right. & 0 \leq|\Phi|<1,|\Phi| \text { has winding number } \mathrm{N} \\
& \exists M>0 \text { such that }|\mathcal{U}| \leq M e^{-r|x|} \\
& \Psi, \mathcal{U} \text { and all their derivatives are bounded }\} .
\end{aligned}
$$

Theorem A Given initial data $\Psi(0), \mathcal{U}(0)$ in $\mathcal{C}^{r, N}$ there exists a unique solution $\Psi(x, t) \in C^{\infty}\left(\mathbb{R}^{2} \times(0, \infty)\right) \cap C\left([0, \infty) ; L^{\infty}\left(\mathbb{R}^{2}\right)\right)$ of (1.4) (see also 
(2.5) below) with $\lambda>0$ such that

(i) $\lim _{t \rightarrow 0}\left(\|\Psi(t)-\Psi(0)\|_{L^{\infty}}+\|\mathcal{U}(t)-\mathcal{U}(0)\|_{L^{\infty}}\right)=0$

(ii) $\|\mathcal{U}(t)\|_{L^{1}} \leq c$ and $\left\|D_{A}^{\alpha} \mathcal{U}(t)\right\|_{L^{\infty}} \leq c(\alpha)$ for all $t>0$ and all multiindices $\alpha$. (Here it is to be understood that the quantities $D_{A} \Phi, D_{A}^{2} \Phi$ are to be differentiated covariantly with $D_{A}$ while $B, \nabla B, w$ are to be differentiated in the usual way.)

This is proved in section 3, theorem 4.2 using theorems 3.6 and 3.9.

Theorem B Consider initial data as in theorem $A$ which in addition satisfy

$$
\begin{gathered}
|B(x, 0)| \leq \frac{1}{2}\left(1-|\Phi(x, 0)|^{2}\right) \\
\left|D_{A(0)} \Phi(x, 0)\right| \leq \frac{3}{2}\left(1-|\Phi(x, 0)|^{2}\right) .
\end{gathered}
$$

Then,

(i) for $\lambda \leq 1$, for all $\epsilon \in(0,1)$ and for all $T>0$ there exists $M(\epsilon, T)>0$ such that for $0 \leq t \leq T$

$$
|\mathcal{U}(x, t)| \leq M(\epsilon, T) e^{-\sqrt{\lambda}(1-\epsilon)|x|} ;
$$

(ii) for $\lambda=1$ there exists a constant $c$ depending only on the initial data such that:

$$
\sup _{t \geq 0} \int_{\mathbb{R}^{2}}|x|^{2}\left(|B|^{2}+|D \Phi|^{2}+\frac{\lambda}{4}\left(1-|\Phi|^{2}\right)^{2}\right) d x \leq c
$$

and in (1.7) we can take $M(\epsilon, T)=M_{\epsilon}$, i.e. the exponential decay is uniform with respect to time.

This is proved in theorems 3.8, 3.11 and 3.2 in section 3. (The existence theorem 4.2 asserts that these a priori estimates hold for our solution).

The following is our main result:

Main Theorem For initial data as in theorem $B$ there exists a finite action critical point $\Psi_{\infty}=\left(A_{\infty}, \Phi_{\infty}\right)$ of winding number $N$ such that $(\Psi(t)-$ $\left.\Psi_{\infty}\right) \rightarrow 0$ in $H^{2}\left(\mathbb{R}^{2}\right)$ as $t \rightarrow \infty$. In addition $\Psi_{\infty}$ can be obtained as follows: there exists a time $T$ such that for each $t>T$ there exists a unique finite action critical point of $\mathcal{E}$ of winding number $N$ which is $L^{2}$-closest to $\Psi(t)$. If we call this critical point $\mathbb{P}(\Psi(t))$ then there exists a constant $\delta>0$, depending only on the winding number, such that

$$
\left(\|\Psi(t)-\mathbb{P}(\Psi(t))\|_{H^{2}}+\left\|\Psi_{\infty}-\mathbb{P}(\Psi(t))\right\|_{H^{2}}\right) \leq C e^{-\delta t}
$$


where $C$ depends on the initial data.

The proof of this theorem appears in section 6 .

Remarks. (i) Notice that the convergence is taking place in the temporal gauge.

(ii) The condition (1.5) is essential in our approach, but (1.6) could be removed. It is interesting to find out whether (1.5) is really essential or not.

(iii) The solution is also expected to decay exponentially in space for $\lambda>1$. However this decay is conjectured to be uniform in time only for $\lambda \leq 1$. (iv) The final statement in the main theorem illustrates our method of proof of the existence of a limit: we find at each time the critical point $\mathbb{P}(\Psi(t))$ which is closest to $\Psi(t)$ and show that these approach one another exponentially fast. We then show $\mathbb{P}(\Psi(t))$ approaches a limit exponentially fast to complete the proof of the theorem.

\section{The equations.}

We now write down the equations in the various gauges which will be needed in this work. We will use the following inner product for complex numbers:

$$
(a, b)=\frac{\bar{a} b+a \bar{b}}{2} .
$$

We recall the Ginzburg-Landau functional (1.1)

$$
\mathcal{E}(A, \Phi)=\frac{1}{2} \int_{\mathbb{R}^{2}}|B|^{2}+\left|D_{A} \Phi\right|^{2}+\frac{\lambda}{4}\left(|\Phi|^{2}-1\right)^{2} d x^{1} \wedge d x^{2}
$$

where in the notation used in the introduction $B=\left(\partial_{1} A_{2}-\partial_{2} A_{1}\right)$ so $B d x_{1} \wedge$ $d x_{2}=d A$ and $D_{A_{j}} \Phi=D_{j} \Phi=\partial_{j} \Phi-i A_{j} \Phi$. Even though the (covariant) differential operator $D$ depends on the connection $A$, this dependence will be usually supressed. We will let $\Delta_{A} \Phi=D_{1}^{2} \Phi+D_{2}^{2} \Phi$ and $\Delta$ denote the usual Laplacian. Here $d$ is the exterior derivative and $d^{*}$ is its adjoint, so that $d^{*}\left(F d x_{1} \wedge d x_{2}\right)=\partial_{2} F d x_{1}-\partial_{1} F d x_{2}=\partial_{1}^{*} F d x_{1}+\partial_{2}^{*} F d x_{2}$. Introducing a time component $A_{0}$ to the connection $A$ and a corresponding covariant derivative $D_{0} \Phi=\dot{\Phi}-i A_{0} \Phi$, where $\dot{\Phi}=\frac{d}{d t} \Phi$, we obtain the fully gauge invariant gradient flow Abelian Higgs equations

$$
\begin{aligned}
D_{0} \Phi-\Delta_{A} \Phi & =\frac{\lambda}{2} \Phi\left(1-|\Phi|^{2}\right) \\
\dot{A}-\nabla A_{0}+d^{*} d A & =(i \Phi, D \Phi) .
\end{aligned}
$$

These equations are invariant under the gauge group of symmetries $e^{i \chi} \in$ $C^{\infty}\left(\mathbb{R}^{2} \times \mathbb{R}^{+} ; S^{1}\right)$, that is, for any real smooth function $\chi(x, t)$ the transfor- 
mation $\rho(\chi)$ (often referred to as the gauge transformation)

$$
\Phi \rightarrow e^{i \chi} \Phi \quad A \rightarrow A+\nabla \chi \quad A_{0} \rightarrow A_{0}+\dot{\chi}
$$

takes solutions into solutions. To check that this is a symmetry of the equations, notice that

$$
D_{j} \Phi \rightarrow e^{i \chi} D_{j} \Phi \quad D_{0} \Phi \rightarrow e^{i \chi} D_{0} \Phi .
$$

The equations form a degenerate parabolic system. The fully invariant equations transform under specific gauges to useful forms and there are various ways to factor gauge equivalence out. Firstly, we obtain the heat flow equations $\partial_{t}(A, \Phi)=-$ gradE which is the special choice of

$$
A_{0}=0 \text {. }
$$

This is called the temporal gauge, which is clearly always attainable by applying the gauge transformation determined by $\chi(x, t)$ where

$$
\dot{\chi}+A_{0}=0
$$

In this gauge the equations are

$$
\begin{aligned}
\dot{\Phi}-\Delta_{A} \Phi & =\frac{\lambda}{2} \Phi\left(1-|\Phi|^{2}\right) \\
\dot{A}+d^{*} d A & =(i \Phi, D \Phi)
\end{aligned}
$$

where the dot means differentiation with respect to time.

There are other useful gauges; in particular there is the Coulomb (or Hodge) gauge in which

$$
\nabla \cdot A=0
$$

This is obtained by applying the gauge transformation determined by $\chi$ where

$$
\Delta \chi+\nabla \cdot A=0
$$

In this gauge we can write the equations as a coupled elliptic parabolic system:

$$
\begin{aligned}
D_{0} \Phi-\Delta_{A} \Phi & =\frac{\lambda}{2} \Phi\left(1-|\Phi|^{2}\right) \\
\dot{A}-\Delta A-\nabla A_{0} & =(i \Phi, D \Phi) \\
-\Delta A_{0} & =\left(i \Phi, D_{0} \Phi\right)
\end{aligned}
$$


Another useful gauge is the uniformly parabolic gauge in which we require that

$$
\nabla \cdot A-A_{0}=0 \text {. }
$$

This is obtained by applying the gauge transformation determined by $\chi$ where

$$
\dot{\chi}-\Delta \chi=\nabla \cdot A-A_{0}
$$

This gauge is useful because the equations then form a uniformly parabolic system for $(A, \Phi)$ :

$$
\begin{aligned}
\dot{\Phi}-\Delta_{A} \Phi-i \Phi \nabla \cdot A & =\frac{\lambda}{2} \Phi\left(1-|\Phi|^{2}\right) \\
\dot{A}-\Delta A & =(i \Phi, D \Phi) .
\end{aligned}
$$

Let us write these in a general form by introducing $\Psi=\left(A_{1}, A_{2}, \Phi_{1}, \Phi_{2}\right)$, where $\Phi_{1}, \Phi_{2}$ are the real and imaginary parts of $\Phi$. Then $\Psi$ satisfies an equation of the form

$$
\dot{\Psi}-\Delta \Psi=f(\Psi, \nabla \Psi)
$$

where $f(\Psi, Z)$ is cubic with respect to $\Psi$ and linear with respect to $Z$.

We will also need some identities obtained by differentiating the full equations. Differentiating once we obtain

$$
\dot{B}-\Delta B+|\Phi|^{2} B=2\left(i D_{1} \Phi, D_{2} \Phi\right)
$$

$$
\left(D_{0}-\Delta_{A}\right) D \Phi+2 i B D^{*} \Phi+i \Phi(i \Phi, D \Phi)+\lambda \Phi(\Phi, D \Phi)=\frac{\lambda}{2} D \Phi\left(1-|\Phi|^{2}\right)
$$

where

$$
D^{*} \Phi=D_{2} \Phi d x_{1}-D_{1} \Phi d x_{2}=D_{1}^{*} \Phi d x_{1}+D_{2}^{*} \Phi d x_{2} .
$$

Differentiating a second time we find

$$
\left(\partial_{t}-\Delta\right) \nabla B+|\Phi|^{2} \nabla B=-2(\Phi, D \Phi) B+2 \nabla\left(i D_{1} \Phi, D_{2} \Phi\right)
$$




$$
\begin{array}{r}
\left(D_{0}-\Delta_{A}\right) D_{k} D_{i} \Phi+i \Phi\left(i \Phi, D_{k} D_{i} \Phi\right)+\lambda \Phi\left(\Phi, D_{k} D_{i} \Phi\right)= \\
-2 i B D_{k}^{*} D_{i} \Phi-i \partial_{k}^{*} B D_{i} \Phi+2 i \partial_{k}^{*} B D_{i}^{*} \Phi \\
-2 i B D_{k} D_{i}^{*} \Phi+\frac{\lambda}{2} D_{k} D_{i} \Phi\left(1-|\Phi|^{2}\right) \\
-\lambda D_{i} \Phi\left(\Phi, D_{k} \Phi\right)-\lambda \Phi\left(D_{k} \Phi, D_{i} \Phi\right) \\
-i D_{k} \Phi\left(i \Phi, D_{i} \Phi\right)-i \Phi\left(i D_{k} \Phi, D_{i} \Phi\right) \\
-\lambda D_{k} \Phi\left(\Phi, D_{i} \Phi\right) .
\end{array}
$$

An important identity is for $w=\frac{1}{2}\left(1-|\Phi|^{2}\right)$, which satisfies

$$
\dot{w}-\Delta w+\lambda|\Phi|^{2} w=|D \Phi|^{2} .
$$

\section{A Priori Estimates.}

In this section we collect together the a priori estimates. In the first part we give energy, weighted energy and $L^{\infty}$ estimates. In the second part we give some estimates which are used to prove uniform convergence in the case $\lambda=1$. Throughout this section $\left(A_{0}, \Psi\right)=\left(A_{0}, A, \Phi\right)$ is a smooth solution to (2.1) so that there exists $r>0$ and $K=K\left(T_{l o c}\right)>0$ such that $\mathcal{U}=\left(B, D \Phi, \nabla B, D^{2} \Phi, w\right)$ satisfies

$$
|\mathcal{U}(x, t)| \leq K \exp \left(-\frac{r}{2}|x|\right) .
$$

for $t \leq T_{l o c}$ and $x \in \mathbb{R}^{2}$. This assumption is justified by the existence theorem 4.1. We shall also assume that $|\Phi(x, 0)| \leq 1$; theorem 3.3 will then ensure $|\Phi(x, t)| \leq 1$. We shall always state what gauge condition we are using in our estimates. However, by a slight notational abuse we shall always write the solution as $\left(A_{0}, A, \Phi\right)$ in any gauge. It is convenient to derive the estimates in the temporal gauge and then deduce from these the general case.

\subsection{Energy, Uniform Estimates and Exponential Decay.}

The following results describe a priori estimates useful to obtain existence of a smooth global solution. We start with energy identities which rely on the locally in time uniform exponential decay assumed above. These are the estimates of decreasing energy and a weighted energy estimate given respectively in the next two theorems. Notice that the estimates for the gauge invariant quantities $B,|D \Phi|, w$ implied by the energy estimates are 
valid in any gauge. In the following calculations all integrations by parts are justified by the exponential decay which we assume in (3.1).

Theorem 3.1 (Energy Identity). In temporal gauge

$$
\int_{0}^{T} \int_{\mathbb{R}^{2}}\left(|\dot{A}|^{2}+|\dot{\Phi}|^{2}\right) d x d t+\mathcal{E}(T)=\mathcal{E}(0)
$$

and in gauge invariant form,

$$
\int_{0}^{T} \int_{\mathbb{R}^{2}}\left(\left|\dot{A}-\nabla A_{0}\right|^{2}+\left|D_{0} \Phi\right|^{2}\right) d x d t+\mathcal{E}(T)=\mathcal{E}(0)
$$

where

$$
\mathcal{E}(t)=\int_{\mathbb{R}^{2}}\left(B(x, t)^{2}+|D \Phi(x, t)|^{2}+\frac{\lambda}{4}\left(1-|\Phi(x, t)|^{2}\right)^{2}\right) d x .
$$

Proof. This is a straightforward estimate obtained by multiplying the equations by $\dot{\Phi}$ and $\dot{A}$ respectively and integrating by parts (this is justified by the decay properties (3.1) which also guarantee the uniform integrability required to differentiate with respect to time under the integral sign). This gives (3.2) for as long as the solution exists that is, up to $T \leq T_{l o c}$. The gauge invariant form follows by a gauge transformation.

We now prove a weighted energy identity which is a generalization of an identity in [1].

Theorem 3.2 (Weighted Energy Identity). In temporal gauge

$\int_{0}^{T} \int_{\mathbb{R}^{2}}\left(\frac{r^{2}}{2}\left(|\dot{A}|^{2}+|\dot{\Phi}|^{2}\right)+\frac{\lambda}{4}\left(1-|\Phi|^{2}\right)^{2}-B^{2}\right) d x d t+\int_{\mathbb{R}^{2}} \frac{r^{2}}{2} e(T) d x=\int_{\mathbb{R}^{2}} \frac{r^{2}}{2} e(0) d x$ or in gauge invariant form

$$
\begin{aligned}
\int_{0}^{T} \int_{\mathbb{R}^{2}}\left(\frac{r^{2}}{2}\left(\left|\dot{A}-\nabla A_{0}\right|^{2}+\left|D_{0} \Phi\right|^{2}\right)+\frac{\lambda}{4}\left(1-|\Phi|^{2}\right)^{2}-B^{2}\right) d x d t & +\int_{\mathbb{R}^{2}} \frac{r^{2}}{2} e(T) d x \\
& =\int_{\mathbb{R}^{2}} \frac{r^{2}}{2} e(0) d x
\end{aligned}
$$

where $e$ is the energy density $e(t) \equiv e(A(t), \Phi(t))$ with $e(A, \Phi) \equiv$ $\frac{1}{2}\left(B^{2}+|D \Phi|^{2}+\frac{\lambda}{4}\left(1-|\Phi|^{2}\right)^{2}\right)$. (In the above integrands $r=|x|$.) 
Proof. First we obtain a Pohozaev type estimate: multiply the first equation in (2.5) by $x \cdot D \Phi$ (where $D=d-i A$ ) and integrate. We have

$$
\int_{\mathbb{R}^{2}}\left(x \cdot D \Phi, \partial_{t} \Phi\right)-\left(x \cdot D \Phi, \Delta_{A} \Phi\right)-\frac{\lambda}{2}(x \cdot D \Phi, \Phi)\left(1-|\Phi|^{2}\right) d x=0 .
$$

Upon integration the second term above gives (using the summation convention on repeated indices)

$$
\begin{aligned}
& \int_{\mathbb{R}^{2}}\left(x \cdot D \Phi, \Delta_{A} \Phi\right) d x= \\
& \int_{\mathbb{R}^{2}}\left(-|D \Phi|^{2}-\left(x_{i} D_{i} D_{j} \Phi, D_{j} \Phi\right)-\left(x_{i}\left[D_{j}, D_{i}\right] \Phi, D_{j} \Phi\right)\right) d x \\
& \quad=\int_{\mathbb{R}^{2}}\left(-|D \Phi|^{2}-\frac{1}{2}(x \cdot \nabla)|D \Phi|^{2}-\left(x_{i}\left[D_{j}, D_{i}\right] \Phi, D_{j} \Phi\right)\right) d x \\
& \quad=\int_{\mathbb{R}^{2}}\left(-\left(x_{i}\left[D_{j}, D_{i}\right] \Phi, D_{j} \Phi\right)\right) d x
\end{aligned}
$$

The third term becomes,

$$
\begin{aligned}
\int_{\mathbb{R}^{2}} \frac{\lambda}{2}(x \cdot D \Phi, \Phi)\left(1-|\Phi|^{2}\right) d x & =-\int_{\mathbb{R}^{2}} \frac{\lambda}{8}(x \cdot \nabla)\left(1-|\Phi|^{2}\right)^{2} d x \\
& =\int_{\mathbb{R}^{2}} \frac{\lambda}{4}\left(1-|\Phi|^{2}\right)^{2} d x
\end{aligned}
$$

Upon substituting these two into the integral above we obtain

$$
\int_{\mathbb{R}^{2}}\left(x \cdot D \Phi, \partial_{t} \Phi\right)+\left(i x_{i} B_{i j} \Phi, D_{j} \Phi\right)-\frac{\lambda}{4}\left(1-|\Phi|^{2}\right)^{2} d x=0
$$

where

$$
-i B_{i j}=\left[D_{i}, D_{j}\right]=-i\left(\partial_{i} A_{j}-\partial_{j} A_{i}\right)=-i B \sigma_{i j}
$$

with $\sigma_{i j}$ the antisymmetric tensor with $\sigma_{12}=+1$. We will use this below.

Next multiply the first equation in (2.5) by $r^{2} \partial_{t} \Phi$ and integrate to obtain

$$
\int_{\mathbb{R}^{2}} \frac{r^{2}}{2}\left(\left|\partial_{t} \Phi\right|^{2}-\left(\partial_{t} \Phi, \Delta_{A} \Phi\right)+\frac{\lambda}{8} \partial_{t}\left(1-|\Phi|^{2}\right)^{2}\right) d x=0 .
$$

We concentrate on the second term of (3.5): upon integrating by parts it becomes

$$
-\int_{\mathbb{R}^{2}} \frac{r^{2}}{2}\left(\partial_{t} \Phi, \Delta_{A} \Phi\right) d x=\int_{\mathbb{R}^{2}}\left(\left(x \cdot D \Phi, \partial_{t} \Phi\right)+\frac{r^{2}}{4} \partial_{t}|D \Phi|^{2}+\frac{r^{2}}{2}\left(D \Phi,\left[D, \partial_{t}\right] \Phi\right)\right) d x
$$


(and by substituting from (3.4))

$=\int_{\mathbb{R}^{2}}\left(-x_{i} B_{i j}\left(i \Phi, D_{j} \Phi\right)+\frac{\lambda}{4}\left(1-|\Phi|^{2}\right)^{2}+\frac{r^{2}}{4} \partial_{t}|D \Phi|^{2}+\frac{r^{2}}{2} \partial_{t} A \cdot(D \Phi, i \Phi)\right) d x$

$=\int_{\mathbb{R}^{2}}-x^{\prime} B \wedge(D \Phi, i \Phi)+* \frac{r^{2}}{2} \partial_{t} A \cdot(D \Phi, i \Phi)+\int_{\mathbb{R}^{2}}\left(\frac{\lambda}{4}\left(1-|\Phi|^{2}\right)^{2}+\frac{r^{2}}{4} \partial_{t}|D \Phi|^{2}\right) d x$

where we use (3.4), $\left[D, \partial_{t}\right] \Phi=i\left(\partial_{t} A\right) \Phi$ and $x^{\prime}=x_{i} d x_{i}$.

From the second equation of (2.5) we have, after multiplying by $\frac{r^{2}}{2} \partial_{t} A$

$$
\begin{aligned}
\int_{\mathbb{R}^{2}} \frac{r^{2}}{2}\left(\partial_{t} A,(D \Phi, i \Phi)\right) d x & =\int_{\mathbb{R}^{2}} \frac{r^{2}}{2}\left(\left|\partial_{t} A\right|^{2}+\left(\partial_{t} A, d^{*} d A\right)\right) d x \\
& =\int_{\mathbb{R}^{2}}\left(\frac{r^{2}}{2}\left|\partial_{t} A\right|^{2}+\frac{r^{2}}{4} \partial_{t} B^{2}\right) d x-\int_{\mathbb{R}^{2}}\left(x^{\prime} \wedge \partial_{t} A\right) B
\end{aligned}
$$

Also using the same equation

$$
\begin{aligned}
\int_{\mathbb{R}^{2}} x^{\prime} B \wedge(D \Phi, i \Phi) & =-\int_{\mathbb{R}^{2}} x^{\prime} B \wedge \partial_{t} A+\left(x^{\prime} \wedge * d B\right) B \\
& =-\int_{\mathbb{R}^{2}} x^{\prime} B \wedge \partial_{t} A+\frac{(x \cdot \nabla) B^{2}}{2} d x^{1} \wedge d x^{2}
\end{aligned}
$$

Taking the difference of the last equalities and substituting in (3.6) and then in (3.5) we obtain the weighted energy identity of theorem 3.2. The gauge invariant form follows by a gauge transformation.

The following three theorems are consequences of the maximum principle (theorem A.4). They state that $w$ globally controls the growth of $B$ and $D \Phi$. This will later be used in the estimates in the time asymptotics section 3.b (theorem 3.11) to infer the exponential decay of these quantities from the exponential decay of $w$. These results are analogues of results in [19].

Theorem 3.3. For all $\lambda>0$, if $|\Phi(x, 0)| \leq 1 \forall x$ then $|\Phi(x, t)| \leq 1 \forall x$. If in addition $\left|\Phi\left(x_{*}, 0\right)\right|<1$ for some $x_{*} \in \mathbb{R}^{2}$, then $|\Phi|<1$ on $\mathbb{R}^{2} \times(0, \infty)$. Equivalently, if $w(x, 0) \geq 0$ then $w \geq 0$ everywhere and if also $w\left(x_{*}, 0\right)>0$ at some point, then $w(\cdot, t)>0$ for $t>0$. 
Proof. Let $w=\frac{1}{2}\left(1-|\Phi|^{2}\right) \geq 0$. By $(2.16)$

$$
\dot{w}-\Delta w+\lambda(1-2 w) w \geq 0 \text { on } \mathbb{R}^{2} \times \mathbb{R}^{+} .
$$

Assuming that the initial data satisfy $w(x, 0)>0$ the maximum principle (theorem A.4) on (3.7) implies $w>0$ on $\mathbb{R}^{2} \times \mathbb{R}^{+}$.

Theorem 3.4. If $\lambda \leq 1$ and the initial data satisfy $|B(x, 0)| \leq w(x, 0)$ then for all $t>0,|B(x, t)| \leq w(x, t)$.

Proof. We note from (2.12) and (2.16) that

$$
\left(\partial_{t}-\Delta\right)(B-w)+|\Phi|^{2}(B-w)=-|\xi|^{2}+(\lambda-1)|\Phi|^{2} w \leq 0
$$

where $\xi \equiv \overline{\partial_{A}} \Phi=\frac{1}{2}\left(D_{1} \Phi+i D_{2} \Phi\right)$. A similar identity for $B+w$ completes the proof of the theorem.

Theorem 3.5. If $\lambda \leq 1$ and the initial data satisfy $|D \Phi(x, 0)| \leq 3 w(x, 0)$ and $|B(x, 0)| \leq w(x, 0)$ on $\mathbb{R}^{2}$ then

$$
|D \Phi(x, t)| \leq 3 w(x, t) \text { on } \mathbb{R}^{2} \times \mathbb{R}^{+} .
$$

Proof. Let $g=D \Phi$ and $g^{*}=D^{*} \Phi$. On $\{|g|=0\}$ the theorem clearly holds. Letting $V=\{|g|>0\}$ and applying equations (2.13), (2.16) and Kato's inequality,

$$
|g| \Delta|g| \geq\left(g, \Delta_{A} g\right)
$$

we have

$$
\begin{aligned}
|g|\left(\partial_{t}-\Delta\right)(|g|-3 w) \leq & |g| \partial_{t}|g|+\left(g, D_{0} g-2 i B g^{*}\right) \\
& -(g, i \Phi)^{2}-\lambda(g, \Phi)^{2} \\
& +(g, \lambda g w)-3|g|\left(|g|^{2}-\lambda|\Phi|^{2} w\right)
\end{aligned}
$$

We note

$$
\begin{gathered}
|g| \partial_{t}|g|-\left(g, D_{0} g\right)=0 \\
(g, i \Phi)^{2}+\lambda(g, \Phi)^{2}=(1-\lambda)(g, i \Phi)^{2}+\lambda|g|^{2}|\Phi|^{2} \geq 0 \quad \text { for } \lambda \leq 1 \\
-\left(g, i B g^{*}\right) \leq|g|^{2}|B| \leq|g|^{2} w
\end{gathered}
$$

using theorem 3.4. Inserting these in (3.8) we obtain

$$
|g|\left(\partial_{t}-\Delta\right)(|g|-3 w) \leq-|g|\left(\lambda|\Phi|^{2}|g| \lambda w|g|+|g|^{2}-3 \lambda|\Phi|^{2} w-2|g| w\right)
$$




$$
\begin{aligned}
& \leq-|g|\left(\lambda|\Phi|^{2}(|g|-3 w)+|g|(-\lambda w+|g|-2 w)\right) \\
& \leq-|g|\left(|g|+\lambda|\Phi|^{2}\right)(|g|-3 w)
\end{aligned}
$$

and thus

$$
\left(\partial_{t}-\Delta\right)(3 w-|g|)+\left(|g|+\lambda|\Phi|^{2}\right)(3 w-|g|) \geq 0 \quad \text { on } V .
$$

The result now follows from the maximum principle (theorem A.4).

We can now establish uniform estimates. We show that all the (covariant) derivatives of $B, D \Phi$ and bounded in $L^{\infty}\left(\mathbb{R}^{2}\right)$ independent of time $T_{\text {loc }}$ (and in particular independent of the time interval in which they are assumed to exist). Since the $L^{\infty}$ norms of these quantities are gauge invariant we may use the equations in the temporal gauge. The argument follows Hassel's ([18]) adaptation of Hamilton's ([16]) method.

Theorem 3.6 (Uniform Estimates and Uniform Continuity). $\Psi$ satisfies estimates of the form

$$
\begin{aligned}
& \sup _{t>0} \sup _{x \in \mathbb{R}^{2}}\left(\left|\partial^{\alpha} B(x, t)\right|+\left|D^{\alpha} D \Phi(x, t)\right|\right) \leq \\
& \quad c\left(|\alpha|, \mathcal{E}(0),\left|\partial^{\alpha} B(x, 0)\right|_{L^{\infty}\left(\mathbb{R}^{2}\right)},\left|D^{\alpha} D \Phi(x, 0)\right|_{L^{\infty}\left(\mathbb{R}^{2}\right)}\right)
\end{aligned}
$$

for each multi-index $\alpha$. Therefore, $\partial^{\alpha} B(x, t)$ and $\left|D^{\alpha} \Phi(x, t)\right|^{2}$ are uniformly continuous on $\mathbb{R}^{2} \times \mathbb{R}^{+}$and from (2.1) the quantities $\partial_{t} A-\nabla A_{0}$ and $\left|D_{0} \Phi\right|^{2}$ are uniformly continuous on $\mathbb{R}^{2} \times \mathbb{R}^{+}$. (These estimates hold throughout the time of existence).

The method of proof uses the lemma below. We let $\zeta=(B, D \Phi)$ and differentiate with the direct sum connection i.e., $D_{i} \zeta=\left(\partial_{i} B, D_{i} D \Phi\right)$ etc. The following differential inequalities follow from (2.12)-(2.15):

$$
\left(\partial_{t}-\Delta\right)|\zeta|^{2}+2 \sigma|\Phi|^{2}|\zeta|^{2} \leq C_{1}|\zeta|^{3}+\lambda w|\zeta|^{2}-2|D \zeta|^{2}
$$

$$
\left(\partial_{t}-\Delta\right)|D \zeta|^{2}+2 \sigma|\Phi|^{2}|D \zeta|^{2} \leq C_{2}\left(|\zeta|^{2}|D \zeta|+|\zeta||D \zeta|^{2}\right)+\lambda w|D \zeta|^{2}
$$

where $\sigma=\min (1, \lambda)$ and $C_{1}, C_{2}$ are constants. 
Lemma 3.7. Consider a smooth function $\zeta$ on $\mathbb{R}^{2} \times \mathbb{R}^{+}$which satisfies (3.11)-(3.12) and

$$
\sup _{T_{*}-1 / K \leq t \leq T_{*}, x \in \mathbb{R}^{2}}|\zeta(x, t)| \leq K
$$

and assume $K \geq 1$ and $T_{*}>K^{-1}$. Then there exists a number $C_{3}$ such that

$$
\sup _{\left(T_{*}-1 / 2 K\right) \leq t \leq T_{*}} \sup _{x \in \mathbb{R}^{2}}|D \zeta(x, t)| \leq C_{3} K^{3 / 2} .
$$

Proof. Let $\delta=\frac{1}{K}$, so $T_{*}>\delta$ and consider the function

$$
Q \equiv\left(t-T_{*}+\delta\right)\left(|D \zeta|^{2}-b K^{3}\right)+a|\zeta|^{2}
$$

on $\left[T_{*}-\delta, T_{*}\right] \times \mathbb{R}^{2}$, then we find

$$
\begin{aligned}
\dot{Q}-\Delta Q \leq & |D \zeta|^{2}+a\left(C_{1}|\zeta|^{3}+\lambda w|\zeta|^{2}-2|D \zeta|^{2}\right)-b K^{3} \\
& +\left(t-T_{*}+\delta\right)\left(C_{2}\left(|\zeta|^{2}|D \zeta|+|\zeta||D \zeta|^{2}\right)+\lambda w|D \zeta|^{2}\right) \\
\leq \quad(1- & \left.2 a+2\left(t-T_{*}+\delta\right) c K+\lambda\right)|D \zeta|^{2} \\
& +\left(a c+\lambda a+\left(t-T_{*}+\delta\right) c-b\right) K^{3} .
\end{aligned}
$$

Since $\left(t-T_{*}+\delta\right) \leq \delta=\frac{1}{K}$ we see that for $a$ and $b$ sufficiently large (independent of $K$ )

$$
\dot{Q}-\Delta Q \leq 0
$$

We now apply the maximum principle on $\left[T_{*}-\delta, T_{*}\right] \times \mathbb{R}^{2}$ to deduce

$$
\left(t-T_{*}+\delta\right)\left(|D \zeta|^{2}-b K^{3}\right)+a|\zeta|^{2} \leq a K^{2}
$$

It follows from this that

$$
|D \zeta(x, t)| \leq \frac{c K}{\left(t-T_{*}+\delta\right)^{1 / 2}}+\sqrt{b} K^{3 / 2}
$$

which gives the result by letting $t \in\left[T_{*}-\delta / 2, T_{*}\right]$.

From this and the energy estimates we can infer the uniform estimates:

Proof of theorem 3.6. The strategy is to assume that at some time, $T_{*},|\zeta|_{L^{\infty}}$ has magnitude $K$ and use the previous lemma to show this leads to a contradiction of the energy estimate $\mathcal{E}\left(T_{*}\right) \leq \mathcal{E}(0)$ for sufficiently large 
$K$. The next claim shows that by taking $K$ large we can indeed apply the previous lemma.

Claim. Let $u$ be a positive solution of the differential inequality on $\mathbb{R}^{2} \times \mathbb{R}^{+}$

$$
\begin{aligned}
& \frac{d}{d t} u^{2}-\Delta u^{2} \leq a u^{2}+b u^{3} \\
& u(x, 0)=u_{0}(x)
\end{aligned}
$$

with $a, b>0$, and satisfying

$$
\lim _{|x| \rightarrow \infty}|u(x, t)|=0
$$

for all $t$. Define $\mu \equiv \sqrt{1+\left|u_{0}\right|_{\infty}^{2}}$ and let $K>\max \{2 \mu,(a+b) \mu\}$. Then for $t<T_{c}=\frac{1}{(a+b) \mu}>\frac{1}{K}$ we have

$$
|u| \leq K \text {. }
$$

Proof of Claim. Compare $u^{2}$ with the solution to the ODE

$$
\begin{aligned}
\dot{f} & =(a+b) f^{3 / 2} \\
f(0) & =1+\left|u_{0}\right|_{L^{\infty}\left(\mathbb{R}^{2}\right)}^{2}
\end{aligned}
$$

Noting that $f>1$ we have

$$
\frac{d}{d t}\left(u^{2}-f\right)-\Delta u^{2} \leq a u^{2}+b u^{3}-(a+b) f^{\frac{3}{2}}
$$

and conclude that $u^{2}<f$ (or else, let $t_{0}$ be the first time so that $\left(u^{2}-\right.$ $f)\left(x_{0}, t_{0}\right)=0$; at this point the inequality above is contradicted). Thus

$$
u^{2}<\frac{4 \mu^{2}}{(2-(a+b) \mu t)^{2}}
$$

and so for $t \leq T_{c}=\frac{1}{(a+b) \mu}$ it follows that $|u| \leq 2 \mu \leq K$ which proves the claim.

Apply the above claim with $|u|=3$ to conclude the theorem up to time $T_{c}$. We now apply lemma 3.7 to conclude the theorem for times in $\left[T_{c}, T_{l o c}\right]$ : we assume that at some time $T_{*}$ we have $\sup _{x}\left|\zeta\left(x, T_{*}\right)\right|=K$ and obtain a contradiction for large $K$. By the previous claim we may assume $T_{*}>\frac{1}{K}$ by making $K$ large compared with $|\zeta(0)|_{L^{\infty}\left(\mathbb{R}^{2}\right)}$. Therefore by the previous lemma we know that for some $c>0$

$$
\sup _{x}\left|D \zeta\left(x, T_{*}\right)\right| \leq c K^{3 / 2}
$$


Since $\lim _{|x| \rightarrow \infty}\left|\zeta\left(x, T_{*}\right)\right|=0$ there is a point $x_{*}$ at which

$$
\left|\zeta\left(x_{*}, T_{*}\right)\right|=K
$$

so that there exists a number $\rho$ such that

$$
\left|\zeta\left(x, T_{*}\right)\right| \geq \frac{K}{2} \text { for }\left|x-x_{*}\right| \leq \frac{\rho}{\sqrt{K}} .
$$

From this we obtain a lower bound for the energy

$$
\begin{aligned}
\mathcal{E}\left(T_{*}\right) & \geq \int|\zeta|^{2} \geq \frac{1}{4} K^{2} \times\left(\frac{\pi \rho^{2}}{K}\right) \\
& \geq \frac{\pi \rho^{2} K}{4}
\end{aligned}
$$

which gives a contradiction to $\mathcal{E}\left(T_{*}\right) \leq \mathcal{E}(0)$ for $K$ large compared with the energy and satisfying the condition in the previous claim. This completes the proof of the theorem for the case of no derivatives. The proof of the uniform bounds for the higher derivatives is exactly as in [18]. To prove that $|D \zeta|$ is bounded notice that (3.11)-(3.12) imply that if $A$ is large enough then

$$
\left(\partial_{t}-\Delta+1\right)\left(A|\zeta|^{2}+|D \zeta|^{2}\right) \leq c(1+1|\zeta|)^{4} .
$$

From this the maximum principle implies that if $|\zeta(x, t)|_{L^{\infty}\left(\mathbb{R}^{2}\right)}$ is bounded independent of time then so is $|D \zeta(x, t)|_{L^{\infty}\left(\mathbb{R}^{2}\right)}$. This argument can be repeated indefinitely to complete the proof of the theorem.

\subsection{Estimates for Time Asymptotics.}

The results in this section will be used to describe the behaviour of the solution as time goes to infinity. Time independent $L^{p}$ estimates will be valid for all values of $\lambda$ while the results of exponential decay following will hold for restricted values of $\lambda$. As mentioned above, the main asymptotic result showing that the solution converges strongly to a static solution holds only in the case of $\lambda=1$. To begin, the next theorem gives a condition under which vortices cannot escape to infinity. This will later be crucial in proving the existence of a uniform limit of the solution in the case of $\lambda=1$.

Theorem 3.8. Let $\lambda=1$ and assume the initial magnetic field satisfies the condition $|B(x, 0)| \leq w(x, 0)$. Then the weighted energy defined in theorem 
3.2 is bounded independent of time:

$$
\int_{\mathbb{R}^{2}} r^{2} e(t) d x \leq c<\infty .
$$

Moreover, for all positive $\epsilon$ there exists a number $R(\epsilon)$, independent of time, such that for all $t>0$

$$
|x|>R(\epsilon) \Rightarrow|w(x, t)|+|B(x, t)|+|D \Phi(x, t)|<\epsilon .
$$

Proof. The first statement follows immediately from the fact (theorem 3.4) that $|B| \leq w$ and theorem 3.2. To deduce the second statement from this, assume to the contrary that there exists $\epsilon_{*}>0$ and a sequence of points in space-time $\left(x_{i}, t_{i}\right)$ with $t_{i}>0$ and $\left|x_{i}\right| \nearrow+\infty$ such that

$$
\left|w\left(x_{i}, t_{i}\right)\right| \geq \epsilon_{*} .
$$

Then by the uniform continuity of $w$ there exist balls $B\left(x_{i} ; \rho\right)$ on which $|w| \geq$ $\frac{\epsilon_{*}}{2}$, which gives an immediate contradiction to the uniform boundedness of the weighted energy. The same argument applies to $B$ and $D \Phi$.

We continue with time independent estimates in $L^{1}$ for the gauge invariant quantities $B,|D \Phi|, w$ using the temporal gauge.

Theorem 3.9 ( $L^{1}$ Estimates Uniform in Time). Assume $\lambda>0$. Then there exists

$$
c=c\left(\|w(0)\|_{L^{1}},\left\|D^{\alpha_{1}} \Phi(0)\right\|_{L^{1} \cap L^{\infty}},\left\|\nabla^{\alpha_{2}} B(0)\right\|_{L^{1} \cap L^{\infty}}, \mathcal{E}(0)\right)
$$

for $\left|\alpha_{1}\right| \leq 2$ and $\left|\alpha_{2}\right| \leq 1$ such that

$$
\begin{aligned}
\sup _{t>0}\left(\|B(t)\|_{L^{1}\left(\mathbb{R}^{2}\right)}+\|w(t)\|_{L^{1}\left(\mathbb{R}^{2}\right)}+\|D \Phi(t)\|_{L^{1}\left(\mathbb{R}^{2}\right)}\right. & +\left\|D^{2} \Phi(t)\right\|_{L^{1}\left(\mathbb{R}^{2}\right)} \\
+ & \left.\|\nabla B(t)\|_{L^{1}\left(\mathbb{R}^{2}\right)}\right) \leq c .
\end{aligned}
$$

Proof. To prove this we consider $\beta(x, t)=e^{t} B(x, t)$ and observe that it satisfies the equation

$$
\begin{aligned}
\dot{\beta}-\Delta \beta & =e^{t}\left(B\left(1-|\Phi|^{2}\right)+2\left(i D_{1} \Phi, D_{2} \Phi\right)\right) \\
& \equiv e^{t} h(x, t)
\end{aligned}
$$


where it follows from the energy estimate that $\sup _{t>0}\|h(t)\|_{L^{1}\left(\mathbb{R}^{2}\right)}<c<\infty$, with $c$ depending only on $\mathcal{E}(0)$. Let $K_{t}$ be the heat kernel and write the solution as

$$
\beta(t)=\int_{0}^{t} K_{t-s} * h(s) e^{s} d s+K_{t} * \beta(0)
$$

where $*$ means spatial convolution. This leads to the estimate

$$
\begin{aligned}
\|\beta(t)\|_{L^{1}\left(\mathbb{R}^{2}\right)} & \leq\|\beta(0)\|_{L^{1}\left(\mathbb{R}^{2}\right)}+\int_{0}^{t}\|h(s)\|_{L^{1}\left(\mathbb{R}^{2}\right)} e^{s} d s \\
& \leq c\left(e^{t}-1\right)+\|\beta(0)\|_{L^{1}\left(\mathbb{R}^{2}\right)}
\end{aligned}
$$

which implies the following estimate for $B$ :

$$
\|B(t)\|_{L^{1}\left(\mathbb{R}^{2}\right)} \leq e^{-t}\|B(0)\|_{L^{1}\left(\mathbb{R}^{2}\right)}+c\left(1-e^{-t}\right) .
$$

The proof of the other results is very similar so we will be very brief. An identical argument applied to equation (2.16) gives the estimate for $w$. Next integrate (3.12) over $\mathbb{R}^{2}$ and estimate for each $t \geq 0$

$$
\int|\zeta \| D \zeta|^{2} \leq \epsilon \int|D \zeta|^{2}+\frac{4}{\epsilon} \int|\zeta|^{2}|D \zeta|^{2}
$$

This together with the known estimates for $\|D \zeta\|_{L^{\infty}},\|\zeta\|_{L^{2}}$ and $\|w\|_{L^{1}}$ gives the inequality

$$
\partial_{t} \int|D \zeta|^{2}+2 \int|D \zeta|^{2} \leq c
$$

from which follows the $L^{2}$ boundedness of $\nabla B, D_{A}^{2} \Phi$ independent of time. Next substitute this information into (2.14) and apply the argument above to deduce the $L^{1}$ boundedness of $\nabla B$ independent of time. Next write down equations for $\left(\Phi, D_{A} \Phi\right),\left(i \Phi, D_{A} \Phi\right),\left(\Phi, D_{A}^{2} \Phi\right),\left(i \Phi, D_{A}^{2} \Phi\right)$ by taking the inner product of (2.13) and (2.15) with $\Phi$ and $i \Phi$. Applying the same argument to these equations will complete the proof.

We now restrict to the Coulomb gauge and obtain estimates for the connection by solving the elliptic system

$$
\begin{aligned}
& \partial_{1} A_{1}+\partial_{2} A_{2}=0 \\
& \partial_{1} A_{2}-\partial_{2} A_{1}=B .
\end{aligned}
$$

The solution to this system is unique only up to gauge transformation by a harmonic function. Thus there is a unique solution for $A$ which has limit zero as $|x| \rightarrow \infty$. It is this solution which we investigate. 
Theorem 3.10 (Uniform $L^{p}$ estimates). Let $\lambda>0$. In the Coulomb gauge (2.7) the connection $A(t)$ satisfies the time independent estimates

$$
\|A(t)\|_{L^{p}\left(\mathbb{R}^{2}\right)} \leq c(p)\left(\|B(t)\|_{L^{1}\left(\mathbb{R}^{2}\right)}+\|B(t)\|_{L^{\infty}\left(\mathbb{R}^{2}\right)}\right)
$$

for all $2<p \leq \infty$. There exist constants $c=c(|\alpha|)$, independent of time, such that for each multi-index $\alpha$

$$
\sup _{x}\left(\left|\nabla_{x}^{\alpha} A(x, t)\right|+\left|\partial_{x}^{\alpha} \Phi(x, t)\right|\right) \leq c(|\alpha|) .
$$

Proof. The solution of the elliptic system above is

$$
A_{i}(x, t)=\frac{\sigma_{i j}}{2 \pi} \int_{\mathbb{R}^{2}} \frac{x_{j}-y_{j}}{|x-y|^{2}} B(y, t) d y
$$

where $\sigma$ is the antisymmetric tensor and $\sigma_{12}=+1$. This is of the form $A=$ $K * B$, where the kernel $K$ is in the weak $L^{2}$ space. The estimates for $p<\infty$ then follow from the convolution estimates (see for example [14, p. 232]) and the known estimates for $B$. The $L^{\infty}$ estimate is obtained by splitting the integral into $|x-y| \leq 1$ and $|x-y| \geq 1$. To prove the boundedness of all derivatives of $A$ introduce a mollifying function $\rho(x) \in C_{0}^{\infty}\left(\mathbb{R}^{2}\right)$, nonnegative, equal to 1 for $|x| \leq 1$ and equal to 0 for $|x| \geq 2$. We can then write $A=A^{1}+A^{2}$ where

$$
\begin{aligned}
& A_{i}^{1}(x, t)=\frac{\sigma_{i j}}{2 \pi} \int_{\mathbb{R}^{2}} \frac{x_{j}-y_{j}}{|x-y|^{2}} \rho(x-y) B(y, t) d y \\
& \quad=\frac{\sigma_{i j}}{2 \pi} \int_{\mathbb{R}^{2}} \frac{u_{j}}{|u|^{2}} \rho(u) B(x-u, t) d u \\
& A_{i}^{2}=\frac{\sigma_{i j}}{2 \pi} \int_{\mathbb{R}^{2}} \frac{x_{j}-y_{j}}{|x-y|^{2}}(1-\rho(x-y)) B(y, t) d y .
\end{aligned}
$$

The boundeness of $B$ in $L^{1}$ and the uniform boundedness of all derivatives of $B$ imply the uniform bound of all derivatives of $A$. The estimates for $\Phi$ then follow since $D_{A} \Phi=\nabla \Phi-i A \Phi$ and all the covariant derivatives are bounded in $L^{\infty}$.

We now restrict to the case of $\lambda \leq 1$ and show that the quantities $w, B, D \Phi, D^{2} \Phi, \nabla B$ decay exponentially as $|x| \rightarrow \infty$. In the case of $\lambda=1$ this decay is uniform in time. This fact depends crucially on theorem 3.8. Recall that $w=\frac{1}{2}\left(1-|\Phi|^{2}\right)$ and $0<w \leq \frac{1}{2}$. 
Theorem 3.11 (Exponential Decay). Let $\lambda \leq 1$. Assume that for any $\epsilon \in(0,1)$ there is a $C(\epsilon)$ such that $\left(w+\left|D_{k} D_{i} \Phi\right|+|\nabla B|\right)(x, 0)<$ $C(\epsilon) e^{-\sqrt{\lambda}(1-\epsilon)|x|}$ on $\mathbb{R}^{2}$ and that $(w, B, D \Phi)(x, 0)$ satisfy the hypotheses in theorems 3.4 and 3.5. Then for any $\epsilon \in(0,1)$ there is a constant $M(\epsilon, T)$ such that

$\left(w+|B|+|D \Phi|+\left|D^{2} \Phi\right|+|\nabla B|\right)(x, t)<M(\epsilon, T) e^{-\sqrt{\lambda}(1-\epsilon)|x|}$ on $\mathbb{R}^{2} \times[0, T]$.

When $\lambda=1$ then $M=M(\epsilon)$ and (3.17) holds on $\mathbb{R}^{2} \times \mathbb{R}^{+}$and implies exponential decay uniformly in time, that is,

$$
\left(w+|B|+|D \Phi|+\left|D^{2} \Phi\right|+|\nabla B|\right)(x, t)<M(\epsilon) e^{-\sqrt{\lambda}(1-\epsilon)|x|} \text { on } \mathbb{R}^{2} \times \mathbb{R}^{+} .
$$

Proof. Let $m=m(\epsilon)=\sqrt{\lambda}(1-\epsilon)$ and find $R=R(\epsilon, T)$ such that $0<w<$ $\frac{\lambda \epsilon(2-\epsilon)}{9+2 \lambda}$ on $\{|x| \geq R, t \leq T\}$ and set

$$
v(x)=M_{1}(\epsilon) e^{m(R-|x|)} .
$$

Then

$$
\Delta v=\left(\frac{-m}{|x|}+m^{2}\right) v \leq m^{2} v .
$$

By (2.16) and since $|g| \leq 3 w$ (cf. theorem 3.5) we have

$$
\begin{aligned}
\left(\partial_{t}-\Delta\right)(v-w) & \geq-m^{2} v-9 w^{2}+\lambda(1-2 w) w \\
& \geq-m^{2}(v-w)
\end{aligned}
$$

on $\{|x| \geq R, t \leq T\}$. Next choose $M_{1}>\max \{1, C(\epsilon)\}$. Notice that $v-w>0$ on $\{|x|=R\} \bigcup\{t=0\}$ so the maximum principle (theorem A.4) implies that $v-w \geq 0$ for $|x| \geq R$ and thus (3.17) follows with $M=M_{1} e^{m R}$.

When $\lambda=1$, then $w \rightarrow 0$ as $|x| \rightarrow \infty$ uniformly in time by theorem 3.8 , so $M_{1}, R$ are independent of time and so $M(\epsilon, T)=M(\epsilon)$ and (3.17) is valid on $\mathbb{R}^{2} \times \mathbb{R}^{+}$. Since $|B| \leq w$ and $\left|D_{A} \Phi\right| \leq 3 w$ when $\lambda \leq 1$ these quantities decay as $w$ does.

Next we let $h$ be the matrix $D^{2} \Phi$ with $h_{k i}=D_{k} D_{i} \Phi$ and consider equation (2.15) and Kato's inequality ([19])

$$
|h| \Delta|h| \geq\left(h, \Delta_{A} h\right) .
$$

It follows that on the set $V=\{|h|>0\}$,

$$
|h|\left(\partial_{t}-\Delta\right)|h| \leq|h| \partial_{t}|h|-\left(h, \Delta_{A} h\right)=\left(h, \dot{h}-\Delta_{A} h\right)
$$




$$
\begin{array}{cc}
\leq & -\lambda|\Phi|^{2}|h|^{2}-(1-\lambda)|(i \Phi, h)|^{2}+|h|^{2}(4|B|+\lambda w) \\
& +|h|\left(3|\nabla B \| g|+(3 \lambda+2)|\Phi||g|^{2}\right) \\
& \leq \quad-\lambda|\Phi|^{2}|h|^{2}+a(x, t)|h|^{2}+b(x, t)|h|
\end{array}
$$

where $a(x, t), b(x, t)$ are positive and decay exponentially by the above. Indeed since $|\nabla B|$ is bounded independent of time, for each $\epsilon>0$ there exists $c_{1}(\epsilon)$ such that

$$
b(x, t) \leq c_{1}(\epsilon) e^{-(1-\epsilon)|x|}
$$

and there is $R(\epsilon)$, independent of time, such that

$$
a(x, t)<\epsilon, \quad b(x, t)<\epsilon, \quad|\Phi|>\frac{1}{2} \quad \forall t>0, \quad|x|>R(\epsilon) .
$$

Thus there is $n(\epsilon)>0$ satisfying

$$
\lim _{\epsilon \rightarrow 0} n(\epsilon)=0
$$

such that on $V \cap\{|x|>R(\epsilon)\}$,

$$
\left(\partial_{t}-\Delta\right)|h|+(1-n(\epsilon))|h| \leq c_{2}(\epsilon) e^{-(1-\epsilon)|x|} .
$$

Introduce a comparison function

$$
v(x)=\Lambda(\epsilon) e^{-k(\epsilon)|x|}
$$

with

$$
\Lambda(\epsilon)>\max \left\{\frac{c_{2}(\epsilon)}{\epsilon k(\epsilon)^{2}}, L e^{k(\epsilon) R(\epsilon)}\right\}, \quad k(\epsilon)<\min \left\{(1-\epsilon), \sqrt{\frac{1-n(\epsilon)}{(1+\epsilon)}}\right\}
$$

where $L>\|h\|_{L^{\infty}\left(\mathbb{R}^{2} \times \mathbb{R}^{+}\right)}$. Notice that this is independent of time. Then $\Delta v \leq k(\epsilon)^{2} v$ and so together with (3.18) this implies

$$
\begin{aligned}
\left(\partial_{t}-\Delta\right)(v-|h|) \geq & -k(\epsilon)^{2} v+(1-n(\epsilon))|h|-c_{2}(\epsilon) e^{-(1-\epsilon)|x|} \\
& \geq-(1+\epsilon) k(\epsilon)^{2}(v-|h|)+\left(-(1+\epsilon) k(\epsilon)^{2}+(1-n(\epsilon))|h|\right. \\
& \geq-(1+\epsilon) k(\epsilon)^{2}(v-|h|) .
\end{aligned}
$$

Thus by the maximum principle as in theorem $3.5|h| \leq v$ and the estimate for $D^{2} \Phi$ follows. Using this and a similar comparison function we also infer the estimate for $\nabla B$. 


\section{Existence and Uniqueness.}

Using gauge equivalence it suffices to prove the existence and uniqueness of the solution of the equations in the uniformly parabolic gauge (2.10). General results imply local existence. From the a priori estimates derived in the previous section follows the existence of a unique global solution of the equations. Uniqueness of the fully invariant equations (2.1) is meant in the sense of a unique gauge equivalence class of solutions. In specific gauges the equations may or may not possess a single solution. In the three gauges under consideration, namely the uniformly parabolic, temporal and Coulomb gauges, there is a unique bounded smooth solution. (We restrict to bounded solutions which ensures solutions of (2.6) and (2.9) are unique.)

To obtain local existence we apply theorem A.1 stated in the appendix to (2.10) and we obtain a unique solution $\Psi=(A, \Phi)$ up to time $T_{l o c}$. Using this solution we then regard $(A, \Phi)$ as known and apply theorem A.2 to the initial value linear system of equations (2.12)-(2.16). Thus we obtain exponential decay in space for $\mathcal{U}=\left(B, D \Phi, \nabla B, D^{2} \Phi, w\right)$ for each time $t \leq T_{\text {loc }}$. In summary we have:

Theorem 4.1 (Local Existence). Assume that the initial data $(A, \Phi, B$, $\left.D \Phi, \nabla B, D^{2} \Phi, w\right)$ belong to the class $\mathcal{C}^{r, N}$ defined in the introduction. Then there exists a time $T_{l o c}$ (depending on the initial data) up to which an unique solution to equations (2.10) exists with each component in $C^{2,1, \frac{1}{2}}\left(\mathbb{R}^{2} \times\right.$ $\left.\left[0, T_{l o c}\right]\right)$. Moreover, there exists a constant $K=K\left(T_{l o c}\right)$ such that $\mathcal{U}=$ $\left(B, D \Phi, \nabla B, D^{2} \Phi, w\right)$ satisfies

$$
|\mathcal{U}(x, t)| \leq K \exp \left(-\frac{r}{2}|x|\right)
$$

for all $t \leq T_{\text {loc }}$ and $x \in \mathbb{R}^{2}$.

We may now conclude:

Theorem 4.2 (Global Existence and Uniqueness). Consider the initial value problem for (2.5) in temporal gauge with initial data in $\mathcal{C}^{r, N}$. There exists a unique global smooth solution $(A(t), \Phi(t))$ for $t>0$ which is bounded for each $t$, has winding number $N$ and satisfies the energy identities of theorems 3.1 and 3.2, and the $L^{\infty}$ and $L^{1}$ estimates of theorem 3.6 and theorem 3.9. Furthermore, the solution takes on the initial values in the sense that

$$
\lim _{t \rightarrow 0}\left(\|\Psi(t)-\Psi(0)\|_{L^{\infty}}+\|\mathcal{U}(t)-\mathcal{U}(0)\|_{L^{\infty}}\right)=0 .
$$


Proof. From theorem 4.1 it follows that in the uniformly parabolic gauge there exists a unique local solution in the space $C^{2,1, \frac{1}{2}}\left(\mathbb{R}^{2} \times\left[0, T_{l o c}\right]\right)$, where $T_{l o c}$ is some time of existence, and the solution satisfies the initial condition. As it also satisfies the estimates of theorem 3.6 we conclude from the second equation in (2.10) that $\dot{A}-\Delta A$ is uniformly bounded in $(x, t)$ and thus for each $t>0,\|A(t)\|_{L^{\infty}(d x)} \leq c(t+1)$. By differentiating the same equation we obtain similarly that $A$ and all its spatial derivatives have at most linear growth with respect to time. Recall that in the uniformly parabolic gauge $A_{0}=d^{*} A$, which together with its spatial derivatives is now uniformly bounded on any finite time interval, and apply the gauge transformation $\chi$ determined by (2.4), $\dot{\chi}=A_{0}=d^{*} A$ and $\chi(0)=0$, to move into temporal gauge. Hence $\chi$ and its spatial derivatives are $\leq c t$ (uniform in $x$ ) and, therefore, $\lim _{t \rightarrow 0}\left(|\chi(t)|_{L^{\infty}\left(\mathbb{R}^{2}\right)}+|d \chi(t)|_{L^{\infty}\left(\mathbb{R}^{2}\right)}\right)=0$. Thus the solution in the temporal gauge takes on the initial values as claimed. The fact that $\mathcal{U}$ takes on the initial values uniformly follows from theorem A.2. It then follows by integration of (2.5) with respect to time that $A, \Phi$ and their derivatives can grow at most linearly with respect to time. We can therefore continue the solution for all time to obtain a global smooth solution of the inital value problem, which attains the initial data. Finally the uniqueness of the solution in the temporal gauge follows from the uniqueness in the uniformly parabolic gauge and equations (2.4) and (2.9). Indeed, the gauge transformations determined by these equations are unique on account of the condition that the solution be bounded at each time which removes nonuniqueness of (2.9).

\section{Large Time Asymptotics.}

This section is divided into two parts. In the first we prove that for each $\lambda>0$ the solution $(A, \Phi)$ possesses a subsequence converging to a solution of the static equations in a weak topology of a suitable $L^{p}$ space and also in the compact-open topology of convergence of all derivatives (uniform convergence of all derivatives on compact sets of $\mathbb{R}^{2}$ ). This is done using the equations in Coulomb gauge. We then restrict further to the case of $\lambda=1$ and show that (after applying appropriate time-dependent gauge transformations) this (subsequential) convergence is strong in $H^{2}$. In the second section using an adiabatic approximation it is proved that once the solution is (strongly) close to the moduli space the entire sequence converges in a certain gauge. It is then finally shown that the sequence 
converges in the temporal gauge.

\subsection{Extraction of Convergent Subsequence.}

We will now show weak subsequential convergence to a solution of the static equations in the Coulomb gauge.

Theorem 5.1 (Weak Convergence). Consider the solution $(A(t), \Phi(t))$ given by theorem 4.2 in the Coulomb gauge and let $\lambda>0$. For any $2<p \leq$ $\infty$ there is a subsequence $t_{\nu} \nearrow+\infty$ and a weak limit $\left(A_{\infty}, \Phi_{\infty}\right)$ such that

$$
\begin{array}{ll}
A\left(t_{\nu}\right) \rightarrow A_{\infty} & \text { weakly in } L^{p} \\
& \text { weak* in } L^{\infty} \text { and in } C^{\infty}(K) \text { for all compact sets } K \\
\Phi\left(t_{\nu}\right) \rightarrow \Phi_{\infty} & \text { weak } * \text { in } L^{\infty} \text { and in } C^{\infty}(K) \text { for all compact sets } K
\end{array}
$$

The quantities $D_{0} \Phi, \dot{A}-\nabla A_{0}$ are uniformly continuous and

$$
\lim _{|x|+t \rightarrow \infty}\left(D_{0} \Phi, \dot{A}-\nabla A_{0}\right)=(0,0) .
$$

Thus the weak limit $\left(A_{\infty}, \Phi_{\infty}\right)$ is a weak solution of the static problem.

Proof. The statement of weak convergence follows from weak compactness of bounded sets in $L^{p}$ for $p \in(2, \infty)$ and theorem 3.10. Similarly, recall from theorem 3.3 that $|\Phi|(x, t) \leq 1$ and thus weak* compactness follows. The uniform estimates of theorems 3.6 and 3.10 and the Ascoli-Arzela theorem imply compactness in the compact-open topology in $C^{\infty}\left(\mathbb{R}^{2}\right)$. Since in the Coulomb gauge $A$ is uniformly bounded (c.f. theorem 3.10) then theorem 3.6 also implies the uniform continuity of $D^{\alpha} \Phi$ (and not only of $\left|D^{\alpha} \Phi\right|^{2}$ ) as well of $\partial^{\alpha} B$; hence by equations (2.1), the quantities $D_{0} \Phi, \dot{A}-\nabla A_{0}$ are uniformly continuous on $\mathbb{R}^{2} \times \mathbb{R}^{+}$. By the energy identity (3.3) we infer that (5.1) holds: for if not, then for some $\epsilon_{0}>0$ there is a sequence of points $P_{i}=\left(x_{i}, t_{i}\right)$ and balls $B_{i}=B\left(P_{i}, \rho\right) \in \mathbb{R}^{2} \times \mathbb{R}^{+}$on which $\left|D_{0} \Phi\right|^{2}+\left|\dot{A}-\nabla A_{0}\right|^{2} \mid>\epsilon_{0}$ (by uniform continuity $\rho$ is independent of $P_{i}$ ). Therefore,

$$
\int_{\mathbb{R}^{2} \times \mathbb{R}^{+}}\left(\left|D_{0} \Phi\right|^{2}+\left|\dot{A}-\nabla A_{0}\right|^{2} \mid\right) d x d t \geq \sum_{i} \epsilon_{0}\left|B_{i}\right|=\infty
$$

contradicting the energy estimate (??). Thus $\left(A_{\infty}, \Phi_{\infty}\right)$ satisfies the static equations (1.2). 
Consider the winding number of the solution which as mentioned above is given by $\int_{\mathbb{R}^{2}} B d x$ and is an integer multiple of $2 \pi$. By the smoothness of $B$ and the exponential decay of theorem 4.1 we know that the winding number is constant for all time. In the case of $\lambda=1$ we also know the uniform exponential decay estimate of theorem 3.11. In fact, this implies that the limiting static solution $\left(A_{\infty}, \Phi_{\infty}\right)$ has the same winding number as the initial data. This is done by improving the weak convergence of the subsequence to a strong convergence. We will write $B_{\infty}=* d A_{\infty}$ for the magnetic field of the weak limit.

Theorem 5.2 (Strong Convergence I). Let $\lambda=1$ and let $(A, \Phi)$ be the solution in Coulomb gauge. Assume the initial data satisfy

$$
|B(x, 0)| \leq w(x, 0) \quad \text { and } \quad\left|D_{A(0)} \Phi(x, 0)\right| \leq 3 w(x, 0) .
$$

Then for $\left(t_{\nu}\right)$ the subsequence in the previous lemma,

$$
B\left(t_{\nu}\right) \rightarrow B_{\infty} \text { strongly in } L^{1} \cap L^{\infty}
$$

and thus the limiting static solution possesses the same winding number as the initial data,

$$
\begin{aligned}
& D_{A\left(t_{\nu}\right)} \Phi\left(t_{\nu}\right) \rightarrow D_{A_{\infty}} \Phi_{\infty} \text { strongly in } L^{1} \cap L^{\infty} \\
& w\left(t_{\nu}\right) \rightarrow w_{\infty} \text { strongly in } L^{1} \cap L^{\infty} \\
& \nabla B\left(t_{\nu}\right) \rightarrow \nabla B_{\infty} \text { strongly in } L^{1} \cap L^{\infty} \\
& D_{A\left(t_{\nu}\right)}^{2} \Phi\left(t_{\nu}\right) \rightarrow D_{A_{\infty}}^{2} \Phi_{\infty} \text { strongly in } L^{1} \cap L^{\infty} .
\end{aligned}
$$

In addition, for the same sequence $\left(t_{\nu}\right)_{\nu}$,

$$
\begin{gathered}
\left\|A\left(t_{\nu}\right)-A_{\infty}\right\|_{L^{r}\left(\mathbb{R}^{2}\right)} \longrightarrow 0 \text { for each } 2 \leq r \leq \infty \\
\lim _{t \rightarrow \infty}\left\|\left(A\left(t_{\nu}\right)-A_{\infty}\right)\right\|_{H^{2}\left(\mathbb{R}^{2}\right)}=0 .
\end{gathered}
$$

(Recall that in the Coulomb gauge $A(t), A_{\infty} \in L^{r}\left(\mathbb{R}^{2}\right)$ only for $2<r \leq \infty$ (c.f. theorems 3.10 and 5.1); however, the differences converge as above for $2 \leq r \leq \infty$.)

Proof. The strong convergence in $L^{1} \cap L^{\infty}$ follows from the uniform convergence on compact sets of these quantities together with their uniform exponential decay as $|x| \rightarrow \infty$ given by theorem 3.11. The convergence in 
(5.2) for $r>2$ follows from the convolution estimates for the integral (3.16) giving $A$ (see [14, p. 232]). For the case $r=2$ and (5.3) we use the fact that

$$
\int_{\mathbb{R}^{2}}\left(B\left(t_{\nu}\right)-B_{\infty}\right) d x=0
$$

which is true since $B\left(t_{\nu}\right) \rightarrow B_{\infty}$ in $L^{1}\left(\mathbb{R}^{2}\right)$ and $\int B\left(t_{\nu}\right) d x=2 \pi N$ where $N \in$ $\mathbf{Z}$ (see [19, Chaper Two]). The integral formula for $A\left(t_{\nu}\right)-A_{\infty}$ (see (3.16)) involves convolutions of the following type

$$
Q\left(x, t_{\nu}\right)=\int_{\mathbb{R}^{2}} \frac{x-y}{|x-y|^{2}} f_{\nu}(y) d y
$$

where $f_{\nu}=B\left(t_{\nu}\right)-B_{\infty}$. Using (5.4) we can write this as

$$
Q\left(x, t_{\nu}\right)=\int_{\mathbb{R}^{2}}\left(\frac{x-y}{|x-y|^{2}}-\frac{x}{|x|^{2}}\right) f_{\nu}(y) d y .
$$

Next we claim that

$$
|x|^{2}\left|Q\left(x, t_{\nu}\right)\right| \leq c_{1}|x|^{3} e^{-r \frac{|x|}{2}}\left\|e^{r|y|} f_{\nu}\right\|_{L^{\infty}(d y)}+c_{2}\left\|\left(1+|y|^{2}\right) f_{\nu}\right\|_{L^{1}(d y)} .
$$

To prove this we split up the integral into two parts $Q_{1}, Q_{2}$ on the inner region $I=\left\{|x-y|<\frac{|x|}{2}\right\}$ and outer region $O=\left\{|x-y|>\frac{|x|}{2}\right\}$ respectively:

$$
\begin{aligned}
Q_{1} & =\int_{I}\left(\frac{x-y}{|x-y|^{2}}-\frac{x}{|x|^{2}}\right) f_{\nu}(y) d y \\
Q_{2} & =\int_{O}\left(\frac{x-y}{|x-y|^{2}}-\frac{x}{|x|^{2}}\right) f_{\nu}(y) d y .
\end{aligned}
$$

To estimate the first term we use the fact that $y \in I$ implies $|y|>\frac{|x|}{2}$ so that $e^{-r \frac{|x|}{2}} e^{r|y|}>1$. To estimate the second term we use the fact that on $O$ $|x-y|>\frac{|x|}{2} \quad$ and so $\quad\left|\frac{|x|^{2}(x-y)-|x-y|^{2} x}{|x-y|^{2}}\right|=O\left(|y|^{2}\right)$ as $|x| \rightarrow \infty$.

Notice that if $r<1$ then $e^{r|x|}\left(B\left(t_{\nu}\right)-B_{\infty}\right)=e^{r|x|} f_{\nu}(x)$ goes to zero as $\nu \rightarrow \infty$ uniformly as a function of $x$ by the uniform in time exponential decay of $B($ as $\lambda=1)$. Therefore $\lim _{\nu \rightarrow \infty}|x|^{2} Q\left(x, t_{\nu}\right)=0$ uniformly in $x$ and so also $Q \rightarrow 0$ in $L^{2}$. This gives the convergence of $A\left(t_{\nu}\right) \rightarrow A_{\infty}$ in $L^{2}$. By once more differentiating the elliptic system for $A$ in (3.14) we obtain (5.3). 
We now ensure that (for $\lambda=1$ and in the Coulomb gauge) along the subsequence $\left(t_{\nu}\right)_{\nu}$ there exist gauge transformations $\chi\left(t_{\nu}\right), \chi_{\infty}$ such that $\rho\left(\chi\left(t_{\nu}\right)\left(A\left(t_{\nu}\right), \Phi\left(t_{\nu}\right)\right)-\rho\left(\chi_{\infty}\right)\left(A_{\infty}, \Phi_{\infty}\right)\right.$ becomes arbitrarily small in $H^{2}\left(\mathbb{R}^{2}\right)$ as $\nu \rightarrow \infty$.

Theorem 5.3 (Strong Convergence II). Let $\lambda=1,(A, \Phi)$ be the solution in Coulomb gauge and $t_{\nu}$ as before. There exist gauge transformations $\chi_{\nu}, \chi_{\infty} \in C^{\infty}\left(\mathbb{R}^{2}\right)$ such that if $\Psi^{\prime}\left(t_{\nu}\right)=\left(A^{\prime}\left(t_{\nu}\right), \Phi^{\prime}\left(t_{\nu}\right)\right)=$ $\rho\left(\chi_{\nu}\right)\left(A\left(t_{\nu}\right), \Phi\left(t_{\nu}\right)\right)$ and $\Psi_{*}=\rho\left(\chi_{\infty}\right)\left(A_{\infty}, \Phi_{\infty}\right)$ then

$$
\lim _{\nu \rightarrow+\infty}\left\|\Psi^{\prime}\left(t_{\nu}\right)-\Psi_{*}\right\|_{H^{2}\left(\mathbb{R}^{2}\right)}=0
$$

In addition, $\Psi^{\prime}\left(t_{\nu}\right), \Psi_{*}$ belong to $W^{k, \infty}\left(\mathbb{R}^{2}\right)$ for all positive integers $k$ and $d \chi_{\nu}, d \chi_{\infty}$ lie in $H^{k}\left(\mathbb{R}^{2}\right)$ for $k=0,1, \ldots$

Proof. On bounded sets $\Phi$ and its derivatives converge uniformly by the Arzela-Ascoli theorem. Therefore, the function $1-|\Phi|$ converges in $H^{2}$ since its first two derivatives converge uniformly on bounded sets and have exponential decay uniformly in time. Thus the issue is the behaviour of $\arg \Phi$ as $|x| \rightarrow \infty$. Restricting to the set $\left\{|\Phi|>\frac{1}{2}\right\}$ we may write $\Phi=|\Phi| e^{i f}$ where $f=\arg \Phi$ is well defined. Then

$$
D \Phi=(\nabla|\Phi|+i(\nabla f-A)|\Phi|) e^{i f}
$$

Using this and the similar formula for $D^{2} \Phi$ we see that $\Phi$ converges in $H^{2}$ if $f$ does. Since $\left|D^{\alpha} \Phi\right|$ for $|\alpha|=1,2$ and $w$ decay exponentially fast uniformly in time (cf. theorem 3.11), we have

$$
\sup _{t \geq 0}|\nabla f-A|+\left|\nabla^{2} f-\nabla A\right| \leq c(\epsilon) e^{-(1-\epsilon)|x|} .
$$

Thus as $\nu \rightarrow \infty$.

$$
(\nabla f-A)\left(t_{\nu}\right) \longrightarrow\left(\nabla f_{\infty}-A_{\infty}\right) \quad \text { in } H^{1}\left(\mathbb{R}^{2}\right)
$$

so that $\nabla f$ converges in $H^{1}$. Thus we are left to investigate the convergence of $f$ in $L^{2}$. It turns out that it is necessary to apply a gauge transformation to ensure that $f$ converges in $L^{2}$. To see this write out the convolution integral (3.16) for $A$ and collect terms in powers of $|x|^{-1}$ to obtain an asymptotic expansion for $A$ as $|x| \rightarrow \infty$. This is proved as in [19, Chap 6] or [38]. The components of $A$ are expressed using polar coordinates $(r, \theta)$ as

$$
A(x, t)=A^{(1)}(x, t)+A^{(2)}(x, t)+A^{(3)}(x, t)
$$




$$
\begin{aligned}
A_{i}(x, t) & =\frac{\sigma_{i j}}{2 \pi} \int_{\mathbb{R}^{2}} \frac{x_{j}-y_{j}}{|x-y|^{2}} B(y, t) d y \\
A_{i}^{(1)}(x, t) & =\sum_{j} \frac{1}{2 \pi} \frac{\partial \theta(x, t)}{\partial x_{i}} N \\
A_{i}^{(2)}(x, t) & =\sum_{j} \frac{\sigma_{i j}}{2 \pi} \int_{\mathbb{R}^{2}} \frac{2(x \cdot y) x_{j}-y_{j}|x|^{2}}{|x|^{4}} B(y, t) d y \\
& =\sum_{j, k} \sigma_{i j} \frac{2 x_{j} x_{k}-\delta_{j k}|x|^{2}}{|x|^{4}} \int_{\mathbb{R}^{2}} y_{k} B(y, t) d y \\
& =-\frac{1}{2 \pi} \sum_{k} \frac{\partial^{2} \theta(x, t)}{\partial x_{i} \partial x_{k}} \int_{\mathbb{R}^{2}} y_{k} B(y, t) d y
\end{aligned}
$$

and

$$
\left|A_{i}^{(3)}\left(x, t_{\nu}\right)\right| \leq \frac{C}{1+|x|^{3}} .
$$

Here $N$ is the winding number and $\sigma_{i j}$ the antisymmetric tensor with $\sigma_{12}=1$ for $i, j=1,2$. Along $t_{\nu}$,

$$
\left\|A^{(3)}\left(t_{\nu}\right)-A_{\infty}^{(3)}\right\|_{L^{2}\left(\mathbb{R}^{2}\right)} \longrightarrow 0 \quad \text { as } \nu \rightarrow \infty .
$$

Since $\nabla f-A$ decays exponentially independent of time we obtain by integration

$$
\begin{aligned}
& f\left(t_{\nu}\right)=N \theta+f^{(2)}\left(t_{\nu}\right)+f^{(3)}\left(t_{\nu}\right)+k\left(t_{\nu}\right) \\
& f(\infty)=N \theta+f^{(2)}(\infty)+f^{(3)}(\infty)+k(\infty)
\end{aligned}
$$

for constants $k\left(t_{\nu}\right)$ and $k(\infty)$ where $f^{(3)}\left(t_{\nu}\right) \leq \frac{c}{1+|x|^{2}}$ and converges to $f_{\infty}^{(3)}$ in $L^{2}\left(\mathbb{R}^{2}\right)$ and

$$
\begin{aligned}
& f^{(2)}\left(t_{\nu}\right)=-\frac{1}{2 \pi} \frac{\partial \theta}{\partial x_{k}} \int_{\mathbb{R}^{2}} y_{k} B\left(y, t_{\nu}\right) d y \\
& f^{(2)}(\infty)=-\frac{1}{2 \pi} \frac{\partial \theta}{\partial x_{k}} \int_{\mathbb{R}^{2}} y_{k} B(y, \infty) d y
\end{aligned}
$$

Since $f^{(2)}\left(t_{\nu}\right)+k\left(t_{\nu}\right)$ is not $L^{2}$-convergent we change to a different gauge to annihilate it: we define a gauge transformation by truncating $f^{(2)}$ to guarantee smoothness. At time $t_{\nu}$ we let

$$
\chi\left(x, t_{\nu}\right)=\frac{\eta(x)}{2 \pi} \frac{\partial \theta}{\partial x_{k}} \int_{\mathbb{R}^{2}} y_{k} B\left(t_{\nu}, y\right) d y \in C^{\infty}\left(\mathbb{R}^{2}\right)
$$


where $\eta$ is smooth, equal to zero in a neighbourhood $N_{1}$ of the origin and equal to one outside $N_{2}$, a larger neighbourhood of the origin (containing $\left.N_{1}\right)$. Define $\chi_{\infty}$ similarly. Then $f\left(t_{\nu}\right)+\chi\left(t_{\nu}\right)-k\left(t_{\nu}\right)-f_{\infty}-\chi_{\infty}+k(\infty)$ can be made arbitrarily small in $L^{2}$ by making $t_{\nu}$ large enough. After applying these gauge transformations $A^{\prime}\left(t_{\nu}\right)$ still converges in $H^{2}$. Finally the $W^{k, \infty}$ estimates follow from those of theorem 3.10.

\subsection{The Adiabatic Approximation.}

Let $\Psi(t)$ be the solution to the initial value problem for $(2.5)$ with $\lambda=1$. For any fixed $\epsilon>0$ theorem 5.3 provides a time $T_{0}$, a gauge transformation $g \in C^{\infty}\left(\mathbb{R}^{2}\right)$ and $\Psi_{*}$ (a critical point of $\mathcal{E}$ ), such that $\left\|\rho(g) \Psi\left(T_{0}\right)-\Psi_{*}\right\|_{H^{2}\left(\mathbb{R}^{2}\right)}<\epsilon$. At this time $T_{0}$ we change back to the temporal gauge and consider $\tilde{\Psi}(t)$ the solution of (2.5) with initial data $\rho(g) \Psi\left(T_{0}\right)$, i.e. $\tilde{\Psi}\left(t-T_{0}\right)=\rho(g) \Psi(t)$. For notational convenience we start measuring time at $T_{0}$, or equivalently we relabel $\tilde{\Psi}(t)$ as $\Psi(t)$ for the remainder of this section. We will revert to calling it $\tilde{\Psi}(t)$ in section 6 .

In this section we describe a method to prove that in the case of $\lambda=1$, $\Psi(t)$ converges to a static solution without passing to subsequences in time. The difficulty here is that there is an infinite dimensional manifold of equilibria and different subsequences might converge to different limits. The adiabatic approximation which we use to overcome this difficulty has a very clear geometrical interpretation which we now explain. Let $S_{N}$ be the of space smooth, finite action critical points (static solutions) of $\mathcal{E}$ which have winding number $N$. We will find at each time the point $\mathbb{P}(\Psi(t)) \in S_{N}$ which best approximates (in $L^{2}$ ) the solution $\Psi(t)$. The existence and uniqueness of $\mathbb{P}(\Psi(t))$ follows from theorem 5.15. One then obtains estimates which show that $\Psi(t)$ approaches $\mathbb{P}(\Psi(t))$ and that $\mathbb{P}(\Psi(t))$ has a limit as $t \rightarrow \infty$ which will be the limit of our solution. These estimates follow from the fact $S_{N}$ is energy minimizing. We prove the following theorem, which is the main result of this section:

Theorem 5.4 (Asymptotics in a Neighbourhoodof the Solution Space). Let $\lambda=1$. There exists a number $\epsilon_{*}=\epsilon_{*}(N)$ such that if $\epsilon<\epsilon_{*}$ the following is true. Let $\Psi \in C^{\infty}\left(\mathbb{R}^{2} \times(0, \infty) ; \mathbb{R}^{2} \times \mathbb{C}\right) \bigcap C\left(\mathbb{R}^{2} \times[0, \infty) ; \mathbb{R}^{2} \times \mathbb{C}\right)$ be the solution of (2.5) with smooth initial data $\Psi(0)=(A(0), \Phi(0))$ and satisfy the conditions

(i) $\mathcal{E}(A(0), \Phi(0))<+\infty$ and $(A(0), \Phi(0))$ has winding number $N$.

(ii) there exists $\Psi_{*} \in S_{N} \bigcap_{k \in \mathbb{N}} W^{k, \infty}\left(\mathbb{R}^{2} ; \mathbb{R}^{2} \times \mathbb{C}\right)$ such that $\left\|\Psi(0)-\Psi_{*}\right\|_{H^{2}}<$ 
$\epsilon$ and

(iii) $\Psi(t) \in \bigcap_{k \in \mathbb{N}} W^{k, \infty}\left(\mathbb{R}^{2} ; \mathbb{R}^{2} \times \mathbb{C}\right) \cap\left\{\Psi_{*}+H^{2}\left(\mathbb{R}^{2} ; \mathbb{R}^{2} \times \mathbb{C}\right)\right\}$ for all $t \geq 0$. Then the curve $\Psi(t)$ projects to a curve $\mathbb{P}(\Psi(t))$ in $S_{N}$ and the two curves converge in $H^{2}$ to a unique limit in $S_{N}$ as $t \rightarrow \infty$. Precisely, there exist in $S_{N} \bigcap_{k \in \mathbb{N}} W^{k, \infty}\left(\mathbb{R}^{2} ; \mathbb{R}^{2} \times \mathbb{C}\right) \cap\left\{\Psi_{*}+H^{2}\left(\mathbb{R}^{2} ; \mathbb{R}^{2} \times \mathbb{C}\right)\right\}$ a unique $\Psi_{\infty}$, and for all $t>0$ a unique $\mathbb{P}(\Psi(t))$ such that

$$
\begin{aligned}
& \|\Psi(t)-\mathbb{P}(\Psi(t))\|_{L^{2}}=\inf _{\Psi_{0} \in S_{N}}\left\|\Psi(t)-\Psi_{0}\right\|_{L^{2}} \\
& \|\Psi(t)-\mathbb{P}(\Psi(t))\|_{H^{2}} \leq C e^{-\delta t} \\
& \left\|\mathbb{P}(\Psi(t))-\Psi_{\infty}\right\|_{H^{2}} \leq C e^{-\delta t}
\end{aligned}
$$

for some positive numbers $C, \delta$.

The proof of this theorem appears in section 5.b.iv after some preliminary results. It is more convenient to work with a gauge transform $\Psi^{\#}(t)=$ $\rho(\chi(t)) \Psi(t)$ and then move back to the temporal gauge at the end. Thus we will show that there exists a time-dependent gauge transformation $\rho(\chi(t))$ such that $\Psi^{\#}(t)=\rho(\chi(t)) \Psi(t)$ converges in $H^{2}$ to a finite action solution of the static equations $\Psi_{\infty}^{\#}$. We will write $\Psi^{\#}(t)=\Psi_{0}(t)+\psi(t)$ where $\Psi_{0}(t) \in S_{N}$. The basic idea behind the proof is to show that the Hessian of $\mathcal{E}$ acts as a Liapunov functional and so $\psi(t) \rightarrow 0$ as $t \rightarrow \infty$ exponentially fast. There are difficulties with this, however, which we now discuss:

(i) Gauge Invariance. The gauge group $\mathcal{G} \equiv C^{\infty}\left(\mathbb{R}^{2} ; S^{1}\right)$ acts as an infinite dimensional symmetry group on $S_{N}$. The Hessian inherits from this an infinite dimensional null space (the tangent space to the orbit of $\mathcal{G}$ ). This is a difficulty which can be removed by use of appropriate gauge conditions which are described in sections 5.b.i and 5.b.iii. To this end it is convenient to introduce the Sobolev completions $S_{N}^{(2)}$ (static solutions) and $\mathcal{G}^{(3)}$ (gauge transformations) defined in (5.24) and (5.10). The gauge conditions amount to requiring that $\psi(t)$ be orthogonal to the tangent space to the orbit of $\Psi_{0}$ under $\mathcal{G}^{(3)}$, which is written as $T_{\Psi_{0}}\left(\mathcal{G}^{(3)} \cdot \Psi_{0}\right)$. This is possible by lemmas 5.7-5.9 which prove the existence of slices of the action of the gauge group.

(ii) The Moduli Space. Define the quotient $M_{N}=S_{N}^{(2)} / \mathcal{G}^{(3)}$ which is called the moduli space of gauge equivalence classes of the static solutions and let $\pi: S_{N}^{(2)} \rightarrow M_{N}$ be the natural projection. $S_{N}^{(2)}$ is a principal bundle over $M_{N}$ with structure group $\left.\mathcal{G}^{(3)}\right)$ and $M_{N}$ is a $2 N$-dimensional manifold (see [19] and section 5.b.ii). Even after factoring out the gauge symmetry we must deal with the degeneracy of the critical points of $\mathcal{E}$; this is reflected in the kernel of an elliptic operator $L_{\Psi_{0}}$ defined in (5.22) which defines the Hessian of $\mathcal{E}$ at $\Psi_{0}$. Dealing with this degeneracy amounts to choosing $\Psi_{0}(t)$ 
correctly as follows:

we seek a curve $\Psi_{0}(t) \in S_{N}^{(2)}$ so that $\psi(t)=\Psi^{\#}(t)-\Psi_{0}(t)$ is $L^{2}$-orthogonal to the tangent space to $S_{N}^{(2)}$ at $\Psi_{0}(t)$.

Lemma 5.12 tells us that this tangent space is given by

$$
T\left(\Psi_{0}\right) \equiv \operatorname{Ker} L_{\Psi_{0}} \oplus T_{\Psi_{0}}\left(\mathcal{G}^{(3)} \cdot \Psi_{0}\right)
$$

where $\operatorname{Ker} L_{\Psi_{0}}$ forms a horizontal subspace at $\Psi_{0}$ and (lemma 5.11) can be identified with the tangent space to $M_{N}$ at $\pi\left(\Psi_{0}\right)$. So in addition to the condition that $\psi$ be orthogonal to $T_{\Psi_{0}}\left(\mathcal{G}^{(3)} \cdot \Psi_{0}\right)$ mentioned in (i) above, this leads to the condition that $\psi$ be orthogonal to $\operatorname{Ker} L_{\Psi_{0}}$. The differential operator $L_{\Psi_{0}(t)}$ defines the Hessian of the functional (1.1) on the subspace orthogonal to the gauge flow. Thus these orthogonality conditions ensure the nondegeneracy of the Hessian, which can therefore be used as a Liapunov functional. In addition the orthogonality of $\psi$ with respect to $\operatorname{Ker} L_{\Psi_{0}}$ is equivalent to determining the curve $q(t)=\pi\left(\Psi_{0}(t)\right) \in M_{N}$ as a solution of a suitable ordinary differential equation (lemma 5.18). Then in lemma 5.13 $\Psi_{0}(t)$ is determined as the horizontal lift of $q(t)$. This means that $\frac{d \Psi_{0}}{d t}$ is $L^{2}$-orthogonal to $T_{\Psi_{0}}\left(\mathcal{G}^{(3)} \cdot \Psi_{0}\right)$.

Finally we draw the reader's attention to theorem 5.15. This is a tubular neighbourhood theorem which states that for a point $\Psi$ close enough to $S_{N}^{(2)}$ there is a unique point on $S_{N}^{(2)}$ which is $L^{2}$ closest to $\Psi$. We denote this point $\mathbb{P}(\Psi)$. The result is non-standard because the closest point is found in $L^{2}$ while the ambient space has norm $H^{2}$. The proof of the result depends on the fact that $S_{N}^{(2)}$ is defined by an elliptic system of equations (modulo gauge invariance). This leads to a regularity result (lemma 5.14) which allows us to effect the usual existence proof for tubular neighbourhoods (see e.g. [12, XVI.25]). Theorem 5.15 allows us to identify $\Psi_{0}(t)$ as the closest point on $S_{N}$ to $\Psi^{\#}(t)$. We then apply the gauge transformation $-\chi(t)$ to go back to temporal gauge and obtain the result stated in theorem 5.4 using the fact that $\mathbb{P}$ commutes with the action of the gauge group.

The remainder of this section is divided up as follows. In section 5.b.i we describe slices and gauge conditions. Next in section 5.b.ii we describe the manifold structure of the moduli space. We then give an ansatz for the solution and prove some preparatory lemmas in section 5.b.iii before giving the proof of theorem 5.4 in section 5.b.iv. Throughout section 5.b let $\Psi_{*}$ denote the point in $S_{N}$ described in theorem 5.4. 
5.2.i. Gauge Orthogonality and Slices. Recall that we have defined $S_{N} \equiv\left\{\Psi_{0}(A, \Phi) \in C^{\infty}\left(\mathbb{R}^{2} ; \mathbb{R}^{2} \times \mathbb{C}\right):\right.$

$\Psi_{0}$ is a finite action critical point of $\mathcal{E}$ with $\lambda=1$ of winding number $N\}$.

The gauge group $\mathcal{G} \equiv C^{\infty}\left(\mathbb{R}^{2} ; S^{1}\right)$ acts on $S_{N}$ and the orbit through $\Psi_{0}$ is defined by

$$
\mathcal{G} \cdot \Psi_{0} \equiv\left\{\rho(\chi) \Psi_{0}: e^{i \chi} \in C^{\infty}\left(\mathbb{R}^{2} ; S^{1}\right)\right\} .
$$

The space of orbits is called the moduli space, denoted by $M_{N}=S_{N} / \mathcal{G}$ which is identified with $S_{N}^{(2)} / \mathcal{G}^{(3)}$ by lemma 5.10. The space $M_{N}$ possesses a manifold structure of dimension $2 N$ (c.f. [19]). We let $\pi: S_{N} \rightarrow M_{N}$ be the natural projection. To obtain good estimates we must factor out the gauge symmetry. The standard way to do this is to find a slice of the action of $\mathcal{G}$ (see [11, 15]). This is most conveniently done in a Sobolev space setting, which explains the prevalence of Sobolev norms throughout this section. In the $C^{\infty}$ setting we define a slice through $\Psi_{0}=(a, \phi)$ to be the affine space $\Psi_{0}+\mathcal{S} \mathcal{L}_{\Psi_{0}}$ where

$$
\mathcal{S} \mathcal{L}_{a, \phi} \equiv \mathcal{S} \mathcal{L}_{\Psi_{0}} \equiv\left\{(\beta, \eta) \in C^{\infty}\left(\mathbb{R}^{2} ; \mathbb{R}^{2} \times \mathbb{C}\right): \nabla \cdot \beta-(i \phi, \eta)=0\right\} .
$$

Based on this we may define gauge orthogonality as follows:

Definition 1. We say that $(\beta, \eta) \in C^{\infty}\left(\mathbb{R}^{2} ; \mathbb{R}^{2} \times \mathbb{C}\right)$ is gauge orthogonal with respect to $(a, \phi) \in C^{\infty}\left(\mathbb{R}^{2} ; \mathbb{R}^{2} \times \mathbb{C}\right)$ if $(\beta, \eta) \in \mathcal{S} \mathcal{L}_{a, \phi}$.

Remarks. (i) Formally this means that $(\beta, \eta)$ is $L^{2}$-orthogonal to the tangent space to the orbit of the action of the gauge group $\mathcal{G}$ on $S_{N}$ at the point $(a, \phi)$, since for any compactly supported smooth function $\chi(x)$ we would then have

$$
\int_{\mathbb{R}^{2}} \beta \cdot \nabla \chi+(\eta, i \chi \phi)=0 .
$$

(ii) Notice that if $\Psi, \Psi^{b} \in C^{\infty}\left(\mathbb{R}^{2} ; \mathbb{R}^{2} \times \mathbb{C}\right)$ then $\left(\Psi-\Psi^{b}\right)$ is gauge orthogonal with respect to $\Psi$ if and only if it is gauge orthogonal with respect to $\Psi^{b}$.

We introduce a Sobolev space structure: there is a slight complication in that the boundary condition $\lim _{|x| \rightarrow \infty}|\Phi(x)|=1$ means that the static solutions are not contained in the usual Sobolev spaces. So instead let $\Psi_{*}=$ $\left(a_{*}, \phi_{*}\right) \in S_{N} \bigcap_{k \in \mathbb{N}} W^{k, \infty}\left(\mathbb{R}^{2} ; \mathbb{R}^{2} \times \mathbb{C}\right)$ be as in theorem 5.4 and introduce

$$
\mathcal{A}^{(2)} \equiv\left\{\Psi_{*}+\psi: \psi \in H^{2}\left(\mathbb{R}^{2} ; \mathbb{R}^{2} \times \mathbb{C}\right)\right\} .
$$


This is an affine space with tangent space at all points $\Psi_{0} \in \mathcal{A}^{(2)}$ given by

$$
T_{\Psi_{0}} \mathcal{A}^{(2)} \equiv\left\{\psi \in H^{2}\left(\mathbb{R}^{2} ; \mathbb{R}^{2} \times \mathbb{C}\right)\right\}
$$

Next introduce the gauge group in this context as

$$
\mathcal{G}^{(3)} \equiv\left\{e^{i \chi}: \chi \in H^{3}\left(\mathbb{R}^{2}\right)\right\} .
$$

Notice that this may be identified with the space of maps $s: \mathbb{R}^{2} \rightarrow \mathbb{C}$ such that $s-1 \in H^{3}$ and $|1+s|=1$ everywhere (see the proof of lemma 5.16). The manifold structure of $\mathcal{G}^{(3)}$ is determined by the exponential map

$$
\begin{aligned}
\operatorname{Exp}: H^{3}\left(\mathbb{R}^{2}\right) & \rightarrow \mathcal{G}^{(3)} \\
\chi & \mapsto e^{i \chi}
\end{aligned}
$$

Lemma 5.5 (Smoothness of the action). The action of the gauge group

$$
\begin{aligned}
\mathcal{G}^{(3)} \times \mathcal{A}^{(2)} & \rightarrow \mathcal{A}^{(2)} \\
(\chi,(A, \Phi)) & \mapsto\left(A+d \chi, \Phi e^{i \chi}\right)
\end{aligned}
$$

is a smooth free group action whose orbits have closed graphs in the sense that if $\left(A_{n}, \Phi_{n}\right) \rightarrow(A, \Phi)$ in $H^{2}$ and $\left(A_{n}+d \chi_{n}, \Phi e^{i \chi_{n}}\right) \rightarrow(a, \phi)$ in $H^{2}$ where $\left(\chi_{n}\right)_{n} \subset H^{3}$ then there exists $\chi \in H^{3}$ such that $\chi_{n} \rightarrow \chi$ in $H^{3}$ and $(a, \phi)=\left(A+d \chi, \Phi e^{i \chi}\right)$.

Proof. Since $\lim _{|x| \rightarrow \infty}|\Phi|=1$, the relation $\rho(\chi)(A, \Phi)=(A, \Phi)$ implies $e^{i \chi} \equiv 1$. Let $(A, \Phi)=\left(a_{*}+\beta, \phi_{*}+\eta\right)$. The map $(\beta, \chi) \mapsto \beta+d \chi$ is smooth. Thus we are left to consider $(\eta, \chi) \mapsto\left(\phi_{*}+\eta\right) e^{i \chi}$. Since we assume that $\Psi_{*}$ is in $W^{2, \infty}$ the map $\chi \mapsto \phi_{*}\left(e^{i \chi}-1\right)$ is smooth into $H^{2}$. The map $(\eta, \chi) \mapsto \eta\left(e^{i \chi}-1\right)$ is smooth by the Sobolev multiplication theorem. (The required estimates are given for example in [26]). To prove that the graphs of the orbits are closed notice first that $d \chi_{n} \rightarrow a-A$ in $H^{2}$ and so $\left\|d \chi_{n}\right\|_{L^{\infty}}$ are bounded. But also the fact that $|\phi|,|\Phi|$ have limit one as $|x| \rightarrow \infty$ implies, together with Rellich's theorem, that $e^{i \chi_{n}}-1$ converge in $H^{2}$. This implies that the $\chi_{n}$ tend to zero as $|x| \rightarrow \infty$ uniformly in $n$ from which one deduces immediately that $\chi_{n} \rightarrow \chi$ in $H^{3}$ where $(a, \phi)=\left(A+d \chi, \Phi e^{i \chi}\right)$.

The space $\mathcal{A}^{(2)}$ introduced above is in fact the total space of a principal fibre bundle under the gauge group action by $\mathcal{G}^{(3)}$ and base space

$$
\mathcal{M}^{(2)} \equiv \mathcal{A}^{(2)} / \mathcal{G}^{(3)}
$$


Endowed with the quotient topology which arises from the natural surjection, (which by a slight abuse of notation we write $\pi: \mathcal{A}^{(2)} \rightarrow \mathcal{M}^{(2)}$ ), it is Hausdorff (see the argument in [15] or [11]). In fact $\mathcal{M}^{(2)}$ inherits the structure of a smooth Hilbert manifold which we show by the next lemmas. (We also remark that using the Sobolev structure as above we obtain ultimately the same moduli space $M_{N}$. This is explained in section 5.b.ii.) We first define two spaces:

The tangent space to the orbit at $\Psi_{0}=(a, \phi)$ is the closed subspace of $T_{\Psi_{0}} \mathcal{A}^{(2)}$ given by

$$
T_{\Psi_{0}}\left(\mathcal{G}^{(3)} \cdot \Psi_{0}\right) \equiv\left\{(d \chi, i \phi \chi) \in T_{\Psi_{0}} \mathcal{A}^{(2)}: \chi \in H^{3}\left(\mathbb{R}^{2}\right)\right\}
$$

and the slice through $\Psi_{0}$ is the affine space $\Psi_{0}+\mathcal{S L}_{\Psi_{0}}^{(2)}$, where

$$
\mathcal{S L}_{\Psi_{0}}^{(2)} \equiv\left\{\psi=(\beta, \eta) \in H^{2}\left(\mathbb{R}^{2} ; \mathbb{R}^{2} \times \mathbb{C}\right): \nabla \cdot \beta-(i \phi, \eta)=0\right\}
$$

which is also a closed subspace of $T_{\Psi_{0}} \mathcal{A}^{(2)}$. The terminology slice is in the sense of the usual definition of a submanifold of the total space on which is given a smooth group action (see [11,15], Chapter 3). That is, there exists a neighbourhood $\mathcal{O}$ of $\Psi_{0}$ in $\mathcal{A}^{(2)}$ such that $\left(\Psi_{0}+\mathcal{S} \mathcal{L}_{\Psi_{0}}^{(2)}\right) \cap \mathcal{O}$ is a submanifold everywhere transverse to the action $\mathcal{G}^{(3)}$ on which the restriction of $\pi$ is a continuous open injection. The transversality to the action is in the sense that (locally) the tangent space to $\mathcal{A}^{(2)}$ (at each $\Psi$ ) has a direct sum decomposition into the tangent space of the orbit (at $\Psi$ ) and that of the slice through $\Psi_{0}$. The content of lemma 5.6 is precisely this decomposition, stated however only for $\Psi_{0}$ (which is all needed later on). For a general point $\Psi$ close to $\Psi_{0}$ a similar statement holds, namely,

$$
T_{\Psi} \mathcal{A}^{(2)}=T_{\Psi}\left(\mathcal{G}^{(3)} \cdot \Psi\right) \oplus \mathcal{S} \mathcal{L}_{\Psi_{0}}^{(2)} .
$$

The subsequent lemma 5.7 shows that the projection $\pi$ is locally a continuous injection. Finally as a corollary follows the manifold structure of $\mathcal{M}^{(2)}$.

Lemma 5.6 (Existence of Slice I). There exist $L^{2}$-orthogonal decompositions at $\Psi_{0}$,

$T_{\Psi_{0}} \mathcal{A}^{(2)}=T_{P s i_{0}}\left(\mathcal{G}^{(3)} \cdot \Psi_{0}\right) \oplus \mathcal{S} \mathcal{L}_{\Psi_{0}}^{(2)}\left(\right.$ and $\left.T_{P s i_{0}}\left(\mathcal{G}^{(3)} \cdot \Psi_{0}\right) \bigcap \mathcal{S}_{\Psi_{\Psi_{0}}^{(2)}}^{(2)}\{0\}\right)$.

The $L^{2}$-projection operator $\Theta_{\Psi_{0}}: T_{\Psi_{0}} \mathcal{A}^{(2)} \rightarrow \mathcal{S L}_{\Psi_{0}}^{(2)}$ varies continuously in the operator norm with respect to $\Psi_{0} \in \mathcal{A}^{(2)}$, i.e., there exists $c$ such that 
for $\left\|\Psi_{0}^{b}-\Psi_{0}^{\natural}\right\|_{H^{2}}$ small enough

$$
\left\|\left(\Theta_{\Psi_{0}^{b}}-\Theta_{\Psi_{0}^{\natural}}\right)(\beta, \eta)\right\|_{H^{2}} \leq c\left\|\Psi_{0}^{b}-\Psi_{0}^{\natural}\right\|_{H^{2}}\|(\beta, \eta)\|_{H^{2}}
$$

for all $(\beta, \eta) \in H^{2}\left(\mathbb{R}^{2} ; \mathbb{R}^{2} \times \mathbb{C}\right)$. Finally $\Theta_{\Psi_{0}^{b}}(\beta, \eta) \in H^{2}\left(\mathbb{R}^{2} ; \mathbb{R}^{2} \times \mathbb{C}\right)$ varies smoothly as a function of $(\beta, \eta) \in H^{2}\left(\mathbb{R}^{2} ; \mathbb{R}^{2} \times \mathbb{C}\right)$ and $\Psi_{0}^{b}$ in an $H^{2}$. neighbourhood of $\Psi_{0}$.

Proof. Let $\Psi_{0}=(a, \phi), \Psi^{b}=\left(a^{b}, \phi^{b}\right)$. Given $(\beta, \eta) \in T_{\Psi^{b}} \mathcal{A}^{(2)}$ we decompose $\beta=\beta^{\prime}+d \chi$ and $\eta=\eta^{\prime}+i \phi^{b} \chi$ and determine $\chi$ such that $\left(\beta^{\prime}, \eta^{\prime}\right) \in \mathcal{S L}_{\Psi^{b}}^{(2)}$. This leads to the equation

$$
-\Delta \chi+\left|\phi^{b}\right|^{2} \chi=\left(i \phi^{b}, \eta\right)+d^{*} \beta
$$

Consider this equation as a function $F\left(\chi, \Psi^{b},(\beta, \eta)\right)=0$ where $F: H^{3} \times$ $H^{2} \times H^{2} \rightarrow H^{1}$ is smooth and implicitly determines $\chi=g\left(\Psi^{b},(\beta, \eta)\right)$. The Fréchet derivative $D_{\chi} F\left(0, \Psi_{0}, 0\right)=\left(-\Delta+|\phi|^{2}\right): H^{3} \rightarrow H^{1}$ is a bounded linear isomorphism since $\lim _{|x| \rightarrow \infty}|\phi(x)|=1$. Therefore, by the implicit function theorem ([24]) we can write the solution of this equation as

$$
\chi=g\left(\Psi^{b},(\beta, \eta)\right)
$$

where $g: U \times H^{2}\left(\mathbb{R}^{2} ; \mathbb{R}^{2} \times \mathbb{C}\right) \rightarrow H^{3}\left(\mathbb{R}^{2}\right)$ is a smooth function for $\Psi^{b}$ in some open set $U=\left\{\left\|\Psi^{b}-\Psi_{0}\right\|_{H^{2}}<\delta\right\} \subset \mathcal{A}^{(2)}$ and linear in $(\beta, \eta)$. Also $g$ is linear with respect to $(\beta, \eta)$. This implies by the uniform boundedness principle $\left\|D_{1} g\left(\Psi^{\mathrm{b}},(\beta, \eta)\right)\right\|_{\mathcal{L}\left(H^{2}, H^{3}\right)} \leq c\|(\beta, \eta)\|_{H^{2}}$ for $\Psi^{\mathrm{b}} \in O$. From this we deduce (by the fundamental theorem of calculus)

$$
\left\|g\left(\Psi^{b},(\beta, \eta)\right)-g\left(\Psi^{\natural},(\beta, \eta)\right)\right\|_{H^{3}} \leq c\left\|\Psi^{b}-\Psi^{\natural}\right\|_{H^{2}}\|(\beta, \eta)\|_{H^{2}}
$$

which implies the continuity of $\Theta_{\Psi_{0}}$ as required.

Lemma 5.7 (Existence of Slice II). Let $\Psi_{0}=(a, \phi) \in \mathcal{A}^{(2)}, \Psi=$ $(A, \Phi) \in \mathcal{A}^{(2)}$ be such that $\left\|\Psi-\Psi_{0}\right\|_{H^{2}}<\epsilon$. For $\epsilon>0$ small enough $\Psi$ can be mapped by a gauge transformation uniquely onto the slice through $\Psi_{0}$. That is, there exist positive numbers $M, \epsilon_{1}$ depending only on $\Psi_{0}$ such that if $\epsilon<\epsilon_{1}$ there exists a unique gauge transformation $\chi \in H^{3}\left(\mathbb{R}^{2}\right)$ such that if $\Psi^{\#}=\left(A^{\#}, \Phi^{\#}\right)=\rho(\chi)(A, \Phi)$ then

$$
\Psi^{\#}-\Psi_{0} \in \mathcal{S} \mathcal{L}_{\Psi_{0}}^{(2)}
$$


and $\left\|\Psi^{\#}-\Psi_{0}\right\|_{H^{2}} \leq M \epsilon$. If also $\Psi_{0} \in C^{\infty}\left(\mathbb{R}^{2} ; \mathbb{R}^{2} \times \mathbb{C}\right)$ and $\Psi \in$ $\bigcap_{k \in \mathbb{N}} W^{k, \infty}\left(\mathbb{R}^{2} ; \mathbb{R}^{2} \times \mathbb{C}\right)$ then also $\Psi^{\#} \in \bigcap_{k \in \mathbb{N}} W^{k, \infty}\left(\mathbb{R}^{2} ; \mathbb{R}^{2} \times \mathbb{C}\right)$. The mapping

$$
\left(\Psi-\Psi_{0}\right) \mapsto\left(\Psi^{\#}-\Psi_{0}\right)
$$

between neighbourhoods of the origin in $H^{2}$ and $\mathcal{S L}_{\Psi_{0}}^{(2)}$ is smooth. Furthermore, the orbits through points close to $\Psi_{0}$ locally intersect the slice through $\Psi_{0}$ at most once. That is, there exists $\delta>0$ such that if $\Psi^{b} \neq \Psi^{\natural}$ (both in $\left.\mathcal{A}^{(2)}\right)$ satisfy

(i) $\left\|\Psi^{b}-\Psi_{0}\right\|_{H^{2}}+\left\|\Psi^{\natural}-\Psi_{0}\right\|_{H^{2}}<\delta$

(ii) $\left(\Psi^{b}-\Psi_{0}\right) \in \mathcal{S} \mathcal{L}_{\Psi_{0}}^{(2)}$ and $\left(\Psi^{\natural}-\Psi_{0}\right) \in \mathcal{S L}_{\Psi_{0}}^{(2)}$

then $\Psi^{\natural} \notin \mathcal{G}^{(3)} \cdot \Psi^{b}$.

Proof. Let $\Psi_{0}=(a, \phi)$ and $\Psi=\Psi_{0}+(\beta, \eta)$. We wish to find a real valued function $\chi$ such that

$$
\left(\beta+d \chi,(\phi+\eta) e^{i \chi}-\phi\right)=\left(\beta+d \chi, i \phi \chi+\eta e^{i \chi}+\phi\left(e^{i \chi}-1-i \chi\right)\right)
$$

is gauge orthogonal with respect to $(a, \phi)$. This leads to the following equation

$$
-\Delta \chi+|\phi|^{2} \chi=-\left(i \phi, \eta e^{i \chi}\right)-\left(i \phi, \phi\left(e^{i \chi}-1-i \chi\right)\right)+\nabla \cdot \beta .
$$

Consider this equation as the function $F(\chi, \beta, \eta)=0$ where $F: H^{3} \times H^{2} \times$ $H^{2} \rightarrow H^{1}$ is smooth and implicitly determines $\chi=g(\beta, \eta)$. The Fréchet derivative $D_{\chi} F(0,0,0)=\left(-\Delta+|\phi|^{2}\right): H^{3} \rightarrow H^{1}$ is a bounded linear isomorphism since $\lim _{|x| \rightarrow \infty}|\phi|=1$. Therefore, by the implicit function theorem ([24]) there is a smooth function $g$ between neighbourhood $U_{1}$ of the origin in $H^{2}\left(\mathbb{R}^{2} ; \mathbb{R}^{2} \times \mathbb{C}\right)$ and $U_{2}$ in $H^{3}\left(\mathbb{R}^{2}\right)$

$$
\begin{aligned}
& g: U_{1} \longrightarrow U_{2} \\
& (\beta, \eta) \quad \mapsto g(\beta, \eta)
\end{aligned}
$$

such that $F(g(\beta, \eta), \beta, \eta)=0$ and $g(0,0)=0$. Since $g$ is smooth it has a bounded derivative $g^{\prime}(0,0): H^{2} \rightarrow H^{3}$, let $\left\|g^{\prime}(0,0)\right\| \leq K$. Then $\exists \epsilon_{1}>0$ such that

$$
\|(\beta, \eta)\|_{H^{2}}<\epsilon_{1} \text { implies }\|g(\beta, \eta)\|_{H^{3}} \leq(1+K)\|(\beta, \eta)\|_{H^{1}} .
$$


By the smoothness of the gauge group action (lemma 5.5) this implies that

$$
\begin{aligned}
\left\|\Psi^{\natural}-\Psi_{0}\right\|_{H^{2}} & \leq\left\|\Psi^{\natural}-\Psi\right\|_{H^{2}}+\left\|\Psi-\Psi_{0}\right\|_{H^{2}} \\
& \leq c(1+K) \epsilon+\epsilon
\end{aligned}
$$

which gives (5.12). The $W^{k, \infty}$ statement follows from lemma B.2 applied to (5.13).

To prove the final part of the theorem, assume that on the contrary $\Psi^{b}=\left(A^{b}, \Phi^{b}\right)$ and $\Psi^{\natural}=\rho(\chi) \Psi^{b}=\left(A^{b}+d \chi, \Phi^{b} e^{i \chi}\right)$ are both gauge orthogonal with respect to $\Psi_{0}$. We will obtain a contradiction by showing that $\chi \equiv 0$. It follows from the gauge orthogonality conditions that

$$
\nabla \cdot \nabla \chi-\left(i \phi, \Phi^{b}\left(e^{i \chi}-1\right)\right)=0
$$

or

$$
-\Delta \chi+|\phi|^{2} \sin \chi=-\left(i \phi,\left(\Phi^{b}-\phi\right)\left(e^{i \chi}-1\right)\right)
$$

Since both $\Phi^{b}-\phi$ and $e^{i \chi} \Phi^{b}-\phi$ are in $H^{2}$, they approach zero uniformly as $|x| \rightarrow \infty$ (see [19, lemma 3.7.5 and the Rellich embedding]). But $\left|\Phi^{b}\right|,|\phi|$ have limit one as $|x| \rightarrow \infty$ and therefore $\chi$ converges uniformly to $0 \bmod 2 \pi$ as $|x| \rightarrow \infty$. Without loss of generality we may assume this to be zero: for if the limit is $2 \kappa \pi$ then consider $\tilde{\chi}=\chi-2 \kappa \pi$ which has limit zero asymptotically and note that $\Psi^{\natural}=\rho(\tilde{\chi}) \Psi^{b}$ and (5.16) holds with $\tilde{\chi}$ in place of $\chi$. Hence it suffices to show that $\tilde{\chi} \equiv 0$ and this justifies our assumption on $\chi$. Then

Fix $R>0$ such that on $\{|x|>R\}$ we have $\left|\Phi^{b}\right|>\frac{1}{2}$ and $\left|\frac{\sin \chi(x)}{\chi}\right|>\frac{1}{2}$.

$$
\left|\Phi^{b}\left(e^{i \chi}-1\right)\right| \geq\left|\Phi^{b}\right||\sin \chi| \geq \frac{1}{4}|\chi| \text { on }\{|x|>R\}
$$

By the assumption that $\Psi^{b}$ and $\Psi^{\natural}$ are gauge equivalent,

$$
\left\|\Psi^{\natural}-\Psi^{b}\right\|_{H^{2}}=\|d \chi\|_{H^{2}}+\left\|\Phi^{b}\left(e^{i \chi}-1\right)\right\|_{H^{2}}<2 \delta
$$

where $\delta>0$ is chosen small and is to be specified below. By the embedding $H^{2} \hookrightarrow L^{\infty},|d \chi|<2 \delta$ on $\mathbb{R}^{2}$. But also (5.17)- (5.18) imply $|\chi|<8 \delta$ for $|x|>R$ and altogether we have $|\chi| \leq k \delta$ for some constant $k$ on all of $\mathbb{R}^{2}$. We now choose $\delta$ small enough so that the last estimate for $\chi$ implies $v(x)=\frac{|\phi|^{2} \sin \chi}{\chi} \geq 0$ for all $x \in \mathbb{R}^{2}$. 
Returning to the equation (5.16) rewritten as $-\Delta \chi+v(x) \chi=f$ we note that $v(x) \rightarrow 1$ as $|x| \rightarrow \infty$ and the elliptic estimate below then holds,

$$
\|\chi\|_{H^{2}} \leq c\|f\|_{L^{2}} \leq c^{\prime}\|\chi\|_{L^{\infty}}^{2}\left\|\Phi^{b}-\phi\right\|_{L^{2}}^{2} \leq c^{\prime \prime} \delta^{2}\|\chi\|_{H^{2}} .
$$

So if $\delta$ is further chosen so that $c^{\prime \prime} \delta^{2}<1$ the above estimate yields $\chi \equiv 0$ on $\mathbb{R}^{2}$ as claimed, so $\Psi^{b}=\Psi^{\natural}$.

Corollary 5.8 (Manifold structure on $\mathcal{M}^{(2)}$ ). Fix $\Psi \in \mathcal{A}^{(2)}$, then there exists a neighbourhood $U_{1}$ of $\Psi$ in $\mathcal{A}^{(2)}$ and a neighbourhood $U_{2} \times U_{3}$ of $(0, i d)$ in $\mathcal{S} \mathcal{L} l_{\Psi}^{(2)} \times \mathcal{G}^{(3)}$ and a diffeomorphism

$$
\Xi: U_{1} \rightarrow U_{2} \times U_{3} .
$$

Furthermore the map $\tilde{G}: U_{2} \rightarrow \tilde{G}\left(U_{2}\right) \subset \mathcal{M}^{(2)}$ given by the restriction of $\pi \circ \Xi^{-1}$ to $U_{2} \times\{i d\}$ is an injection onto an open neighbourhood of $\pi(\Psi)$ in $\mathcal{M}^{(2)}$. Thus $\mathcal{M}^{(2)}$ inherits a smooth manifold structure under which it is locally diffeomorphic to the slices $\mathcal{S L}_{\Psi}^{(2)}$. Indeed, if we choose small open sets in $\mathcal{M}^{(2)}$ around $\pi(\Psi)$ then $\tilde{G}^{-1}$ gives a local chart. The transition functions between charts will be smooth by lemma 5.7 .

Proof. This is essentially a restatement of lemma 5.7. We define

$$
\Xi(\Psi+\psi)=\left(\Xi_{1}(\Psi+\psi), \Xi_{2}(\Psi+\psi)\right)=(\rho(g(\psi))(\Psi+\psi)-\Psi, g(\psi))
$$

where $g$ is as in the proof of lemma 5.7. The map $\Xi$ is smooth on an appropriate neighbourhood and has smooth inverse given by

$$
\Xi^{-1}(v, g)=\rho(g)^{-1}(v+\Psi)
$$

The fact that the map $\tilde{G}$ is one-to-one follows from the last statement of the lemma 5.7. The injectivity of the map $\tilde{G}^{-1}$ implies that locally two points on the slice through $\Psi$ correspond to different orbits, i.e. map onto two different points of $\mathcal{M}^{(2)}$. From the definition of the quotient topology $\tilde{G}$ is open and so a homeomorphism and thus gives a chart.

We now show that the slices of lemma 5.7 vary smoothly with $\Psi_{0}$.

Lemma 5.9 (Smooth variation of slices). Let $\Psi_{0}=(a, \phi) \in$ $S_{N}^{(2)} \bigcap_{k \in \mathbb{N}} W^{k, \infty}\left(\mathbb{R}^{2} ; \mathbb{R}^{2} \times \mathbb{C}\right)$ and let $\epsilon_{1}$ be as in lemma 5.7 . There exist positive numbers $\epsilon_{2}<\epsilon_{1}, \delta_{2}, L_{1}, L_{2}$ (depending only on $\Psi_{0}$ ) such that if $\delta<\delta_{2}$ 
the following is true. There exist neighbourhoods of the origin $U_{1}, U_{2}$ in $H^{2}\left(\mathbb{R}^{2} ; \mathbb{R}^{2} \times \mathbb{C}\right)$ and $U_{3}$ in $H^{3}\left(\mathbb{R}^{2}\right)$ and a smooth map

$$
\begin{aligned}
s: U_{1} \times U_{2} & \rightarrow U_{3} \\
\left(\left(F-\Psi_{0}\right), v\right) & \mapsto s\left(F-\Psi_{0}, v\right)
\end{aligned}
$$

such that

(i) if $\chi=s\left(F-\Psi_{0}, v\right)$ then $\rho(\chi) F-\left(\Psi_{0}+v\right) \in \mathcal{S L}_{\left(\Psi_{0}+v\right)}^{(2)}$.

(ii) $U_{1}$ contains the ball $\left\{\left\|F-\Psi_{0}\right\|_{H^{2}}<\epsilon_{2}\right\}$ and $U_{2}$ contains $\left\{\|v\|_{H^{2}}<\delta_{2}\right\}$.

(iii) if $\left(F-\Psi_{0}\right) \in \mathcal{S L}_{\Psi_{0}}^{(2)}$ then

$$
\|v\|_{H^{2}}<\delta \text { implies }\|\chi\|_{H^{3}}<L_{1} \delta \text { and }\|\rho(\chi) F-F\|_{H^{2}}<L_{2} \delta
$$

(where $L_{1}, L_{2}$ are independent of $\delta$ ).

Proof. We proceed exactly as in the proof of lemma 5.7, so let $\left(F-\Psi_{0}\right)=$ $(\beta, \eta)$ and $v=\left(v_{1}, v_{2}\right)$. Since $v_{2} \in H^{2}$ it follows that $\lim _{|x| \rightarrow \infty}|v(x)|=0$. The condition in (i) above leads to the following equation for $\chi=s(F-$ $\left.\Psi_{0}, v\right)$ :

$$
\left(-\Delta+|\phi|^{2}\right) \chi+v_{2} \phi \chi+\left(i\left(\phi+v_{2}\right), \eta e^{i \chi}-v_{2}+\phi\left(e^{i \chi}-1-i \chi\right)\right)-\nabla \cdot\left(\beta-v_{1}\right)=0 .
$$

Consider this as an equation $G\left(\chi, F-\Psi_{0}, v\right)=0$ where $G$ is a smooth function $H^{3} \times H^{2} \times H^{2} \rightarrow H^{1}$. (Recall that $\Psi_{0}$ is fixed.) The derivative of this map with respect to $\chi$ at the origin is the bounded isomorphism $\left(-\Delta+|\phi|^{2}\right): H^{3} \rightarrow H^{1}$, so by the implicit function theorem there is a $\chi=s\left(F-\Psi_{0}, v\right)$ satisfying property $(i)$. To complete the proof notice that by lemma 5.7 if $F-\Psi_{0} \in \mathcal{S} \mathcal{L}_{\Psi_{0}}^{(2)}$ then $s\left(F-\Psi_{0}, 0\right)=0$. Since $s$ is smooth we get the final estimates by taking $\delta_{2}, \epsilon_{2}$ small enough.

5.2.ii. The Moduli Space. Next we are interested in obtaining a local diffeomorphism between the moduli space $M_{N}$ and the subset of $\mathcal{S} \mathcal{L}_{\Psi_{0}}^{(2)}$ which consists of the critical points of $\mathcal{E}$ (for $\lambda=1$ ). We refer to images of open sets of $M_{N}$ under this map as local families of solutions. In lemma 5.11 their existence is proved and they are shown to form a submanifold modelled on the linear space $\operatorname{Ker} L_{\Psi_{0}}$ defined in (5.23) (which is locally diffeomorphic to $\left.M_{N}\right)$.

The moduli space $M_{N}$ was defined as the space of gauge equivalence classes of smooth finite energy solutions of the static equations (i.e., the 
critical points of the functional $\mathcal{E}(A, \Phi)$ (with $\lambda=1$ ) having winding number $N)$. It is known ([19]) that all the critical points of $\mathcal{E}$ are minima and $M_{N}$ can be identified with $N$-tuples of points in the plane $\left(Z_{1}, \ldots, Z_{N}\right)$, which are the (only) zeros of $\Phi$. (Notice that the zeros are gauge invariant. If a subset of the $Z_{i}$ are coincident there is a zero of higher multiplicity. The winding number is the total number of zeros of $\Phi$ counted with multiplicity). The proof of existence for these solutions depends crucially on the fact ([9]) that when $\lambda=1$ we can, for $N>0$, decompose the functional as

$$
\mathcal{E}(A, \Phi)=\frac{1}{2} \int_{\mathbb{R}^{2}}\left(4\left|\bar{\partial}_{A} \Phi\right|^{2}+\left(B+\frac{1}{2}\left(|\Phi|^{2}-1\right)\right)^{2}\right) d x+\pi N
$$

where $\bar{\partial}_{A} \Phi=\frac{1}{2}\left(D_{1}+i D_{2}\right) \Phi$. Thus the minima will be solutions of the first order Bogomol'nyi, or self-dual, equations

$$
\begin{aligned}
& \bar{\partial}_{A} \Phi=0 \\
& B+\frac{1}{2}\left(|\Phi|^{2}-1\right)=0 .
\end{aligned}
$$

(There are sign changes if $N<0$.) The existence theory for these equations is discussed in the book [19]. We shall write these equations schematically as

$$
\mathcal{B}(A, \Phi)=0 .
$$

Since the solution is invariant under interchange of any two of these $Z_{i}$ the moduli space is a symmetric product of $N$ copies of the complex plane $S^{N}(\mathbb{C})$. It is endowed with a natural metric and complex structure which makes it a Kähler manifold ([3],[34]).

Let the operator $L_{\Psi_{0}}$ be defined in terms of the Hessian of $\mathcal{E}$ at

$$
\Psi_{0} \in S_{N}^{(2)} \bigcap_{k \in \mathbb{N}} W^{k, \infty}\left(\mathbb{R}^{2} ; \mathbb{R}^{2} \times \mathbb{C}\right)
$$

by

$$
\left(\psi, L_{\Psi_{0}} \psi\right)_{L^{2}}=\left.\frac{d^{2}}{d s^{2}}\right|_{s=0} \mathcal{E}\left(\Psi_{0}+s \psi\right)+\int_{\mathbb{R}^{2}}(\nabla \cdot \beta-(i \phi, \eta))^{2}
$$

for $\psi=(\beta, \eta) \in C_{0}^{\infty}\left(\mathbb{R}^{2} ; \mathbb{R}^{2} \times \mathbb{C}\right)$. Notice that the second term on the right hand side is the expression occuring in the definition of $\mathcal{S L}_{\Psi_{0}}^{(2)}$ in definition 1. Thus we may say that $L_{\Psi_{0}}$ is obtained by linearising the second order static 
equations on $\mathcal{S} \mathcal{L}_{\Psi_{0}}^{(2)} . L_{\Psi_{0}}$ has the following properties for $\Psi_{0} \in W^{1, \infty}\left(\mathbb{R}^{2} ; \mathbb{R}^{2} \times\right.$ $\mathbb{C})$ (see [37] and appendix B):

(i) it is elliptic and the principal symbol is the Laplacian

(ii) it is non-negative and in fact can be factorized into a product of first order operators

$$
L_{\Psi_{0}}=\mathcal{D}_{\Psi_{0}}^{*} \mathcal{D}_{\Psi_{0}}
$$

where $\mathcal{D}_{\Psi_{0}}^{*}$ is the formal $L^{2}$-adjoint of $\mathcal{D}_{\Psi_{0}}$. The operator $\mathcal{D}_{\Psi_{0}}$ is obtained by the linearisation of the first order static equations above. These both extend to Fredholm operators from $H^{1} \rightarrow L^{2}$ (i.e. they have closed range and finite dimensional kernel and cokernel). The precise form of these operators is given in appendix $\mathrm{B}$.

(iii) it has a $2 N$-dimensional null space

$$
\operatorname{Ker} L_{\Psi_{0}} \equiv\left\{\psi \in H^{2}\left(\mathbb{R}^{2} ; \mathbb{R}^{2} \times \mathbb{C}\right): L_{\Psi_{0}} \psi=0\right\}
$$

where $N$ is the winding number of $\Psi_{0}$. In fact $\mathcal{D}_{\Psi_{0}}$ has a $2 N$-dimensional null space and $\mathcal{D}_{\Psi_{0}}^{*}$ has a trivial null space. This space can be identified with the tangent space to $M_{N}$ at $\pi\left(\Psi_{0}\right)$ (see lemma 5.11). (iv) if $(\psi, n)_{L^{2}}=0 \forall n \in K \operatorname{Ker} L_{\Psi_{0}}$ then there exists a number $\gamma$ such that

$$
\left(\psi, L_{\Psi_{0}} \psi\right)_{L^{2}} \geq \gamma\|\psi\|_{H^{1}}^{2}
$$

Definition 2. For $\Psi_{0}$ as above let $\operatorname{Ker} L_{\Psi_{0}}^{\perp}$ be the $L^{2}$-orthogonal subspace of $\operatorname{Ker} L_{\Psi_{0}}$, i.e.,

$$
\operatorname{Ker} L_{\Psi_{0}}^{\perp} \equiv\left\{\psi \in L^{2}\left(\mathbb{R}^{2} ; \mathbb{R}^{2} \times \mathbb{C}\right):(\psi, n)_{L^{2}\left(\mathbb{R}^{2}\right)}=0 \forall n \in \operatorname{Ker} L_{\Psi_{0}}\right\} .
$$

We have defined the solution space as $S_{N}=\{\mathcal{B}(A, \Phi)=0\} \cap C^{\infty}\left(\mathbb{R}^{2} ; \mathbb{R}^{2} \times\right.$ $\mathbb{C})$ where $\mathcal{B}$ is the set of Bogomol'nyi equations given in (5.20-5.21). The moduli space was then defined by $M_{N}=S_{N} / \mathcal{G}$. One may instead consider

$$
S_{N}^{(2)}=\{\mathcal{B}(A, \Phi)=0\} \cap \mathcal{A}^{(2)}
$$

and the corresponding moduli space $M_{N}^{(2)}=S_{N}^{(2)} / \mathcal{G}^{(3)}$.

Lemma 5.10. There is a bijection between $M_{N}$ and $M_{N}^{(2)}$. For our purposes the manifold structure on $M_{N}$ is inherited from that of $M_{N}^{(2)}$ (determined by the map $F$ in lemma 5.11) and we will use only the notation $M_{N}$. 
Proof. See appendix B.

Our next result gives local families of static $H^{2}$ solutions as a $2 N$ dimensional local submanifold of $\mathcal{S} \mathcal{L}_{\Psi_{0}}^{(2)}$. Recall that the Bogomol'nyi equations, which we write schematically as $\mathcal{B}(A, \Phi)=0$, are gauge invariant and so descend to a set of equations on $\mathcal{M}^{(2)}$. As remarked above, the moduli space $M_{N}$ is precisely $\left(\mathcal{B}^{-1}(0) \cap \mathcal{A}^{(2)}\right) / \mathcal{G}^{(3)}$. Via corollary 5.8 we can locally identify $M_{N}$ with the solutions of the Bogomol'nyi equations on a slice. Thus we make a definition:

Definition 3. For $\Psi_{0}$ as above let

$$
\tilde{M}_{N}\left(\Psi_{0}\right)=\left\{\Psi \in \mathcal{A}^{(2)}: \mathcal{B}(\Psi)=0 \text { and } \Psi-\Psi_{0} \in \mathcal{S L}_{\Psi_{0}}^{(2)}\right\} .
$$

Remark. The tangent space to $\tilde{M}_{N}\left(\Psi_{0}\right)$ at the point $\Psi_{0}$ can be identified with $\operatorname{Ker} L_{\Psi_{0}}$. This is clear because the operator $\mathcal{D}_{\Psi_{0}}$ is obtained by linearisation of the equations $\mathcal{B}(\Psi)=0$ on $\mathcal{S L}_{\Psi_{0}}^{(2)}$ while the kernel of $L_{\Psi_{0}}$ coincides with that of $\mathcal{D}_{\Psi_{0}}$ (see appendix B).

The next lemma gives a local diffeomorphism betweem $M_{N}, \tilde{M}_{N}\left(\Psi_{0}\right)$ and an open set of the linear space $\operatorname{Ker} L_{\Psi_{0}}$.

Lemma 5.11 (Local Families of Solutions). Let $\Psi_{0}=(a, \phi) \in$ $S_{N}^{(2)} \bigcap_{k \in \mathbb{N}} W^{k, \infty}\left(\mathbb{R}^{2} ; \mathbb{R}^{2} \times \mathbb{C}\right)$ and let $\pi: S_{N}^{(2)} \rightarrow M_{N}$ be the natural projection. Then $M_{N}, \operatorname{Ker} L_{\Psi_{0}}$ and $\tilde{M}_{N}\left(\Psi_{0}\right)$ are locally diffeomorphic as follows: there exist a neighbourhood $U_{1} \subset M_{N}$ of $\pi\left(\Psi_{0}\right)$, a neighbourhood $U_{2} \subset K e r L_{\Psi_{0}}$ of the origin and a neighbourhood $U_{3} \subset \tilde{M}_{N}\left(\Psi_{0}\right)$ of $\Psi_{0}$, and diffeomorphisms

$$
F: U_{1} \rightarrow U_{2} \quad G: U_{1} \rightarrow U_{3} \quad \tilde{\Psi}_{0}: U_{2} \rightarrow U_{3}
$$

such that $F\left(\pi\left(\Psi_{0}\right)\right)=0, G\left(\pi\left(\Psi_{0}\right)\right)=\Psi_{0}, \tilde{\Psi}_{0}(0)=\Psi_{0},\left.(G \circ \pi)\right|_{U_{3}}=\left.i d\right|_{U_{3}}$ and $\left.(\pi \circ G)\right|_{U_{1}}=\left.i d\right|_{U_{1}}$. The map

$$
\tilde{\Psi}_{0}: q \in U_{2} \mapsto \tilde{\Psi}_{0}(q)
$$

will be referred to as a local family of solutions. It satisfies $\tilde{\Psi}_{0}(q) \in$ $\bigcap_{k \in \mathbb{N}} W^{k, \infty}\left(\mathbb{R}^{2} ; \mathbb{R}^{2} \times \mathbb{C}\right)$. Finally, restricting the differential of the projection map to $K e r L_{\Psi_{0}}$ gives an isomorphism

$$
\pi_{*}: \operatorname{Ker} L_{\Psi_{0}} \rightarrow T_{\pi\left(\Psi_{0}\right)} M_{N}
$$

which we shall use to identify these spaces. (The space $T_{\pi\left(\Psi_{0}\right)} M_{N}$ is the tangent space to $M_{N}$ at $\pi\left(\Psi_{0}\right)$.) 
Proof. First we will construct $\tilde{\Psi}_{0}$. Starting with the static solution $\Psi_{0}$, we look for solutions, elements of $\tilde{M}_{N}\left(\Psi_{0}\right)$, in the form $\Psi_{0}+\psi$ and solve for $\psi$ using (5.20-5.21) and the gauge orthogonality conditions. It follows that $\psi$ satisfies a system of equations of the form

$$
\mathcal{D}_{\Psi_{0}} \psi=g(\psi)
$$

where $g(\psi)$ is quadratic in components of $\psi$ (see appendix B for an explicit formula). From this we get

$$
L_{\Psi_{0}} \psi=\mathcal{D}_{\Psi_{0}}^{*} g(\psi) .
$$

Claim The equation $L_{\Psi_{0}} \psi=f$ is uniquely solvable in $\operatorname{Ker} L_{\Psi_{0}}^{\frac{1}{\Psi_{0}} \cap H^{2} \text { for }}$ $f \in \operatorname{Ker} L_{\Psi_{0}}^{\perp}$.

Proof of Claim To see this let $G_{\Psi_{0}}: f \mapsto \psi$ be the associated Green operator. By Lax-Milgram this is a bounded linear operator from $\operatorname{Ker} L_{\Psi_{0}}^{\perp} \rightarrow$ $\operatorname{Ker} L_{\Psi_{0}}^{\perp} \cap H^{1}$. In fact since $\Psi_{0} \in W^{1, \infty}$ it follows from the formula for $L_{\Psi_{0}}$ in appendix $\mathrm{B}$ and elliptic regularity that it is a bounded linear operator $\operatorname{Ker} L_{\Psi_{0}}^{\perp} \rightarrow \operatorname{Ker} L_{\Psi_{0}}^{\perp} \cap H^{2}$.

Since $\mathcal{D}_{\Psi_{0}}^{*} g$ is automatically in $\operatorname{Ker} L_{\Psi_{0}}^{\perp}$ we can consider the function

$$
\begin{aligned}
Q: H^{2} & \rightarrow H^{2} \\
\psi & \mapsto \psi-G_{\Psi_{0}}\left(\mathcal{D}_{\Psi_{0}}^{*}(g(\psi)) .\right.
\end{aligned}
$$

Claim. $Q$ is a smooth function and has derivative equal to the identity at the origin.

Proof of Claim. This follows from the fact that the map

$$
\begin{aligned}
H^{2} & \rightarrow L^{2} \\
\psi & \mapsto \mathcal{D}_{\Psi_{0}}^{*} g(\psi)
\end{aligned}
$$

is smooth by the Sobolev theorems and the Green function is a bounded linear operator as just mentioned.

It follows that $Q$ is a diffeomorphism between neighbourhoods of the origin in $H^{2}$. Since $L_{\Psi_{0}(\epsilon t)} \psi=0$ if $\psi$ satisfies (5.25) all solutions of that equation are mapped by $Q$ into $\operatorname{Ker} L_{\Psi_{0}}$. This proves incidentally that if $O$ is a small neighbourhood of $\Psi_{0}$ in $\mathcal{A}^{(2)}$ then $\tilde{M}_{N}\left(\Psi_{0}\right) \bigcap O$ is a submanifold of $O$. We then define $\tilde{\Psi}_{0}(q)=\Psi_{0}+Q^{-1}(q)$.

Next we construct $G$. Recall that by corollary 5.8 we have a diffeomorphism $\tilde{G}^{-1}$ between a neighbourhood $V_{1}$ of $\pi\left(\Psi_{0}\right)$ in $\mathcal{M}^{(2)}$ and a neighbourhood $V_{2}$ of $\Psi_{0}$ in $\mathcal{S} \mathcal{L}_{\Psi_{0}}^{(2)}$. But by definition $\tilde{G}\left(M_{N} \cap V_{1}\right) \subset \tilde{M}_{N}\left(\Psi_{0}\right)$ and 
$\tilde{G}^{-1}\left(\tilde{M}_{N}\left(\Psi_{0}\right) \cap V_{2}\right) \subset M_{N}$. Therefore since $\tilde{M}_{N}\left(\Psi_{0}\right)$ is a submanifold it follows that $M_{N}$ is a submanifold of $\mathcal{M}^{(2)}$ and $\tilde{G}$ restricts to a diffeomorphism $G$ with the required properties. $F$ is defined by $F=\tilde{\Psi}_{0}^{-1} \circ G$. It follows from this construction that $G$ and $\pi$ are inverse to one another as stated; this implies the statement about $\pi_{*}$. The $W^{k, \infty}$ statement is proved in lemma B.3.

Remark. A choice of basis $e_{\mu}$ for the linear space $\operatorname{Ker} L_{\Psi_{0}}$ induces a coordinate system on $M_{N}$ around $\pi\left(\Psi_{0}\right)$ in which the point $q \in M_{N}$ is represented by an $2 N$-tuple $q_{\mu}$ with $F(q)=\sum q_{\mu} e_{\mu}$. Hereafter we will write $q$ both for the point in $M_{N}$ (or $\left.M_{N}^{(2)}\right)$ and for the $2 N$-tuple $\left(q_{\mu}\right)$. Locally points of the manifold $\tilde{M}_{N}\left(\Psi_{0}\right)$ can be written as $\tilde{\Psi}_{0}(q)$ for $q \in U_{2}$.

Theorem 5.12. (i) $S_{N}^{(2)} \subset \mathcal{A}^{(2)}$ is a submanifold with tangent and $L^{2}$ normal spaces at $\Psi_{0}$ given, respectively, by

$$
\begin{aligned}
& T\left(\Psi_{0}\right) \equiv \operatorname{Ker} L_{\Psi_{0}} \oplus T_{\Psi_{0}}\left(\mathcal{G}^{(3)} \cdot \Psi_{0}\right) \\
& N\left(\Psi_{0}\right) \equiv \mathcal{S} \mathcal{L}_{\Psi_{0}}^{(2)} \cap \operatorname{Ker} L_{\Psi_{0}}^{\perp}
\end{aligned}
$$

(ii) $\quad \pi: S_{N}^{(2)} \rightarrow M_{N}$ is a principal $\mathcal{G}^{(3)}$ bundle. The space $N\left(\Psi_{0}\right)$ is the $L^{2}$-orthogonal complement of $T\left(\Psi_{0}\right)$ in $T_{\Psi_{0}} \mathcal{A}^{(2)}$.

Remark. Assertion (i) above follows immediately from the decomposition in lemma 5.6 and the identification of the tangent space to $\tilde{M}_{N}$ with $\operatorname{Ker} L_{\Psi_{0}}$ (see the remark following definition 3). However, it is convenient for what follows to introduce the projections below (and reprove this statement).

Proof. (i) Define the Hilbert spaces $E_{1}=N\left(\Psi_{0}\right)$ and $E_{2}=T\left(\Psi_{0}\right)$ with $H^{2}$ norm. Let $\Pi_{\Psi_{0}}$ (resp. $\left.1-\Pi_{\Psi_{0}}\right)$ be the $L^{2}$-projection from $H^{2}\left(\mathbb{R}^{2} ; \mathbb{R}^{2} \times \mathbb{C}\right)$ to $E_{1}$ (resp. $E_{2}$ ). Recall from lemma 5.6 that $\Theta_{\Psi_{0}}$ varies continuously in operator norm. Also by lemma 5.11 there is a basis of $\operatorname{Ker} L_{\Psi_{0}}$ which varies smoothly in $H^{2}$ as a function of $\Psi_{0}$. Therefore the operators $\Pi_{\Psi_{0}},\left(1-\Pi_{\Psi_{0}}\right)$ are smooth in the operator norm with respect to $\Psi_{0} \in S_{N}^{(2)}$. It follows with the notation of lemma 5.11 and corollary 5.8 that the map

$$
\begin{aligned}
\mathcal{A}^{(2)} & \rightarrow E_{1} \times E_{2} \\
\Psi & \mapsto\left(\Pi_{\Psi_{0}} \circ Q \circ \Xi_{1},\left(\left(1-\Pi_{\Psi_{0}}\right) \circ Q \circ \Xi_{1}, \operatorname{Exp}^{-1} \circ \Xi_{2}\right)\right)
\end{aligned}
$$


is a diffeomorphism from a neighbourhood $V$ of $\Psi_{0} \in \mathcal{A}^{(2)}$ to a neighbourhood $V_{1} \times V_{2}$ of $(0,0)$ in $E_{1} \times E_{2}$ such that $V \cap S_{N}^{(2)}$ is mapped into $\{0\} \times V_{2}$. This proves that $S_{N}^{(2)}$ is a submanifold of $\mathcal{A}^{(2)}$.

(ii) $\mathcal{G}^{(3)}$ acts smoothly and freely on $S_{N}^{(2)}$ and $\pi$ is a smooth surjective submersion. Lemma 5.11 proves local triviality.

5.2.iii. The Ansatz. We now return to the solution of the time dependent equations. Let $\Psi(t)$ be the solution at time $t$ which, as stated in the beginning of section 5.b, is in the temporal gauge. For subsequent times it will be necessary to change gauges (so a time component to the connection will appear). We give an ansatz for the solutions of (2.1) and define the gauge in which we work. We have a small parameter $\epsilon$ which represents the distance of $\Psi(t)$ from the solution space $S_{N}^{(2)}$. It is convenient to introduce this explicitly into our ansatz; thus we will introduce a change of variable

$$
\tau(t)=\epsilon t .
$$

By a slight abuse of notation we will write functions $\tilde{f}(\tau)=f(\epsilon t)$ as $f(\tau)$ and we will write

$$
f^{\prime}=\frac{d}{d \tau} f \quad \dot{f}=\frac{d}{d t} f
$$

so that $\dot{f}=\epsilon f^{\prime}$. We will use the following convention: in equations in which some variables have argument $\tau$ and others have argument $t$ it is to be understood that $\tau=\epsilon$. Recall that $\Psi(t)$ is the solution in the temporal gauge. We will search for a gauge transformation $\chi(t)$ such that we can write the solution $\left(A_{0}^{\#}, \Psi^{\#}\right)=\rho(\chi(t))(0, \Psi)$ as

$$
\begin{aligned}
& \Psi^{\#}(t)=\Psi_{0}(\epsilon t)+\epsilon \psi(t)=(a(\epsilon t), \phi(\epsilon t))+\epsilon(\beta(t), \eta(t)) \\
& A_{0}^{\#}(t)=\epsilon^{2} \beta_{0}(t)=\frac{\partial \chi}{\partial t}
\end{aligned}
$$

where $A_{0}^{\#}$ is the time-component of the connection. Theorem 5.15 will allow us to interpret $\Psi_{0}(t)$ as the $L^{2}$ projection of $\Psi^{\sharp}(t)$ onto $S_{N}^{(2)}$. The terms $\beta_{0}$, $\beta$ and $\eta$ will be shown to vanish in $H^{2}$ norm as $t \rightarrow \infty$. In addition we will require, as gauge conditions, that

$$
\begin{aligned}
& \frac{d}{d \tau}(a(\tau), \phi(\tau))=\frac{d}{d \tau} \Psi_{0}(\tau) \in \mathcal{S} \mathcal{L}_{\Psi_{0}(\tau)} \\
& (\beta(t), \eta(t))=\psi(t) \in \mathcal{S} \mathcal{L}_{\Psi_{0}(\epsilon t)} .
\end{aligned}
$$


The possibility of applying these gauge transformations will follow from lemma 5.7 and the following result. This states that if we regard $S_{N}^{(2)}$ as a principal bundle over $M_{N}$ with fibre $\mathcal{G}^{(3)}$ then given a curve in moduli space $M_{N}$ it can be lifted horizontally to a curve in $S_{N}$ so that the vertical component of the velocity at each point is zero.

Lemma 5.13 (Existence of Horizontal Lift). Let there be given a continuously differentiable curve in the moduli space $\tau \mapsto q(\tau) \in M_{N}$ and $\Psi_{0}(0) \in S_{N}^{(2)} \bigcap_{k \in \mathbb{N}} W^{k, \infty}\left(\mathbb{R}^{2} ; \mathbb{R}^{2} \times \mathbb{C}\right)$ at $\tau=0$ such that $\pi\left(\Psi_{0}(0)\right)=$ $q(0)$. Then there exists a continuously differentiable curve $\tau \mapsto \Psi_{0}(\tau)=$ $(a(\tau), \phi(\tau)) \in S_{N}^{(2)}$ starting at $\Psi_{0}(0)$ and such that

$$
\begin{aligned}
& \pi\left(\Psi_{0}(\tau)\right)=q(\tau) \\
& \frac{d}{d \tau}(a(\tau), \phi(\tau)) \in \mathcal{S} \mathcal{L}_{a(\tau), \phi(\tau)}^{(2)} .
\end{aligned}
$$

The function $\tau \mapsto\left(\Psi_{0}(\tau)-\Psi_{0}(0)\right) \in H^{2}\left(\left(\mathbb{R}^{2} ; \mathbb{R}^{2} \times \mathbb{C}\right)\right)$ is differentiable. Furthermore, for all $K$ sufficiently small there exists a number $c(n, K)$ such that

$$
\int_{0}^{S}\left|q^{\prime}(\tau)\right| d \tau \leq K
$$

implies

$$
\max _{\tau \in[0, S]}\left(\left\|\Psi_{0}(\tau)-\Psi_{0}(0)\right\|_{W^{n, \infty}}+\left\|\Psi_{0}(\tau)-\Psi_{0}(0)\right\|_{H^{n+2}}\right) \leq c(n, K)\|q\|_{C^{1}([0, S])} .
$$

Finally let $q(\tau), q^{*}(\tau)$ be two $C^{1}$ curves starting at $\pi\left(\Psi_{0}(0)\right)$ and satisfying (5.34) and let $\Psi_{0}(\tau), \Psi_{0}^{*}(\tau)$ be their respective lifts. Then there exist constants $c_{1}, c_{2}$ such that if $\|q\|_{C^{1}([0, S])}+\left\|q^{*}\right\|_{C^{1}([0, S])}<c_{2}$ then

$$
\max _{0 \leq \tau \leq S}\left\|\Psi_{0}(\tau)-\Psi_{0}^{*}(\tau)\right\|_{H^{2}} \leq c_{1} S\left\|q-q^{*}\right\|_{C^{1}([0, S])}
$$

Proof of lemma 5.13 Assume without loss of generality that $q(0)=0$ (and recall the remark above theorem 5.12 regarding the notation of points in $M_{N}$ ). We will use the diffeomorphism $\tilde{\Psi}_{0}$ provided by lemma 5.11 (with $\left.\Psi_{0}=\Psi_{0}(0)\right)$ to obtain a curve $\tau \mapsto \tilde{\Psi}_{0}(q(\tau)) \in \tilde{M}_{N}\left(\Psi_{0}\right)$. We now look for $\tau \mapsto \chi(\tau) \in \mathcal{G}^{(3)}$ such that $\tau \mapsto \Psi_{0}(\tau)=\rho(\chi) \tilde{\Psi}_{0}(q(\tau))$ satisfies the required condition. Differentiating we get (using the notation defined in (5.29))

$$
\frac{d}{d \tau} \Psi_{0}(\tau)=\left(\sum_{\mu=1}^{2 N} q_{\mu}^{\prime} \frac{\partial \tilde{a}}{\partial q_{\mu}}+\nabla \chi^{\prime}, e^{i \chi}\left(\sum_{\mu=1}^{2 N} q_{\mu}^{\prime} \frac{\partial \tilde{\phi}}{\partial q_{\mu}}+i \chi^{\prime} \tilde{\phi}\right)\right)
$$


so that $\chi^{\prime}$ must solve the equation for each $\tau$

$$
-\Delta \chi^{\prime}+|\tilde{\phi}(q(\tau))|^{2} \chi^{\prime}=\nabla \cdot\left(\sum_{\mu=1}^{2 N} q_{\mu}^{\prime} \frac{\partial \tilde{a}}{\partial q_{\mu}}\right)+\left(i \tilde{\phi}, \sum_{\mu=1}^{2 N} q_{\mu}^{\prime} \frac{\partial \tilde{\phi}}{\partial q_{\mu}}\right) \in L^{2} .
$$

This is solvable since the operator on the left is an isomorphism from $H^{3} \rightarrow H^{1}$ for each $\tau$ (lemma B.2). The differentiability statements follow from the elliptic estimates. Let $K$ be small enough that (5.34) ensures $q$ remains within the same coordinate chart. Lemma B.3 then implies that $\left\|\chi^{\prime}(\cdot, \tau)\right\|_{H^{n}} \leq c(n, K)\|q\|_{C^{1}}$ for all $n$. This implies the bound (5.35). Finally to prove the continuity of the lifting, let $c_{2}$ be small enough that $q, q^{*}$ both lie in the same coordinate chart and let $\chi^{\prime},\left(\chi^{*}\right)^{\prime}$ be the solutions of (5.36) and the corresponding equation for $q^{*}$. Then by subtracting the equations and applying the elliptic regularity estimate it follows that $\left\|\chi^{\prime}-\left(\chi^{*}\right)^{\prime}\right\|_{H^{3}} \leq c\left\|q-q^{*}\right\|_{C^{1}[0, S]}$ and the result follows by integration.

Remark. [Definition of $n_{\mu}$ ]. Let $\left\{e_{\mu}\right\}_{\mu=1}^{2 N}$ be a basis for $T_{\Psi_{0}(0)} \tilde{M}_{N}=\operatorname{Ker} L_{\Psi_{0}(0)}$ so that a point $q$ is given as $q=\sum_{\mu} q_{\mu} e_{\mu}$. Considering a local family of solutions $q \mapsto \tilde{\Psi}_{0}(q)$ as in lemma 5.11 note that $u_{\mu}=\frac{\partial \tilde{\Psi}_{0}}{\partial q_{\mu}}(q) \in T_{\tilde{\Psi}_{0}(q)} \tilde{M}_{N}$ solves the linearised Bogomol'nyi equations. Now recall that $\operatorname{Ker} L_{\tilde{\Psi}_{0}(q)}$ can be identified with $\operatorname{Ker} \mathcal{D}_{\tilde{\Psi}_{0}(q)}$, the operator described above and in appendix B. It follows from this description that the $L^{2}$-projection operator defined in lemma $5.6 \Theta_{\tilde{\Psi}_{0}(q)}$ restricted to $T_{\tilde{\Psi}_{0}(q)} \tilde{M}_{N}$ maps into $\operatorname{Ker} L_{\tilde{\Psi}_{0}(q)}$. We may thus display a basis for $T_{\tilde{\Psi}_{0}(q)} \tilde{M}_{N}$ by forming for $\mu=1, \ldots, 2 N$

$$
\tilde{n}_{\mu}(q)=\Theta_{\tilde{\Psi}_{0}(q)}\left(\frac{\partial \tilde{\Psi}_{0}}{\partial q_{\mu}}\right)(q) .
$$

The $\tilde{n}_{\mu}(q)$ vary smoothly with respect to $q$ in the $H^{2}$ norm by lemmas 5.6 and 5.11. Under a gauge transformation $\tilde{\Psi}_{0} \rightarrow \rho(\chi) \tilde{\Psi}_{0}$ we have $\tilde{n}_{\mu} \rightarrow(\rho(\chi))_{*} \tilde{n}_{\mu}$ where $\rho(\chi)_{*}(\beta, \eta)=\left(\beta, \eta e^{i \chi}\right)$. Let $q(\tau)$ and $\Psi_{0}(\tau)$ be curves as in lemma 5.13. It follows that we can write

$$
\frac{d}{d \tau} \Psi_{0}(\tau)=\sum_{\mu=1}^{2 N} q_{\mu}^{\prime}(\tau) n_{\mu}(\tau)
$$

where $n_{\mu}(\tau)=\rho(\chi(\tau))_{*}\left(\tilde{n}_{\mu}(q(\tau))\right)$. From this we see that $L_{\Psi_{0}(\tau)} n_{\mu}(\tau)=0$ and for sufficiently small $\tau$ the $\left\{n_{\mu}(\tau)\right\}_{\mu=1}^{2 N}$ form a basis for $\operatorname{Ker} L_{\Psi_{0}(\tau)}$ since 
linear independence is an open condition. Furthermore it follows from this definition and the proof of lemma 5.13 that

$$
\max _{0 \leq \tau \leq S}\left\|n_{\mu}(\tau)\right\|_{H^{n}} \leq c(n, K)\|q\|_{C^{1}([0, S])}
$$

Let $n_{\mu}^{*}(\tau)$ be defined in the same way for a curve $q^{*}(\tau)$ as in lemma 5.13, then by the proof of lemma 5.13 for $\mu=1, \ldots, 2 N$ we have

$$
\begin{aligned}
\max _{0 \leq \tau \leq S}\left\|n_{\mu}^{*}(\tau)-n_{\mu}(\tau)\right\|_{H^{2}} \leq c(K) S\left\|q-q^{*}\right\|_{C^{1}([0, S])} \\
\max _{0 \leq \tau \leq S}\left\|\frac{d n_{\mu}^{*}}{d \tau}(\tau)-\frac{d n_{\mu}}{d \tau}(\tau)\right\|_{H^{2}} \leq c(K)\left\|q-q^{*}\right\|_{C^{1}([0, S])}
\end{aligned}
$$

Next we prove theorem 5.15, a result on the existence of tubular neighbourhoods. As remarked previously this is nonstandard in that $S_{N}^{(2)}$ is a submanifold of $\mathcal{A}^{(2)}$, which has an $H^{2}$ topology whereas our tubular neighbourhood will be constructed with respect to $L^{2}$. The possibility of doing this follows from the following regularity result. In the next two results $\Psi_{*}$ is as in theorem 5.4 .

Lemma 5.14 (Regularity). Assume $F \in S_{N}^{(2)}$ and $(F-\Psi) \in \mathcal{S L}_{\Psi}^{(2)}$. Then there exists a number $c=c\left(\Psi_{*}\right)$ such that

$$
\left\|F-\Psi_{*}\right\|_{H^{2}} \leq c\left(\left\|F-\Psi_{*}\right\|_{L^{2}}+\left\|\Psi-\Psi_{*}\right\|_{H^{2}}\right)
$$

Proof. This is proved in appendix C.

Theorem 5.15 (Tubular Neighbourhood). There exist numbers $L_{3}>$ $0, \theta>0$ depending only on $\Psi_{*}$ and a smooth function $\mathbb{P}: B_{\theta} \rightarrow S_{N}^{(2)}$ defined on the ball

$$
B_{\theta} \equiv\left\{\Psi \in \mathcal{A}^{(2)}:\left\|\Psi-\Psi_{*}\right\|_{H^{2}}<\theta\right\}
$$

such that for all $\Psi \in B_{\theta}$ the point $\mathbb{P}(\Psi)$ is the unique point of $S_{N}^{(2)}$ such that either of the following two conditions hold

(i) $\quad\|\Psi-\mathbb{P}(\Psi)\|_{L^{2}}=\inf _{P \in S_{N}^{(2)}}\|\Psi-P\|_{L^{2}}$, or

(ii) $\quad(\Psi-\mathbb{P}(\Psi)) \in \mathcal{S} \mathcal{L}_{\mathbb{P}(\Psi)}^{(2)} \cap K \operatorname{Ker} L_{\mathbb{P}(\Psi)}^{\perp}$ and $\|\Psi-\mathbb{P}(\Psi)\|_{H^{2}}<L_{3} \theta$.

In addition $\mathbb{P}(\Psi)$ has the following properties

(iii) if $\Psi \in \bigcap_{k \in \mathbb{N}} W^{k, \infty}\left(\mathbb{R}^{2} ; \mathbb{R}^{2} \times \mathbb{C}\right)$ then $\mathbb{P}(\Psi) \in \bigcap_{k \in \mathbb{N}} W^{k, \infty}\left(\mathbb{R}^{2} ; \mathbb{R}^{2} \times \mathbb{C}\right)$

(iv) if $g \in \mathcal{G}^{(3)}$ then $\mathbb{P}(\rho(g) \Psi)=\rho(g) \mathbb{P}(\Psi)$. 
Proof. Step One. The $L^{2}$-normal space $N\left(\Psi_{0}\right)$ to $S_{N}^{(2)}$ at $\Psi_{0}$ was defined in (5.27). It is endowed with the $H^{2}$ norm. Let $\Pi_{\Psi_{0}}: N\left(\Psi_{*}\right) \rightarrow N\left(\Psi_{0}\right)$ be the restriction of the $L^{2}$-projection onto $N\left(\Psi_{0}\right)$. Notice that $\Pi_{\Psi_{0}}$ is continuous in the operator topology (induced from $H^{2}$ ) with respect to $\Psi_{0} \in S_{N}^{(2)}$ (see the proof of theorem 5.12). Results of Kato (see [21, 20]) imply the following:

Claim For $\left\|\Psi_{0}-\Psi_{*}\right\|_{H^{2}}$ small $\Pi_{\Psi_{0}}$ is a bounded linear bijection from $N\left(\Psi_{*}\right)$ onto $N\left(\Psi_{0}\right)$.

(This can also be proved directly with an implicit function theorem argument.) Now consider the map

$$
\begin{aligned}
I: U \times V & \rightarrow W \\
\left(\Psi_{0}, n\right) & \mapsto \Psi_{0}+\Pi_{\Psi_{0}} n .
\end{aligned}
$$

Here $U \times V$ is a neighbourhood of $\left(\Psi_{*}, 0\right)$ in $S_{N}^{(2)} \times N\left(\Psi_{*}\right)$ and $W$ is a neighbourhood of $\Psi_{*} \in \mathcal{A}^{(2)}$. The derivative of this map at $\left(\Psi_{*}, 0\right)$ is the linear map

$$
\begin{aligned}
T\left(\Psi_{*}\right) \times N\left(\Psi_{*}\right) & \rightarrow T_{\Psi_{*}} \mathcal{A}^{(2)} \\
(\psi, n) & \mapsto \psi+n .
\end{aligned}
$$

This is a bounded linear bijection. Therefore by the inverse function theorem we can choose $U, V, W$ small so that $I$ is a diffeomorphism. Write the inverse diffeomorphism as $I^{-1}(\Psi)=(\mathbb{P}(\Psi), \nu(\Psi))$. This allows us to write each point $\Psi \in W$ uniquely as $\Psi=\mathbb{P}(\Psi)+\Pi_{\mathbb{P}(\Psi)}(\nu(\Psi))$ with $\mathbb{P}(\Psi) \in U, \nu(\Psi) \in V$. The fact that $\Psi-\mathbb{P}(\Psi)$ satisfies condition (ii) isthen immediate.

Step Two. We next show that if $\Psi$ is close enough to $S_{N}^{(2)}$ then the minimisation in (i) in the theorem is attained. Thus there is a closest point on $S_{N}^{(2)}$ and it satisfies (ii) in the theorem. This is done in two stages. Firstly we show that the closest point on each orbit lies on the slice $\Psi+\mathcal{S} \mathcal{L}_{\Psi}^{(2)}$. We then show that there exists a point on $\left(\Psi+\mathcal{S} \mathcal{L}_{\Psi}^{(2)}\right) \cap S_{N}^{(2)}$ which is closest to $\Psi$.

Lemma 5.16. For $\Psi$ and $\Psi_{0}$ in $\mathcal{A}^{(2)}$ there exists $\chi \in \mathcal{G}^{(3)}$ such that

$$
\left\|\rho(\chi) \Psi_{0}-\Psi\right\|_{L^{2}}=\inf _{\zeta \in \mathcal{G}^{(3)}}\left\|\rho(\zeta) \Psi_{0}-\Psi\right\|_{L^{2}}
$$

and $\chi$ is such that $\rho(\chi) \Psi_{0}-\Psi \in \mathcal{S L}_{\Psi}^{(2)}$. Also if both $\Psi$ and $\Psi_{0}$ lie in $\bigcap_{k \in \mathbb{N}} W^{k, \infty}\left(\mathbb{R}^{2} ; \mathbb{R}^{2} \times \mathbb{C}\right)$ then so does $\rho(\chi) \Psi_{0}$. 
Proof. The proof of this is in appendix C.

Lemma 5.17. There exist numbers $L_{3}>1, \epsilon_{3}>0$ such that if $\epsilon<\epsilon_{3}$ the following is true. Let $\Psi=(A, \Phi) \in \bigcap_{k \in \mathbb{N}} W^{k, \infty}\left(\mathbb{R}^{2} ; \mathbb{R}^{2} \times \mathbb{C}\right)$ satisfy $\left\|\Psi-\Psi_{*}\right\|_{H^{2}}<\epsilon$. Then there exists $\Psi_{0} \in S_{N}^{(2)} \bigcap_{k \in \mathbb{N}} W^{k, \infty}\left(\mathbb{R}^{2} ; \mathbb{R}^{2} \times \mathbb{C}\right)$ such that $\left(\Psi-\Psi_{0}\right) \in \mathcal{S} \mathcal{L}_{\Psi_{0}}^{(2)} \cap \operatorname{Ker} L_{\Psi_{0}}^{\perp},\left\|\Psi-\Psi_{0}\right\|_{H^{2}}<L_{3} \epsilon$ and

$$
\left\|\Psi-\Psi_{0}\right\|_{L^{2}}=\inf _{P \in S_{N}^{(2)} \cap\left(\Psi+\mathcal{S} \mathcal{L}_{\Psi}^{(2)}\right)}\|P-\Psi\|_{L^{2}}
$$

Proof. The proof of this is in appendix C.

STep Three. We now know that there is an $L^{2}$-closest point to $\Psi$ on $S_{N}^{(2)}$. We must show that if $\theta$ is small enough it is unique. So assume to the contrary that for all $\theta>0$ it is not true that if $\Psi \in B_{\theta}$ then $\mathbb{P}(\Psi)$ is the unique point which satisfies (i) in theorem 5.15. In this case there exists a sequence of points $\left\{\Psi_{i}\right\}_{i=1}^{\infty}$ such that

(i) $\lim _{i \rightarrow \infty}\left\|\Psi_{i}-\Psi_{*}\right\|_{H^{2}}=0$

(ii) $\Psi_{i}=P_{i}+n_{i}$ with $P_{i} \neq \mathbb{P}\left(\Psi_{i}\right)$ and $\left\|\Psi_{i}-P_{i}\right\|_{L^{2}}=\left\|\Psi_{i}-\mathbb{P}\left(\Psi_{i}\right)\right\|_{L^{2}}$.

The second condition implies $n_{i} \in N\left(P_{i}\right)$ by the Euler-Lagrange equation. This in turn implies $n_{i}=\left(\Psi_{i}-P_{i}\right) \in \mathcal{S L}_{\Psi_{i}}^{(2)}$ by the remark following definition 1. Therefore by lemma 5.14 we have

$$
\left\|P_{i}-\Psi_{*}\right\|_{H^{2}} \leq c\left(\left\|\Psi_{i}-\Psi_{*}\right\|_{H^{2}}+\left\|P_{i}-\Psi_{*}\right\|_{L^{2}}\right)
$$

But

$$
\begin{aligned}
\left\|P_{i}-\Psi_{*}\right\|_{L^{2}} & \leq\left\|\Psi_{i}-P_{i}\right\|_{L^{2}}+\left\|\Psi_{i}-\Psi_{*}\right\|_{L^{2}} \\
& \leq\left\|\Psi_{i}-\mathbb{P}\left(\Psi_{i}\right)\right\|_{L^{2}}+\left\|\Psi_{i}-\Psi_{*}\right\|_{L^{2}}
\end{aligned}
$$

and therefore

$$
\lim _{i \rightarrow+\infty}\left(\left\|P_{i}-\Psi_{*}\right\|_{H^{2}}+\left\|\Psi_{i}-P_{i}\right\|_{H^{2}}+\left\|\Psi_{i}-\Psi_{*}\right\|_{H^{2}}\right)=0
$$

Now define $\nu_{i}$ to be the unique element of $N\left(\Psi_{*}\right)$ such that $\Pi_{P_{i}}\left(\nu_{i}\right)=n_{i}$. Such a $\nu_{i}$ exists and is unique for $\left\|P_{i}-\Psi_{*}\right\|_{H^{2}}$ sufficiently small since $\Pi_{P_{i}}$ : $N\left(\Psi_{*}\right) \rightarrow N\left(P_{i}\right)$ is a bounded linear bijection by step one. Furthermore the $\nu_{i}$ will satisfy $\lim _{i \rightarrow \infty}\left\|\nu_{i}\right\|_{H^{2}}=0$ since

$$
\left\|\nu_{i}\right\|_{H^{2}} \leq\left\|n_{i}\right\|_{H^{2}}+\left\|\left(\Pi_{P_{i}}-\Pi_{\Psi_{*}}\right) \nu_{i}\right\|_{H^{2}}
$$


This now gives the desired contradiction; if we take $i$ large we may assume $\Psi_{i} \in W, \nu_{i} \in V, P_{i} \in U$. Therefore by the uniqueness in step one $P_{i}=\mathbb{P}\left(\Psi_{i}\right)$ contrary to assumption.

5.2.iv. Proof of the Adiabatic Approximation theorem. Assume $\Psi(t)$ and $\Psi_{*}$ are as in the theorem with $\left\|\Psi_{*}-\Psi(0)\right\|_{H^{2}}<\epsilon$ which will be specified later. As explained, we show that the projection $\mathbb{P}(\Psi(t))$ of $\Psi(t)$ on $S_{N}^{(2)}$ satisfies the theorem (where $\mathbb{P}$ is defined in theorem 5.15); in particular, its $H^{2}$-distance from $\Psi(t)$ asymptotically vanishes and the two curves converge to a unique limit $\Psi_{\infty} \in S_{N}^{(2)}$. To achieve this we determine $\Psi^{\#}(t)$ a gauge equivalent curve to $\Psi(t)$ which appears in our ansatz for the solution given in (5.30-5.31) together with gauge conditions (5.32-5.33). For initial conditions we have $\Psi^{\#}(0)=\Psi(0)$. We will prove that the solution can be written in this way for all times and $\lim _{t \rightarrow+\infty}\|\psi(t)\|_{H^{2}}=0$. (We will see later that $\left.\Psi_{0}(\epsilon t)=\mathbb{P}\left(\Psi^{\#}(t)\right)\right)$. First we determine an initial point $\Psi_{0}(0) \in S_{N}^{(2)}$ in step one. Assuming that a curve $q(\epsilon t)$ is given in $M_{N}$ we determine $\Psi^{\#}(t)$ and obtain the equations satisfied for the error term $\psi(t)$ in step two. In step three we obtain the curve $q$ and in step four we show that the Hessian can be used as a Liapunov functional to show the exponential convergence to a limit. This is concluded in step five.

Step One. Determination of $\Psi_{0}(0)$ Initially we take $\epsilon<\min \left\{\theta, \epsilon_{2} / L_{3}\right\}$ to allow application of lemmas 5.7-5.9 on some small time interval to fix the gauge. We will make $\epsilon$ sucessively smaller as the proof progresses. We determine $\Psi_{0}(0)$ as the $L^{2}$-projection of $\Psi(0)$ onto $S_{N}^{(2)}$ : apply theorem 5.15 with $\Psi=\Psi(0)$ and $\Psi_{*}$ as in the statement of theorem 5.4. This provides us with $\Psi_{0}(0)=\mathbb{P}(\Psi(0)) \in S_{N}^{(2)} \bigcap_{k \in \mathbb{N}} W^{k, \infty}\left(\mathbb{R}^{2} ; \mathbb{R}^{2} \times \mathbb{C}\right)$ satisfying

$$
\begin{aligned}
& \left\|\Psi(0)-\Psi_{0}(0)\right\|_{H^{2}} \leq L_{3} \epsilon \\
& \Psi(0)-\Psi_{0}(0) \in\left(\mathcal{S L}_{\Psi_{0}(0)}^{(2)}\right) \bigcap \operatorname{Ker} L_{\Psi_{0}(0)}^{\perp}
\end{aligned}
$$

Step Two. Choice of Gauge, $\Psi^{\#}(t)$ and $\psi(t)$ Throughout this step we assume that the curve $q(\epsilon t)=\pi\left(\Psi_{0}(\epsilon t)\right.$ ) is given in $M_{N}$ (starting at $\pi\left(\Psi_{0}\right)$ ) although we will not specify it until step three. Using the local diffeomorphism $F$ in lemma 5.11 we slightly abuse notation and use $q=$ $\left\{q_{\mu}\right\}_{\mu=1}^{2 N}$ to designate both points in a coordinate chart of $\pi\left(\Psi_{0}(0)\right)$ and points of $\operatorname{Ker} L_{\Psi_{0}}$ (as explained in the remark following the same lemma). So let $q(\epsilon t)=q(\tau)$ be any $C^{1}$ curve in the chart in $M_{N}$ with $\pi\left(\Psi_{0}\right)=q(0)=0$. We apply the map $\tilde{\Psi}_{0}$ of lemma 5.11 to obtain a local family of solutions 
$q \rightarrow \tilde{\Psi}_{0}(q) \in \tilde{M}_{N}\left(\Psi_{0}\right) . \quad \tilde{\Psi}_{0}(q)$ varies smoothly in the $H^{2}$ topology with respect to $q$ and $\tilde{\Psi}_{0}(q) \in \bigcap_{k \in \mathbb{N}} W^{k, \infty}\left(\mathbb{R}^{2} ; \mathbb{R}^{2} \times \mathbb{C}\right)$. We remind the reader of our convention regarding the slow time variable $\tau$ defined in (5.28). By lemma 5.13 the curve $\tilde{\Psi}_{0}(q(\tau)) \in \tilde{M}_{N}\left(\Psi_{0}\right)$ is lifted horizontally to the curve $\Psi_{0}(\tau) \in S_{N}^{(2)}$ such that $\tau \mapsto \Psi_{0}(\tau)$ is differentiable into the $H^{2}$ topology and for each $\tau$ satisfies $\Psi_{0}(\tau) \in \bigcap_{k \in \mathbb{N}} W^{k, \infty}\left(\mathbb{R}^{2} ; \mathbb{R}^{2} \times \mathbb{C}\right) \cap S_{N}^{(2)} . \operatorname{Next}(5.43)$ implies by continuity $\left\|\Psi(t)-\Psi_{0}(\epsilon t)\right\|_{H^{2}}<L_{3} \epsilon$ for small $\tau$. Since $L_{3} \epsilon<\epsilon_{1}$ lemma 5.7 applies to provide a gauge transformation $\chi(\tau)$ such that

$$
\Psi^{\#}(t)-\Psi_{0}(\epsilon t)=\left(\rho(\chi(t)) \Psi(t)-\Psi_{0}(\epsilon t)\right) \in \mathcal{S} \mathcal{L}_{\Psi_{0}(\epsilon t)}^{(2)}
$$

The lifted curve $\Psi_{0}(\cdot)$ as well as the gauge transformation $\chi(\cdot)$ depend on the curve $q(\cdot)$ and to this effect we will insert this dependence in the notation $\Psi_{0}(\tau ; q)$ and $\chi(t ; q)$ respectively when it is necessary (lemma 5.19). The dependence of $\Psi_{0}$ and $\chi$ on $q$ will be supressed when no confusion is possible.

Now we determine the equation satisfied by $\psi(t)$. Substitute the ansatz (5.30)-(5.31), with these gauge conditions, into equations (2.1). This leads to the following set of equations (where we show explicitly the dependence on the scaled time variable $\tau=\epsilon t$ suppressing dependence on $t$ )

$$
\dot{\beta}+\left(-\Delta+|\phi(\tau)|^{2}\right) \beta+2\left(i D_{a(\tau)} \phi(\tau), \eta\right)=-\frac{d}{d \tau} a(\tau)+\epsilon f\left(\Psi_{0}(\tau), \beta, \eta, \beta_{0}\right)
$$

$$
\begin{aligned}
\dot{\eta}+\left(-\Delta_{a(\tau)}+|\phi(\tau)|^{2}\right) \eta+2 i \beta \cdot D_{a(\tau)} \phi(\tau) & -\frac{1}{2}\left(1-|\phi|^{2}(\tau)\right) \eta= \\
& -\frac{d}{d \tau} \phi(\tau)+\epsilon g\left(\Psi_{0}(\tau), \beta, \eta, \beta_{0}\right)
\end{aligned}
$$

$$
\left(-\Delta+|\phi(\tau)|^{2}\right) \beta_{0}=h\left(\Psi_{0}(\tau), \eta, \dot{\eta}\right)
$$

where $\Psi_{0}(\tau ; q)=(a(\tau), \phi(\tau))$. The nonlinear error terms $f, g, h$ are given by:

$$
\begin{aligned}
f & =\nabla \beta_{0}-2(\phi, \eta) \beta+\left(i \eta, D_{a} \eta\right)-\epsilon|\eta|^{2} \beta \\
g & =\left(i \beta_{0}+|\beta|^{2}\right)(\phi+\epsilon \eta)+2 i \beta \cdot D_{a} \eta+i(\nabla \cdot \beta) \eta-\eta(\phi, \eta)-\frac{1}{2}|\eta|^{2} \phi-\frac{1}{2} \epsilon|\eta|^{2} \eta \\
h & =\left(2 i \eta, \frac{d \phi}{d \tau}\right)+\left(i \eta, \frac{d \eta}{d t}\right) .
\end{aligned}
$$


Equations (5.45-5.46) can be written in the form

$$
\dot{\psi}+L_{\Psi_{0}(\tau)} \psi=-\frac{d}{d \tau} \Psi_{0}(\tau)+\epsilon j\left(\Psi_{0}(\tau), \beta, \eta, \beta_{0}\right)
$$

where the operator $L_{\Psi_{0}}$ is defined in (5.22) and appendix B. We will also use the differentiated version of this equation

$$
\mathrm{D}_{\mathrm{a}} \dot{\psi}+L_{\Psi_{0}(\tau)}\left(\mathrm{D}_{\mathrm{a}} \psi\right)+\left[\mathrm{D}_{\mathrm{a}}, L_{\Psi_{0}(\tau)}\right] \psi=-\mathrm{D}_{\mathrm{a}}\left(\frac{d}{d \tau} \Psi_{0}(\tau)\right)+\epsilon \mathrm{D}_{\mathrm{a}} j\left(\Psi_{0}(\tau), \beta, \eta, \beta_{0}\right)
$$

where $D_{a}$ is as defined in appendix $A$.

Step Three. Determination of $q(\epsilon t)$ The next stage is to construct the curve $q(\epsilon t)$ which represents a projection of $\Psi(t)$ onto the moduli space $M_{N}$. We will first write down an equation for $\dot{q}$ which ensures that the orthogonality condition in (5.44) holds for positive $t$. We will then show that this equation has a solution by the contraction mapping theorem. From step one we have a point $\Psi_{0}(0) \in S_{N} \cap S_{N}^{(2)}$ such that $\Psi(0)-\Psi_{0}(0)$ is orthogonal to $\operatorname{Ker} L_{\Psi_{0}}$ and gauge orthogonal with respect to $\Psi_{0}$ (see (5.44)). We want to define $q(\tau)$ in such a way that these orthogonality conditions persist for $t>0$; thus referring to $(5.30-5.31)$ we require that

$$
\left(\psi(t), n_{\mu}(\tau)\right)_{L^{2}}=0
$$

for all $n_{\mu}(\tau)$ as defined in (5.37). This requirement leads to the differential equation (5.50) in the next lemma. First, introduce a metric $g_{\mu \nu}$ on the moduli space $M_{N}$ by the formula

$$
g_{\mu \nu}(q) \equiv\left(\tilde{n}_{\mu}(q), \tilde{n}_{\nu}(q)\right)_{L^{2}} .
$$

Since $g$ is gauge invariant it defines a metric on $M_{N}$. (This has been studied in detail $([34,3])$ and is known to be a Kähler metric). Notice that the definition of $n_{\mu}(\tau)$ implies

$$
g_{\mu \nu}(q(\tau))=\left(n_{\mu}(\tau), n_{\nu}(\tau)\right)_{L^{2}} .
$$

Lemma 5.18 (Determination of $q(\tau)$ ). Assume that $q(\tau)$ is the solution of

$$
\begin{aligned}
& g_{\mu \nu}(q(\tau)) q_{\nu}^{\prime}(\tau)=\epsilon\left(\psi, \frac{d}{d \tau} n_{\mu}(\tau)\right)_{L^{2}}+\epsilon\left(n_{\mu}(\tau), j\right)_{L^{2}} \\
& q(0)=0 .
\end{aligned}
$$


Then

$$
(\psi(t), n)_{L^{2}}=0
$$

for all $n \in T_{\Psi_{0}(\epsilon t)} \tilde{M}_{N}$, if this is true at $t=0$.

Proof. Adopt the local coordinate system from lemma 5.11 and differentiate (5.51) using (5.48) to show that this derivative is zero if and only if $q$ satisfies (5.50). By assumption (5.51) is valid at $t=0$ and so it will be valid for all $t>0$.

Lemma 5.19 (Existence of $q(\tau)$ ). There exists a time $S>0$ and $\epsilon_{4}>0$ such that for $\epsilon<\epsilon_{4}$ there exists a $C^{1}$ solution $q(\tau)$ to $(5.50)$ on $[0, S]$ which satisfies (5.34).

Proof. $\Psi(t)$ is known and we wish to determine $q(\tau)$. We shall apply the contraction mapping theorem on $C^{1}\left([0, S] ; M_{N}\right)$. So assume $q, q^{*} \in$ $\left.C^{1}\left([0, S] ; M_{N}\right)\right)$ and let $\Psi_{0}(\tau), \Psi_{0}^{*}(\tau)$ be the respective horizontal lifts. As in step two gauge transformations $\chi(t), \chi^{*}(t)$ exist to make $\psi=\rho(\chi(t)) \Psi(t)-$ $\Psi_{0}(\epsilon t)$ and $\psi^{*}=\rho\left(\chi^{*}(t)\right) \Psi(t)-\Psi_{0}^{*}(\epsilon t)$ gauge orthogonal with respect to $\Psi_{0}(\epsilon t), \Psi_{0}^{*}(\epsilon t)$. Also it follows by (5.35) and lemma 5.9 that $\psi$ varies continuously in $H^{2}$ as a function of $q$ (note that $\chi$ in lemma 5.9 is estimable by $q$ through its smooth dependence on $\Psi_{0}$ ):

$$
\left\|\psi-\psi^{*}\right\|_{H^{2}} \leq c S\left\|q-q^{*}\right\|_{C^{1}\left([0, S] ; M_{N}\right)} .
$$

Next substitute for $\psi=\rho(\chi) \Psi-\Psi_{0}$ into the right hand side of (5.50). Using the uniform invertibility of the matrix $\left(g_{\mu \nu}\right)$ on the coordinate chart this gives an equation of the form

$$
q^{\prime}(\tau)=J\left(q, q^{\prime}\right) .
$$

It follows from lemmas 5.13 and 5.9 and (5.39- 5.40) that $J$ satisfies

$$
\max _{0 \leq \tau \leq S}\left|J(q)-J\left(q^{*}\right)\right| \leq c(S+\epsilon)\left\|q-q^{*}\right\|_{C^{1}\left([0, S] ; M_{N}\right)}
$$

for times $S$ short enough that $\epsilon\|\psi(S)\|_{H^{2}\left(\mathbb{R}^{2}\right)}<\min \left\{\epsilon_{1}, \epsilon_{2}\right\}$. (Note that since $\chi$ in lemma 5.9 is estimable in $q$ in $H^{3}$ via its dependence on $\Psi_{0}$, the terms in $J$ involving $\beta_{0}$ and $\nabla \beta_{0}$ are similarly estimable in $H^{2}$ ). Therefore for $\epsilon, S$ small enough we get a contraction in $C^{1}\left([0, S] ; M_{N}\right)$. This gives a solution 
which we call $q(\tau)$; by making $S$ sufficiently small we can ensure it satisfies (5.34).

It will follow from the analysis that this solution $q(\tau)$ can be extended indefinitely to a solution which satisfies (5.34) with $S=+\infty$ if $\epsilon$ is sufficiently small. Having thus determined $q(\tau)$ we apply lemma 5.13 to define $\Psi_{0}(\tau)$ as the horizontal lift of $q(\tau)$ with initial point $\Psi_{0}(0)$. For as long as $\| \Psi^{\#}(t)-$ $\Psi_{*} \|_{H^{2}}<\theta$ we know by theorem 5.15 that $\Psi_{0}(\epsilon t)=\mathbb{P}\left(\Psi^{\#}(t)\right)$.

Step Four. The Hessian as Liapunov Functional We will now show that the Hessian of $\mathcal{E}$ provides a good norm with which to estimate the solution and then obtain exponential decay in time of a functional $Q(t)$ built out of the Hessian evaluated at $\psi(t) . Q(t)$ is equivalent to the $H^{2}$ norm of $\psi$.

Lemma 5.20. Assume $q(\tau)=\pi\left(\Psi_{0}(\tau)\right)$ satisfies (5.34). Then there exists a positive number $\gamma$ such that for $0 \leq \tau \leq S$ :

$\gamma^{-1}\|\psi\|_{H^{2}\left(\mathbb{R}^{2}\right)}^{2} \leq\left(\psi, L_{\Psi_{0}(\tau)} \psi\right)_{L^{2}\left(\mathbb{R}^{2}\right)}+\left(\mathrm{D}_{\mathrm{a}} \psi, L_{\Psi_{0}(\tau)} \mathrm{D}_{\mathrm{a}} \psi\right)_{L^{2}\left(\mathbb{R}^{2}\right)} \leq \gamma\|\psi\|_{H^{2}\left(\mathbb{R}^{2}\right)}^{2}$

$$
\gamma^{-1}\|\psi\|_{H^{2}\left(\mathbb{R}^{2}\right)}^{2} \leq\left\|L_{\Psi_{0}(\tau)} \psi\right\|_{L^{2}\left(\mathbb{R}^{2}\right)}^{2} \leq \gamma\|\psi\|_{H^{2}\left(\mathbb{R}^{2}\right)}^{2}
$$

for all $\psi \in H^{2}\left(\mathbb{R}^{2} ; \mathbb{R}^{2} \times \mathbb{C}\right) \cap \operatorname{Ker} L_{\Psi_{0}(\tau)}^{\perp}$.

Proof. The proof appears in appendix B after lemma B.1.

Define

$$
Q(t) \equiv A_{2}\left(\psi(t), L_{\Psi_{0}(\epsilon t)} \psi(t)\right)_{L^{2}\left(\mathbb{R}^{2}\right)}+\left(\mathrm{D}_{\mathrm{a}} \psi(t), L_{\Psi_{0}(\epsilon t)} \mathrm{D}_{\mathrm{a}} \psi(t)\right)_{L^{2}\left(\mathbb{R}^{2}\right)}
$$

for the constant $A_{2}$ to be determined later.

Lemma 5.21. Let $q(\tau)$ satisfy (5.34). Let $T=S / \epsilon$. Then there exists $\Gamma>0$ such that for $0 \leq t \leq T$

$$
\Gamma^{-1}\|\psi(t)\|_{H^{2}}^{2} \leq Q(t) \leq \Gamma\|\psi(t)\|_{H^{2}}^{2} .
$$

Proof. This follows from lemma 5.20.

We now collect together estimates of various error terms in: 
Lemma 5.22. Let $q(\tau)$ satisfy (5.34) and $\epsilon<1$. Then there exists a number $c$ such that at each $t>0$,

$$
\begin{gathered}
\left\|\beta_{0}(t)\right\|_{H^{2}} \leq c\|\psi(t)\|_{L^{2}}\left(\|\dot{\psi}(t)\|_{L^{2}}+\left|q^{\prime}(\epsilon t)\right|\right), \\
\left\|\beta_{0}(t)\right\|_{H^{3}} \leq c\|\psi(t)\|_{H^{1}}\left(\|\dot{\psi}(t)\|_{H^{1}}+\left|q^{\prime}(\epsilon t)\right|\right) .
\end{gathered}
$$

Proof. This follows by elliptic regularity estimates applied to (5.47), using lemma B.1. (Notice that by (5.38) and (5.37) we have a bound $\left\|\Psi_{0}(\tau)\right\|_{W^{1, \infty}} \leq c(K)$ for $0 \leq \tau \leq S$, so we can use the $H^{1}$ norm instead of the $H^{1, a}$ norm defined in (A.1).)

Lemma 5.23. Let $q(\tau)$ satisfy (5.34) then

(i) $M_{t}=\left[L_{\Psi_{0}(\epsilon t)}, \mathrm{D}_{\mathrm{a}}\right]$ is a first order differential operator satisfying

$$
\left\|M_{t} \psi\right\|_{L^{2}} \leq A_{1}\|\psi\|_{H^{1}}
$$

(ii) $\Lambda_{\tau}=\frac{d}{d \tau} L_{\Psi_{0}(\tau)}$ is a first order differential operator satisfying

$$
\left\|\Lambda_{\tau} \psi\right\|_{L^{2}} \leq c\left|q^{\prime}\right|\|\psi\|_{H^{1}}
$$

Proof. The first statement follows directly from calculations using the formulae in appendix B. (Notice that the commutators all give rise to error terms which are curvatures (like $B$ ) or covariant derivatives (like $D \Phi$ ). These are all uniformly bounded ([19])). The second statement follows from (5.37) and (5.38). The same comment about the $H^{1}$ norm applies as in the proof of lemma 5.22 .

Lemma 5.24. Let $q(\tau)$ satisfy (5.34) and assume $Q(t) \leq 2 Q(0)$ for $0 \leq$ $t \leq T=S / \epsilon$. Then there exists positive numbers $\epsilon_{5}$ and $L_{4}>0$ such that for all $\epsilon<\epsilon_{5}$

$$
\begin{aligned}
& \left|q^{\prime}(\epsilon t)\right| \leq \epsilon L_{4} Q(t), \\
& \left\|\beta_{0}(t)\right\|_{H^{2}}^{2}+\|\dot{\psi}(t)\|_{L^{2}}^{2} \leq L_{4} Q(t) .
\end{aligned}
$$

Proof. By lemma 5.21, $Q(t) \leq 2 Q(0)$ implies that $\|\psi(t)\|_{L^{\infty}}$ is bounded for $0 \leq t \leq T$. Using the equation for $q^{\prime}$ in lemma 5.18 and (5.48) we get

$$
\begin{aligned}
\left|q^{\prime}(\tau)\right| & \leq \epsilon c\left(\|\psi\|_{H^{1}}+\left\|\beta_{0}\right\|_{H^{1}}+\|\dot{\psi}\|_{L^{2}}\right) \\
\|\dot{\psi}\|_{L^{2}} & \leq c\left(\|\psi\|_{H^{2}}+\left|q^{\prime}(\tau)\right|+\epsilon\left\|\beta_{0}\right\|_{H^{1}}\right)
\end{aligned}
$$


where $c=c(Q(0))$. Now substitute the result of lemma 5.22 in (5.54). This gives

$$
\left|q^{\prime}(\tau)\right| \leq \epsilon c\left(\|\psi\|_{H^{1}}+\|\dot{\psi}\|_{L^{2}}\right) .
$$

Substitute this into (5.55) and we get the estimate for $\dot{\psi}$. Substituting this into the expression for $\beta_{0}(t)$ in lemma 5.22 and using (5.51) and (5.53) we get the last estimate.

Remark. By making $T$ small we can assume $Q(t) \leq 2 Q(0)$ to be true on a small interval by continuity. It will turn out that $Q$ is nonincreasing for small $\epsilon$ so this assumption will in fact hold with $T=+\infty$. We now obtain exponential decay estimates for $Q(t)$.

Lemma 5.25. Let $A_{2}=10 A_{1}^{2} \gamma$ and assume $0 \leq t \leq T$ so that $Q(t) \leq 2 Q(0)$ and $q(\tau)$ satisfies (5.34). There exist numbers $r_{1}>0, L_{5}>0$ such that for $\epsilon<1$

$$
\dot{Q}+r_{1} Q \leq L_{5}\left(\left|q^{\prime}\right|^{2}+\epsilon^{2}\left\|\beta_{0}\right\|_{H^{2}}^{2}+\epsilon Q\right) .
$$

Proof. Calculate

$$
\begin{aligned}
\dot{Q} & =-2 A_{2}\left\|L_{\Psi_{0}(\tau)} \psi\right\|_{L^{2}}^{2}+\epsilon A_{2}\left(\psi, \Lambda_{\tau} \psi\right)-2 A_{2}\left(\frac{d}{d \tau} \Psi_{0}, L_{\Psi_{0}(\tau)} \psi\right) \\
& +2 \epsilon A_{2}\left(j, L_{\Psi_{0}(\tau)} \psi\right)-2\left\|L_{\Psi_{0}(\tau)} \mathrm{D}_{\mathrm{a}} \psi\right\|_{L^{2}}^{2} \\
& +\epsilon\left(\mathrm{D}_{\mathrm{a}} \psi, \Lambda_{\tau} \mathrm{D}_{\mathrm{a}} \psi\right)-2\left(\frac{d}{d \tau} \mathrm{D}_{\mathrm{a}} \Psi_{0}(\tau), L_{\Psi_{0}(\tau)} \mathrm{D}_{\mathrm{a}} \psi\right) \\
& +\epsilon\left(\mathrm{D}_{\mathrm{a}} j, L_{\Psi_{0}(\tau)} \mathrm{D}_{\mathrm{a}} \psi\right)+\left(L_{\Psi_{0}(\tau)} \mathrm{D}_{\mathrm{a}} \psi, M_{t} \psi\right)
\end{aligned}
$$

where (5.48) and (5.49) are used. All terms but the third, seventh and ninth are estimated as required directly using lemmas 5.19-5.24. The third and seventh terms are estimated using $|(a \cdot b)| \leq \delta|a|^{2}+\frac{1}{4 \delta}|b|^{2}$ for suitable $\delta$. This allows us to absorb them in the first and fifth terms respectively with a remainder which is bounded $\leq c\left|q^{\prime}\right|^{2}$. The final term can be removed by applying Cauchy-Schwarz and recalling $-\left\|L_{\Psi_{0}} \psi\right\|_{L^{2}}^{2} \leq-\gamma^{-1}\|\psi\|_{H^{2}}^{2}$ and lemma 5.23. This shows that it can be absorbed into the first and fifth terms using the definition of $A_{2}$. Indeed this argument gives an estimate for $\left\|L_{\Psi_{0} \mathrm{D}_{\mathrm{a}}} \psi\right\|_{L^{2}}$ and hence from (5.49) we obtain

Corollary 5.26. Under the same conditions as lemma 5.25

$$
\|\dot{\psi}\|_{H^{1}}+\left\|L_{\Psi_{0}(\tau)} \mathrm{D}_{\mathrm{a}} \psi\right\|_{L^{2}} \leq c\left(Q+|\dot{Q}|+\left|q^{\prime}\right|^{2}+\left\|\beta_{0}\right\|_{H^{2}}^{2}\right) .
$$


Lemma 5.27 (Exponential Decay). Let $q(\tau)$ satisfy (5.34) and assume $Q(t) \leq 2 Q(0)$ for $0 \leq t \leq T$. Choose $\epsilon<\epsilon_{6}$ where

$$
\epsilon_{6}=\min \left\{1, \theta, \frac{\epsilon_{2}}{L_{3}}, \epsilon_{4}, \epsilon_{5}, r_{1} /\left(6 L_{5}\right), r_{1} /\left(12 L_{4}^{2} L_{5} Q(0)\right), r_{1} /\left(6 L_{4} L_{5}\right)\right\} .
$$

Then there exist numbers $R, r_{2}$ such that for $0 \leq t \leq T$

$$
\begin{aligned}
& Q(t) \leq Q(0) e^{-r_{2} t} \\
& \left|q^{\prime}(\epsilon t)\right| \leq \epsilon L_{4} Q(0) e^{-r_{2} t} \\
& \left\|\beta_{0}(t)\right\|_{H^{2}}+\|\dot{\psi}(t)\|_{L^{2}} \leq L_{4} Q(0) e^{-r_{2} t} .
\end{aligned}
$$

Proof. By lemma 5.25 and our choice of $\epsilon_{6}$

$$
\dot{Q}+\frac{r_{1}}{2} Q \leq 0 .
$$

From this we find the exponential decay result for $Q(t)$ with $r_{2}=r_{1} / 2$. The other two results follow from lemma 5.24.

Step Five. Conclusion of the Proof It follows from lemma 5.27 that $\exists \epsilon_{7}>0$ such that if $\epsilon<\epsilon_{7}$ then $q(\tau)$ satisfies (5.34) with $S=+\infty$. Taking $K$ small we may assume that $q(\tau)$ lies in the same coordinate chart for all time. From lemmas 5.27 and 5.21 it follows that

$$
\|\psi(t)\|_{H^{2}}^{2} \leq \Gamma Q(t) \leq \Gamma Q(0) e^{-r_{2} t} \leq \Gamma^{2}\|\psi(0)\|_{H^{2}}^{2} e^{-r_{2} t} .
$$

Also it follows from lemma 5.27 and (5.37-5.38) that for some $L_{6}>0$,

$$
\left\|\Psi_{0}(\epsilon t)-\Psi_{0}(0)\right\|_{H^{2}} \leq L_{6} e^{-r_{2} t} .
$$

Therefore $\exists \epsilon_{8}<\min \left\{\epsilon_{6}, \epsilon_{7}\right\}$ such that $\epsilon<\epsilon_{8}$ implies

$$
\begin{array}{cl}
\text { (i) } & \left\|\Psi_{0}(\epsilon t)-\Psi_{0}(0)\right\|_{H^{2}}<\delta_{2} \\
\text { (ii) } & \left\|\Psi^{\#}(t)-\Psi_{0}(0)\right\|_{H^{2}}<\epsilon_{2} \\
\text { (iii) } & \left\|\Psi^{\#}(t)-\Psi_{*}\right\|_{H^{2}}<\theta
\end{array}
$$

for all times. Conditions (i) and (ii) allow us to continue our solution with the gauges specified in step two for all times by lemma 5.9. We can also deduce from (iii) and theorem 5.15 that $\Psi_{0}(\epsilon t)=\mathbb{P}\left(\Psi^{\#}(t)\right)$ for all $t>0$. The solution can now be continued indefinitely and the results of lemma 5.25 apply for $t \in[0,+\infty)$. The curve $q(\tau) \in M_{N}$ will converge to some point $q_{\infty}$ while $\psi \rightarrow 0$ and $\beta_{0} \rightarrow 0$ in $H^{2}$ at an exponential rate. This 
implies $\Psi_{0}(t)$ and $\Psi^{\#}(t)$ will converge in $H^{2}$ to the same limit $\Psi_{\infty}^{\#}$. We now wish to move back to temporal gauge and thus we apply the inverse gauge transformation to that in step two. We obtain $\Psi(t)=\rho(-\chi(t)) \Psi^{\#}(t)$ where $\chi(t)$ was determined in step two. Now by the equivariance of $\mathbb{P}$

$$
\mathbb{P}(\Psi(t))=\mathbb{P}\left(\rho(-\chi(t)) \Psi^{\#}(t)\right)=\rho(-\chi(t)) \Psi_{0}(\epsilon t)
$$

and $\mathbb{P}(\Psi(t)) \in S_{N} \bigcap_{k \in \mathbb{N}} W^{k, \infty}\left(\mathbb{R}^{2} ; \mathbb{R}^{2} \times \mathbb{C}\right)$ by properties (iii) and (iv) of theorem 5.15. Since gauge transformations are isometries in $L^{2}$ we have

$$
\left\|\Psi^{\#}(t)-\Psi_{0}(\epsilon t)\right\|_{L^{2}}=\|\Psi(t)-\mathbb{P}(\Psi(t))\|_{L^{2}}=\inf _{P \in S_{N}^{(2)}}\|\Psi(t)-P\|_{L^{2}}
$$

from (i) of theorem 5.15. Since $\mathbb{P}(\Psi(t)) \in S_{N}^{(2)}$ the uniquness of $\mathbb{P}(\Psi(t))$ follows from theorem 5.15. Therefore to obtain the convergence results in theorem 5.4 we must prove that $\chi(t) \rightarrow \chi(\infty)$ in $H^{3}$. To do this we estimate $|\dot{\psi}|_{H^{1}}$ from corollary 5.26 and then apply lemma 5.22. This implies $\left\|\beta_{0}\right\|_{H^{3}} \leq$ $c e^{-r_{2} t}$ which implies the convergence of $\chi$ since $\epsilon^{2} \beta_{0}=\partial_{t} \chi$. To complete the proof of the theorem we must show that

$$
\inf _{P \in S_{N}}\|\Psi(t)-P\|_{L^{2}}=\inf _{S \in S_{N}^{(2)}}\|\Psi(t)-S\|_{L^{2}} .
$$

Since $\mathbb{P}(\Psi(t)) \in \bigcap_{k \in \mathbb{N}} W^{k, \infty}\left(\mathbb{R}^{2} ; \mathbb{R}^{2} \times \mathbb{C}\right)$ this will follow if we can show that it is impossible that there exists $P \in S_{N}$ such that

$$
\|\Psi(t)-P\|_{L^{2}}<\inf _{S \in S_{N}^{(2)}}\|\Psi(t)-S\|_{L^{2}}
$$

So assume (5.56) holds for $P=Q$; then $Q-\Psi_{*}=Q-\Psi(t)+\Psi(t)-\Psi_{*} \in L^{2}$. But we know from the proof of lemma 5.10 that any $Q \in S_{N}$ is a gauge transformation of $\tilde{Q} \in S_{N}^{(2)}$ by, say, $\rho(\chi)$. Since $Q-\Psi_{*} \in L^{2}$ we have $\chi \in H^{1}$. Let $g_{\nu} \in H^{3}$ satisfy $\left\|g-g_{\nu}\right\|_{H^{1}} \leq 2^{-\nu}$ for $\nu=1,2, \ldots$ Then $S=\rho\left(g_{\nu}\right) \tilde{Q} \in S_{N}^{(2)}$ gives a contradiction to (5.56) for large $\nu$.

\section{Proof of Main Theorem.}

We recall the comments at the beginning of section 5.b. Let $\Psi(t)$ be our solution in the temporal gauge; then first we move into the Coulomb gauge, slightly modified as in theorem 5.3. This gives a fixed gauge transformation $\rho(\chi) \in \cap W^{k, \infty}\left(\mathbb{R}^{2}\right)$, satisfying $d \chi \in \cap_{s=1}^{\infty} H^{s}$ and a point $\Psi_{*} \in$ 
$S_{N} \bigcap_{k \in \mathbb{N}} W^{k, \infty}\left(\mathbb{R}^{2} ; \mathbb{R}^{2} \times \mathbb{C}\right)$ so that at time $T_{0}$ we have $\left\|\rho(\chi) \Psi\left(T_{0}\right)-\Psi_{*}\right\|_{H^{2}}<$ $\epsilon_{*}$. Also $\Psi\left(T_{0}\right) \in \bigcap_{k \in \mathbb{N}} W^{k, \infty}\left(\mathbb{R}^{2} ; \mathbb{R}^{2} \times \mathbb{C}\right)$. We now apply theorem 5.4 to deduce that $\rho(\chi) \Psi(t) \equiv \tilde{\Psi}\left(t-T_{0}\right)$ converges in $H^{2}$ to a limit $\Psi_{\infty}$ at an exponential rate. Therefore since $\chi$ is fixed we can deduce that $\Psi(t)$ will itself converge in $H^{2}$ to a limit $\rho(-\chi) \Psi_{\infty}$, which we relabel as $\Psi_{\infty}$ to complete the proof of the existence of a limit. The exponential decay result follows from the corresponding statement in theorem 5.4 and the fact that $\mathbb{P}$ commutes with the gauge transformations.

\section{A. Appendix A.}

In this section we will give some background material for the convenience of the reader. We will use the following spaces. For complex functions $\eta: \mathbb{R}^{2} \rightarrow \mathbb{C}$ we introduce Sobolev norms given by

$$
\|\eta\|_{r, a}^{2} \equiv \sum_{|M| \leq r}\left\|D_{a}^{M} \eta\right\|_{L^{2}}^{2}
$$

where $D_{a}^{M}$ is the order $M$ covariant derivative and the sum is over all partial indices. Similarly direct sums $\psi=(\beta, \eta): \mathbb{R}^{2} \rightarrow \mathbb{R}^{2} \times \mathbb{C}$ are differentiated according to the direct sum connection

$$
\mathrm{D}_{\mathrm{a}} \psi=\left(\nabla \beta, D_{a} \eta\right) .
$$

We introduce corresponding Sobolev norms:

$$
\|\psi\|_{r, a}^{2} \equiv \sum_{|M| \leq r}\left\|\mathrm{D}_{\mathrm{a}}{ }^{M} \psi\right\|_{L^{2}}^{2} .
$$

By a slight abuse of notation, we will denote the corresponding Sobolev spaces which are defined by completing smooth compactly supported functions with respect to these norms, by $H^{r, a}$ in both these cases. Notice that if $\|a\|_{W^{k, \infty}} \leq M$ then there exists $c=c(n, M)$ such that

$$
c^{-1}\|\psi\|_{n, a}^{2} \leq\|\psi\|_{H^{n}}^{2} \leq c\|\psi\|_{n, a}^{2} .
$$

The space $C^{m, \alpha}\left(\mathbf{R}^{n}\right)$ is the space of (possibly vector valued) functions whose derivatives up to $m^{\text {th }}$ order are continuous, bounded and uniformly Hölder continuous of exponent $\alpha$. Next let $I$ be a closed interval of the $t$-axis. The space $C^{m, \alpha, \beta}\left(\mathbf{R}^{n} \times I\right)$ consists of (vector valued) functions whose spatial derivatives up to $m^{\text {th }}$ order are continuous and bounded and are uniformly 
Hölder continuous with respect to $(x, t)$ with exponents $\alpha, \beta$ respectively. The norms on these spaces $|\cdot|_{m, \alpha, \beta}$ are defined by

$$
\begin{array}{r}
|f|_{m, \alpha} \equiv \sum_{|M| \leq m}\left(\sup _{x, t}\left|\partial^{M} f(x, t)\right|+\sup _{x, y, t} \frac{\left|\partial^{M} f(x, t)-\partial^{M} f(y, t)\right|}{|x-y|^{\alpha}}\right) \\
|f|_{m, \alpha, \beta} \equiv \sum_{|M| \leq m}\left(\sup _{x, t}\left|\partial^{M} f(x, t)\right|\right. \\
+\sup _{x, y, t} \frac{\left|\partial^{M} f(x, t)-\partial^{M} f(y, t)\right|}{|x-y|^{\alpha}} \\
\left.+\sup _{x, t, s} \frac{\left|\partial^{M} f(x, t)-\partial^{M} f(x, s)\right|}{|t-s|^{\beta}}\right)
\end{array}
$$

where $\partial^{M}$ refers to spatial derivatives given by the multi-index $M$. We now have the following local existence theorem for our equation, written in the form given in 2.11 .

Theorem A.1 (Local Existence). Consider the initial value problem

$$
\begin{aligned}
\dot{\Psi} & =\Delta \Psi+f(\Psi, \nabla \Psi) \\
\Psi(x, 0) & =\Psi_{0}(x)
\end{aligned}
$$

with $\Psi_{0} \in C^{2,1}\left(\mathbf{R}^{2}\right)$. Then there exists a time $T_{l o c}=T_{l o c}\left(\left|\partial^{\alpha} \Psi_{0}\right|_{L^{\infty}}|,| \alpha \mid \leq 2\right)$ on which there exists a unique solution $u \in C^{2,1, \frac{1}{2}}\left(\mathbf{R}^{n} \times\left[0, T_{l o c}\right]\right)$.

For the proof of the theorem we refer to [13]. The next theorem concerns the existence of a fundamental solution of linear parabolic systems.

Theorem A.2. Consider a linear parabolic system of the form

$$
\dot{\mathcal{U}}=\Delta \mathcal{U}+f_{1}(x, t) \nabla \mathcal{U}+f_{2}(x, t) \mathcal{U}
$$

where $f_{1}, f_{2}$ are matrix valued functions in the space $C^{0, \alpha, 0}\left(\mathbf{R}^{n} \times[0, T]\right)$, then there exists a fundamental solution matrix $Z(x, y, t, \tau)$ which satisfies estimates

$$
\left|\partial_{x}^{k} Z(x, y, t, \tau)\right| \leq c_{1}(t-\tau)^{-\frac{n+|k|}{2}} e^{-c_{2} \frac{|x-y|^{2}}{t-\tau}} \quad \text { for }|k| \leq 2
$$

where the constants $c_{1}, c_{2}$ depend on $\alpha, T,\left|f_{i}\right|_{0, \alpha, 0}$. This means the solution can be expressed as

$$
\mathcal{U}(x, t)=\int_{0}^{t} \int Z(x, y, t, 0) \mathcal{U}(0, y) d y
$$

and $\lim _{t \rightarrow 0}\|\mathcal{U}(x, t)-\mathcal{U}(x, 0)\|_{L^{\infty}\left(\mathbb{R}^{2}\right)}=0$. 
For the proof see [13].

Corollary A.3. Assume $|\mathcal{U}(x, 0)| \leq c_{3} e^{-r|x|}$. Then $\exists c_{4}(T), R(T)>0$ such that for $t \in[0, T],|x|>R$

$$
|\mathcal{U}(x, t)| \leq c_{4} e^{-\frac{r}{2}|x|}
$$

Proof. We split up the integral in (A.2) into two parts $I_{1}, I_{2}$ on the inner region $I=\left\{|x-y|<\frac{|x|}{2}\right\}$ and outer region $O=\left\{|x-y|>\frac{|x|}{2}\right\}$. Notice that on the inner region $|y|>\frac{|x|}{2}$ so that

$$
\left|I_{1}\right| \leq c_{3} e^{-\frac{r}{2}|x|}|Z|_{L^{1}}
$$

The second part of the integral is estimated as

$$
\begin{array}{r}
\left|I_{2}\right| \leq c_{3} \int_{O} Z(x-y, t) d y \leq c_{3} c_{1} \int_{O} t^{-1} \exp \left(-c_{2} \frac{|x-y|^{2}}{t}\right) \\
\leq c_{5} \exp \left(-c_{2} \frac{|x-y|^{2}}{t}\right),
\end{array}
$$

so choosing $R>\frac{r T}{2 c_{2}}$ gives the result.

We now state a version of the maximum principle from [30]. Let $\Omega$ be an open set in $\mathbb{R}^{n+1}$ with coordinates $(x, t)$. Define for $P \in \Omega$ the set $S(P)$ to be the set of points in $\Omega$ which can be connected to $P$ by a curve along which $t$ is nondecreasing. Let $L$ be an operator of the form

$$
L=\partial_{t}-\sum_{i j} a_{i j}(x, t) \partial_{i} \partial_{j}+\sum_{i} a_{i}(x, t) \partial_{i}+c(x, t)
$$

where the coefficents are continuous and bounded in $\bar{\Omega}$ and satisfy for all $(x, t) \in \Omega$

(i) $\sum_{i j} a_{i j}(x, t) \lambda_{i} \lambda_{j} \geq \delta|\lambda|^{2}, \quad \delta>0$

(ii) $c(x, t) \geq 0$.

Theorem A.4 (Maximum Principle). Let $u \in C^{2}(\Omega)$ satisfy $L u \leq 0$ in $\Omega$. Assume that there exists a point $P_{0} \in \Omega$ such that

$$
\sup _{S\left(P_{0}\right)} u=u\left(P_{0}\right)
$$

Then either $u\left(P_{0}\right)<0$ or $u \equiv u\left(P_{0}\right)$ in $S\left(P_{0}\right)$. 


\section{B. Appendix B.}

In this appendix we summarise a few facts from the paper [37] which are needed. Let $\Psi_{0}=(a, \phi)$ and $\Psi+\psi=(a+\beta, \phi+\eta)$ both be solutions of the Bogomol'nyi equations (5.20-5.21) and assume that $(\beta, \eta)$ satisfies the gauge orthogonality condition with respect to $\Psi_{0}$. Then we find by subtraction that $(\beta, \eta)$ must satisfy

$$
\begin{aligned}
& \overline{\partial_{a}} \eta-\frac{i}{2}\left(\beta_{1}+i \beta_{2}\right) \phi-\frac{i}{2}\left(\beta_{1}+i \beta_{2}\right) \eta=0 \\
& d \beta+(\phi, \eta)+\frac{1}{2}(\eta, \eta)=0 \\
& -d^{*} \beta-(i \phi, \eta)=0 .
\end{aligned}
$$

The part of these equations linear with respect to $(\beta, \eta)$ defines the operator $\mathcal{D}_{\Psi_{0}}$ used in the text. The equations then take the form

$$
\mathcal{D}_{\Psi_{0}} \psi=g(\psi) \quad \text { with } g \text { quadratic. }
$$

Notice that if $\Psi_{0}, \Psi_{0}+\psi$ are static solutions then $|\phi| \leq 1$ and $|\phi+\eta| \leq 1$ and so $\|g(\psi)\|_{L^{2}} \leq c\|\psi\|_{L^{2}}$.

The second order linear operator defined by the Hessian is given by

$$
L_{\Psi_{0}}=\mathcal{D}_{\Psi_{0}}^{*} \mathcal{D}_{\Psi_{0}} .
$$

Explicitly $L_{\Psi_{0}}$ is given by

$$
\begin{aligned}
\left(\psi, L_{\Psi_{0}} \psi\right)_{L^{2}}=\int_{\mathbb{R}^{2}} & |\nabla \beta|^{2}+\left|D_{a} \eta\right|^{2}+|\phi|^{2}\left(|\beta|^{2}+|\eta|^{2}\right) \\
& -2 \beta \cdot\left(\eta, D_{a} \phi\right)-\frac{1}{2}\left(1-|\phi|^{2}\right)|\eta|^{2} .
\end{aligned}
$$

The operator $M_{\Psi_{0}}=\mathcal{D}_{\Psi_{0}} \mathcal{D}_{\Psi_{0}}^{*}$ is given by

$$
\left(\psi, M_{\Psi_{0}} \psi\right)_{L^{2}}=\int_{\mathbb{R}^{2}}|\nabla \beta|^{2}+\left|D_{a} \eta\right|^{2}+|\phi|^{2}\left(|\beta|^{2}+|\eta|^{2}\right) .
$$

Lemma B.1. There exists $\gamma$, independent of $\Psi_{0}$ in $S_{N}$ (or in $S_{N}^{2}$ ), such that for all $\psi \in \operatorname{Ker} L_{\Psi_{0}}^{\perp} \cap H^{1, a}$

$$
\gamma^{-1}\|\psi\|_{1, a}^{2} \leq\left(\psi, L_{\Psi_{0}} \psi\right)_{L^{2}}=\left\|\mathcal{D}_{\Psi_{0}} \psi\right\|_{L^{2}}^{2} \leq \gamma\|\psi\|_{1, a}^{2} .
$$

The quadratic forms $\left(\psi, M_{\Psi_{0}} \psi\right)_{L^{2}}$ and $\int|\nabla u|^{2}+|\phi|^{2}|u|^{2}$ obey estimates similar to (B.2) for all $\psi \in H^{1, a}\left(\mathbb{R}^{2} ; \mathbb{R}^{2} \times \mathbb{C}\right), u \in H^{1, \alpha}\left(\mathbb{R}^{2} ; \mathbb{R}^{2} \times \mathbb{C}\right)$. Since $D_{a} \phi=\nabla \phi-i a \phi$ we can replace the $\{1, a\}$ norm (defined in appendix $A$ ) by the usual $H^{1}$ norm if $|a|_{L^{\infty}}<\infty$ by (A.1). 
Proof. (B.2) in the case of $S_{N}$ is proved in [37, Theorem 3.1]. In the case of $S_{N}^{2}$ apply a gauge transformation to go into Coulomb gauge in which $\Psi_{0}$ is smooth and the norms in (B.2) are invariant after such a gauge transformation (see also the proof of lemma 5.10). The other two quadratic forms are defined by non-negative operators with zero kernel so the proof is obtained as in [37].

Proof of lemma 5.20. By (5.35) we see that $\left\|\Psi_{0}(t)\right\|_{W^{1, \infty}} \leq c<\infty$ and so we can use the $H^{2}$ norm instead of $H^{2, a(\tau)}$ by (A.1). Then (5.52) is obtained by applying (B.2) twice and lemma B.1 implies (5.53) since

$$
\left\|L_{\Psi_{0}} \psi\right\|_{L^{2}}^{2}=\left(\mathcal{D}_{\Psi_{0}} \psi, M_{\Psi_{0}} \mathcal{D}_{\Psi_{0}} \psi\right)_{L^{2}}
$$

Lemma B.2. Let $\phi \in C^{\infty}\left(\mathbb{R}^{2} ; \mathbb{C}\right)$ be bounded and satisfy $\lim _{|x| \rightarrow \infty}|\phi(x)|=$ 1 and $|1-| \phi|| \in L^{1}\left(\mathbb{R}^{2}\right)$. Let $f \in C^{\infty}\left(\mathbb{R}^{2}\right) \cap L^{\infty}\left(\mathbb{R}^{2}\right) \cap L^{2}\left(\mathbb{R}^{2}\right)$ then there exists a unique solution $u \in H^{2}\left(\mathbb{R}^{2}\right) \cap C^{\infty}\left(\mathbb{R}^{2}\right) \cap W^{1, \infty}\left(\mathbb{R}^{2}\right)$ of

$$
\left(-\Delta+|\phi|^{2}\right) u=f
$$

which satisfies a priori estimates

$$
\begin{aligned}
& \|u\|_{H^{2}\left(\mathbb{R}^{2}\right)} \leq c\|f\|_{L^{2}\left(\mathbb{R}^{2}\right)} \\
& \|u\|_{W^{1, \infty}\left(\mathbb{R}^{2}\right)} \leq c\|f\|_{L^{\infty}\left(\mathbb{R}^{2}\right)} .
\end{aligned}
$$

Proof. Existence follows from the Lax Milgram lemma and lemma B.1. The second estimate follows from the representation of the solution in the form

$$
u(x)=\int_{\mathbb{R}^{2}} K(x, y) f(y) d y .
$$

Claim. The kernel $K(x, y)$ is a smooth function of $x$ away from $x=y$, decays exponentially fast to zero as $|x-y| \rightarrow+\infty$ and satisfies $\|K(x, y)\|_{W^{1,1}(d y)} \leq$ $C$ where $C$ is independent of $x$.

Proof of Claim. To prove this notice that $K$ solves

$$
\left(-\Delta_{x}+|\phi(x)|^{2}\right) K(x, y)=\delta(x-y)
$$

so that $K(\cdot, y)$ is strictly positive and satisfies $\int|\phi(x)|^{2}|K(x, y)| d x=1$. It has a logarithmic singularity at $x=y$. To be precise let $\rho(u)$ be a smooth positive radial nonincreasing function equal to one for $|u| \leq 1 / 2$ and equal 
to zero for $|u|>3 / 4$; then $J(x, y)=K(x, y)+\frac{1}{2 \pi} \rho(|x-y|) \ln (|x-y|)$ is bounded in $L^{\infty}(d x)$ independent of $y$. Indeed $J$ satisfies the equation

$$
\left(-\Delta_{x}+|\phi(x)|^{2}\right) J(x, y)=\frac{1}{2 \pi} \rho(|x-y|) \ln (|x-y|)
$$

and the right hand side is bounded in $L^{2}(d x)$ independent of $y$ and therefore $J(x, y)$ is bounded in $H^{2}(d x)$ independent of $y$ and so also in $L^{\infty}(d x)$. By the assumption on $\phi$ we find $K(x, y)$ is bounded in $L^{1}(d x)$ independent of $y$ :

$$
\begin{aligned}
\int K(x, y) d x & \leq\left.\int|1-| \phi(x)\right|^{2}|K(x, y)| d x+\int|\phi(x)|^{2}|K(x, y)| d x \\
& \leq\left.\|J(x, y)\|_{L^{\infty}(d x)} \int|1-| \phi(x)\right|^{2} \mid d x \\
& \quad+c \int\left|\frac{1}{2 \pi} \rho(|x-y|) \ln (|x-y|)\right| d x \\
& \quad+\int|\phi(x)|^{2}|K(x, y)| d x \\
& \leq c\left(1+\|J(x, y)\|_{L^{\infty}(d y)}\right) .
\end{aligned}
$$

Self-adjointness implies $K(x, y)=K(y, x)$ and so $K(x, y)$ is also bounded in $L^{1}(d y)$ independent of $x$. Having proved this we can infer that the same is true of $\partial_{x} K(x, y)$ by differentiation of the equation for $J$ and applying the $L^{1}$ boundedness of the operator $f \mapsto \int K(\cdot, y) f(y) d y([14,6.3])$. Thus we deduce that $\partial_{x} K(x, y)$ is bounded in $L^{1}(d y)$ independent of $x$. The result now follows immediately.

Lemma B.3. Assume $\Psi_{0} \in \bigcap_{k \in \mathbb{N}} W^{k, \infty}\left(\mathbb{R}^{2} ; \mathbb{R}^{2} \times \mathbb{C}\right)$ and let $q \mapsto \Psi_{0}+\psi(q)$ be a local family of solutions as in lemma 5.11 for $q$ in some small compact set $\Sigma$ inside the chart provided by the local diffeomorphism $F$ in the lemma. Then $\left\|\partial_{q} \psi\right\|_{W^{m, \infty}} \leq\left\|\partial_{q} \psi\right\|_{H^{m+2}} \leq c(m, \Sigma)<\infty$ for all $m=1,2, \ldots$

Proof. By the construction in lemma 5.11 we know that $\left\|\partial_{q} \psi\right\|_{H^{2}} \leq c$. Differentiate (B.1) for $\psi$ to get

$$
\mathcal{D}_{\Psi_{0}} \partial_{q} \psi=\partial_{q}(g(\psi))
$$

If we apply the covariant derivative $D_{a}$ defined in appendix $A$ to this we find

$$
\mathcal{D}_{\Psi_{0}}\left(\mathrm{D}_{\mathrm{a}}\left(\partial_{q} \psi\right)\right)=\left[\mathcal{D}_{\Psi_{0}}, \mathrm{D}_{\mathrm{a}}\right] \partial_{q} \psi+\mathrm{D}_{\mathrm{a}} \partial_{q}(g(\psi)) .
$$


The error term is a commutator and so contains curvature terms which can be estimated a priori ([19]). They are in $W^{k, \infty}$ for all $k$. The same structure occurs if $\mathrm{D}_{\mathrm{a}}$ is applied again. We therefore get the estimate $\left\|\mathrm{D}_{\mathrm{a}}{ }^{\alpha} \partial_{q} \psi\right\|_{1, a} \leq$ $c(|\alpha|, \Sigma)$ for all multi-indices $\alpha$. Since $\Psi_{0} \in \bigcap_{k \in \mathbb{N}} W^{k, \infty}\left(\mathbb{R}^{2} ; \mathbb{R}^{2} \times \mathbb{C}\right)$ this implies $\left\|\partial_{q} \psi\right\|_{H^{m+2}} \leq c(m, \Sigma)$ by (A.1).

Proof of lemma 5.10 We define a map Coul $: S_{N}^{(2)} \rightarrow S_{N}$ by moving into Coulomb gauge: given $\Psi_{0}=(a, \phi) \in S_{N}^{(2)}$ we solve for $\Psi=(A+$ $\left.d \chi, \Phi e^{i \chi}\right)$ satisfying the elliptic system (3.14) with $B=\partial_{1} a_{2}-\partial_{2} a_{1}$. This will give a smooth solution ([19]) and so maps into $S_{N}$. (This procedure is unique only up to gauge transformation by harmonic functions; thus to fix Coul we require that $\lim _{|x| \rightarrow \infty}|A(x)|=0$ and $\lim _{x_{1} \rightarrow \infty} \Phi\left(x_{1}, 0\right)=+1$, where $x_{1}, x_{2}$ are coordinates on the plane. The first condition cuts down the indeterminacy to gauge transformation by constants which is then removed by the second condition.) The gauge transformation $\chi$ is determined by solving $\nabla \chi=A-a$ which will be in $H_{l o c}^{3}$ as $A-a \in H_{l o c}^{2}$.

We now show that this induces a map $C: M_{N}^{(2)} \rightarrow M_{N}$ which is a bijection. First of all to see that it is well defined consider $(A, \Phi)$ and $\left(A^{\mathrm{b}}, \Phi^{\mathrm{b}}\right)$ in $S_{N}^{(2)}$ such that $\pi(A, \Phi)=\pi\left(A^{\mathrm{b}}, \Phi^{\mathrm{b}}\right)$, i.e.

$$
\left(A^{\mathrm{b}}, \Phi^{\mathrm{b}}\right)=\left(A+d g, \Phi e^{i g}\right) \text { for some } g \in H^{3} .
$$

We must show that

$$
\pi(\operatorname{Coul}(A, \Phi))=\pi\left(\operatorname{Coul}\left(A^{b}, \Phi^{b}\right)\right)
$$

Now $\operatorname{Coul}(A, \Phi)=(\tilde{A}, \tilde{\Phi})=\left(A+d \chi, \Phi e^{i \chi}\right)$ and $\operatorname{Coul}\left(A^{b}, \Phi^{b}\right)=\left(\tilde{A}^{b}, \tilde{\Phi}^{b}\right)=$ $\left(A^{b}+d \chi^{b}, \Phi^{b} e^{i \chi^{b}}\right)$ where

$$
\Delta \chi+\nabla \cdot A=0 \quad \text { and } \quad \Delta \chi^{b}+\nabla \cdot A^{b}=0 .
$$

Then let $h=g-\chi+\chi^{b} \in H_{l o c}^{3}$ and we have $\rho(h)(\tilde{A}, \tilde{\Phi})=\left(\tilde{A}^{b}, \tilde{\Phi}^{b}\right)$. But since $\nabla \cdot \tilde{A}=\nabla \cdot \tilde{A}^{b}=0$ it follows that $h \in C^{\infty}$ which implies (B.3). Thus Coul induces a map on equivalence classes, which we denote by $C$.

We next show that the map $C$ is onto. Pick any $\Psi \in S_{N}$ and (since $M_{N}$ is connected) a curve $\gamma(\tau)$ from $\pi\left(\Psi_{*}\right)$ to $\pi(\Psi)$. Then by lemma 5.13 we lift this to a horizontal curve starting at $\Psi_{*}$ and given by

$$
\Psi_{*}+\int\left(\frac{d}{d \tau} \gamma_{\mu}\right) n_{\mu}(\gamma(\tau)) d \tau
$$


The endpoint $\Psi^{b}$ of this curve will satisfy $\pi(\Psi)=\pi\left(\Psi^{b}\right)$ and $\Psi^{b} \in \mathcal{A}^{(2)}$ by (5.38).

Finally we show that $C$ gives a one-to-one map. Thus consider $(A, \Phi)$ and $\left(A^{b}, \Phi^{b}\right)$ in $S_{N}^{(2)}$ such that $(A, \Phi) \notin \mathcal{G}^{(3)} \cdot\left(A^{b}, \Phi^{b}\right)$ but such that $\rho(g)(\tilde{A}, \tilde{\Phi})=$ $\left(\tilde{A}^{b}, \tilde{\Phi}^{b}\right)$ for $g \in C^{\infty}$. The gauge transformations $\chi, \chi^{b}$ are in $H_{\text {loc }}^{3}$ so there exists $h \in H_{l o c}^{3}$ such that $\rho(h)(A, \Phi)=\left(A^{b}, \Phi^{b}\right)$. Therefore since $(A, \Phi)$ and $\left(A^{\mathrm{b}}=d h+A, \Phi^{\mathrm{b}}=e^{i h} \Phi\right)$ are in $A^{(2)}$ it follows that $d h \in H^{2}$. But also since $\left(\Phi-\Phi^{b}\right) \in H^{2}$ and $\lim _{|x| \rightarrow \infty}|\Phi(x)|=1$ we see that $h \in L^{2}$ and so $h \in H^{3}$ which completes the proof.

\section{Appendix C.}

Proof of lemma 5.14 Let $F=\left(f_{1}, f_{2}\right)$, then $\psi=F-\Psi_{*}$ solves the first order elliptic equation

$$
\mathcal{D}_{\Psi_{*}} \psi=g(\psi)+h(\psi)
$$

where $g$ is as in lemma 5.11 and appendix $\mathrm{B}$, and $h$ is given by

$$
h=\left(0, \nabla \cdot\left(A-a_{*}\right)+\left(i \phi_{*}, \Phi-\phi_{*}\right)+\left(i\left(\Phi-\phi_{*}\right), f_{2}-\phi_{*}\right)\right) .
$$

This latter term is present because $\psi \notin \mathcal{S} \mathcal{L}_{\Psi_{*}}^{(2)}$. It follows on account of the inequalities $|\phi(x)| \leq 1,|\Phi(x)| \leq 1$ that $g, h$ satisfy

$$
\begin{aligned}
& \|g(\psi)\|_{L^{2}}+\|h(\psi)\|_{L^{2}} \leq c\left(1+\|\psi\|_{L^{2}}\right) \\
& \|g(\psi)\|_{H^{1}}+\|h(\psi)\|_{H^{1}} \leq c\left(1+\|\psi\|_{H^{1}}\right)
\end{aligned}
$$

Therefore the elliptic regularity estimate in appendix B implies $\|\psi\|_{H^{1}} \leq$ $c\|\psi\|_{L^{2}}$. A single bootstrap completes the proof of the lemma.

Proof of lemma 5.16 Write $\Psi=(A, \Phi)$ and $\Psi_{0}=(a, \phi)$. We wish to consider

$$
\inf \left\{I(\chi): \chi \in H^{3}\left(\mathbb{R}^{2}\right)\right\}
$$

where

$$
I(\chi)=\int\left(|A-a-d \chi|^{2}+\left|\Phi-\phi e^{i \chi}\right|^{2}\right) d x .
$$

Write $s=s(\chi)=e^{i \chi}-1$ and consider the minimization

$$
\inf \left\{\tilde{I}(s): s \in H^{3}\left(\mathbb{R}^{2} ; \mathbb{C}\right) \text { and }|1+s|=1 \text { everywhere }\right\}
$$


where

$$
\tilde{I}(s)=\int\left(\left|A-a+i(1+s)^{-1} d s\right|^{2}+|\Phi-\phi-\phi s|^{2}\right) d x .
$$

These two problems are equivalent for two reasons:

(i) $I(\chi)=I(s(\chi))$.

(ii) The function $\chi \mapsto s(\chi)$ defines a bijection between $\left\{\chi \in H^{3}\left(\mathbb{R}^{2} ; \mathbb{R}\right)\right\}$ and the set

$$
\Sigma=\left\{s \in H^{3}\left(\mathbb{R}^{2} ; \mathbb{C}\right):|1+s(x)|=1 \text { everywhere }\right\} .
$$

Indeed we can define an inverse to $s$ as follows: given $s(x)$ satisfying these conditions define

$$
\chi(x)=-i \int_{0}^{x}(1+s)^{-1} d s+c_{1}
$$

where $\chi(0) \in[0,2 \pi)$ satisfies $s(0)=e^{i \chi(0)}-1$ and the real constant $c_{1}$ is to be defined later. (The condition $|1+s(0)|=1$ and $c_{1} \in \mathbb{R}$ ensure that $|1+s(x)|=1$ everywhere). Notice that the integrand in this path integral is $C^{1}$ and by Stokes' theorem (applied to the real and imaginary parts) the result is independent of the path taken. The resulting function will satisfy $d \chi \in H^{2}$, so that $\chi$ is uniformly continuous and by integration we find that

$$
s(x)=e^{i\left(\chi(x)-c_{2}\right)}-1
$$

for some constant $c_{2}$ determined freely by $c_{1}$. Since $\chi$ is uniformly continuous and $s \in L^{2}$ it follows that $\lim _{|x| \rightarrow \infty}\left|\chi(x)-c_{2}\right|=2 n \pi$ where $n$ is an integer. By appropriate choice of $c_{1}$ we ensure $c_{2}=0$. But then $\operatorname{since} \sin (\chi) \in L^{2}$ we get $\chi \in L^{2}$. Therefore the constant $c_{1}$ can be chosen such that $\chi \in L^{2}$ and thus also $\chi \in H^{3}$.

We will now show that these minimisation problems have a solution. Since $|s(x)| \leq 2 \forall x$ and $\lim _{|x| \rightarrow \infty}|\phi(x)|=1$ the functional $\tilde{I}$ is coercive with respect to the $H^{1}$ norm, i.e.,

$$
\tilde{I}(s) \geq c_{1}\|s\|_{H^{1}}^{2}-c_{2} .
$$

By the convexity of the integrand in the argument $d s, \tilde{I}(s)$ is weakly sequentially lower semicontinuous in $H^{1}$ and hence attains its infimum on $H^{1}$. Let $\left(s_{n}\right)_{n \geq 1} \in H^{3}\left(\mathbb{R}^{2} ; \mathbb{C}\right)$ with $s_{n}=e^{i \chi_{n}}-1$ be a minimising sequence for $\tilde{I}(s)$ which by coercivity is bounded in $H^{1}$ norm. (By the density theorem proved in [2] we may assume that $s_{n} \in H^{3}$ ). Extract a subsequence (not relabeled) weakly convergent in $H^{1}$ to a limit $s$. By Rellich's theorem it will converge strongly in $L_{l o c}^{2}\left(\mathbb{R}^{2}\right)$ and so, at least subsequentially, we can assume 
$s_{n}(x) \rightarrow s(x)$ for $x$ a.e. in $\mathbb{R}^{2}$ (this being true initially for every compact subset of $\mathbb{R}^{2}$ is true on all of the plane by a diagonal argument using an increasing sequence of compacts). Hence $|1+s(x)|=1$ a.e. To show the regularity of this minimiser it is convenient to write this in terms of $\chi$.

Claim There exists a function $\chi$ in the space

$$
X=\left\{\chi: d \chi \in L^{2}, \sin \chi \in L^{2},(1-\cos \chi) \in L^{2}\right\}
$$

which minimises $I$ over the space of all such functions.

To prove this notice that by Poincare's inequality and Rellich's lemma there exist, for any $R>0$, numbers $k_{n}$ such that $\chi_{n}-k_{n}$ are strongly convergent in $L^{2}\left(B_{R}\right)$; to be precise let $k_{n}\left|B_{R}\right|=\int_{B_{R}} \chi_{n}$. Since $\|\cdot\|_{L^{2}\left(B_{R}\right)} \leq\|\cdot\|_{L^{2}\left(B_{S}\right)}$ for $R \leq S$ we can, by a diagonal argument, obtain a subsequence and a sequence of real numbers $c_{n}$ such that $\chi_{n}-c_{n}$ converge in $L^{2}(K)$ for any compact set $K$. Call the limiting function $\chi(x)$. But recall also that $e^{i \chi_{n}}-1$ converges also strongly in $L^{2}(K)$ for compact $K$ and therefore $c_{n} \rightarrow 0 \bmod 2 \pi \mathbf{Z}$ and $s=e^{i \chi}-1$. Also $d \chi_{n} \rightarrow d \chi$ weakly in $L^{2}$ and so it follows (by lower semicontinuity) that

$$
I(\chi) \leq \liminf I\left(\chi_{n}\right) \quad \text { and } \quad I(\chi)=\inf _{X} I .
$$

This completes the proof of the claim.

Taking smooth variations at $\chi$, i.e., $\left.\frac{d}{d \lambda}\right|_{\lambda=0} I(\chi+\lambda \zeta)=0$ for all $\zeta$ smooth and compactly supported we obtain, in the sense of distributions, the following Euler-Lagrange equation:

$$
-\Delta \chi=\left(i \Phi, \eta e^{i \chi}\right)+\left(i \Phi, \Phi\left(e^{i \chi}-1\right)\right)-\nabla \cdot \beta
$$

where $\Psi=\Psi_{0}+(\beta, \eta)$. The right hand side lies in $H^{1}\left(\mathbb{R}^{2}\right)$ which implies that $\|\chi\|_{H^{3}(B(x, R)}$ is bounded uniformly in $x$. Therefore $d \chi \in L^{\infty}$ and so since $(1-\cos \chi), \sin \chi \in L^{2}$ we deduce that $\lim _{|x| \rightarrow \infty} \chi(x)=0$ (after possibly adjusting $\chi$ by $2 n \pi$ for some interger $n$ ) and $\chi \in L^{2}$. Thus $\chi, s$ both lie in $H^{3}$ and the two original minimisation problems have been solved. Finally the $W^{k, \infty}$ statement follows from lemma B.2.

Proof of lemma 5.17. Step One. We introduce the set

$$
X \equiv\left\{F=\left(f_{1}, f_{2}\right) \in \mathcal{A}^{(2)}: \mathcal{B}(F)=0 \text { and }(F-\Psi) \in \mathcal{S} \mathcal{L}_{\Psi}^{(2)}\right\} \subset \mathcal{A}^{(2)}
$$

with the induced topology. Here $\mathcal{B}$ is the set of solutions of the Bogomol'nyi equations given in (5.20-5.21). This is a sequentially weakly closed subset of $\mathcal{A}^{(2)}$. We will minimise the function $d: X \rightarrow \mathbb{R}^{+}$given by

$$
d(F)=\|F-\Psi\|_{L^{2}}
$$


This is a sequentially weakly lower semi-continuous function.

Step Two. Claim A. The function $d$ is coercive i.e. there exist positive constants $c_{1}, c_{2}$ such that

$$
d(F) \geq c_{1}\left\|F-\Psi_{*}\right\|_{H^{2}}^{2}-c_{2} .
$$

Proof of Claim. First notice that the Hilbert space inequality $2|(a, b)| \leq$ $\frac{1}{2}|a|^{2}+2|b|^{2}$ implies $d(F) \geq \frac{1}{2}\left\|F-\Psi_{*}\right\|_{L^{2}}^{2}-2 \epsilon^{2}$ so it remains to prove

$$
\left\|F-\Psi_{*}\right\|_{L^{2}} \geq c\left\|F-\Psi_{*}\right\|_{H^{2}}-\left\|\Psi-\Psi_{*}\right\|_{H^{2}} .
$$

This follows from lemma 5.14 and the result follows since $\Psi-\Psi_{*} \in H^{2}$.

Step Three. Claim $B$ The function $d$ attains a minimum at some point $\Psi_{0} \in X$ and $d\left(\Psi_{0}\right) \leq L_{3} \epsilon$ where $L_{3}=1+L_{2}$, where $L_{2}$ is as in lemma 5.9.

Proof of Claim Since $\left(\Psi_{0}-\Psi\right) \in \mathcal{S L}_{\Psi}^{(2)}$ it follows from remark (ii) following definition 1 that $\left(\Psi_{0}-\Psi\right) \in \mathcal{S} \mathcal{L}_{\Psi_{0}}^{(2)}$. From lemma 5.9 there exists $\tilde{\Psi}_{*}$, gauge equivalent to $\Psi_{*}$, such that $\tilde{\Psi}_{*}-\Psi \in \mathcal{S} \mathcal{L}_{\Psi}^{(2)}$ and this will satisfy

$$
\left\|\Psi_{*}-\tilde{\Psi}_{*}\right\|_{H^{2}} \leq L_{2} \epsilon .
$$

Therefore

$$
\begin{aligned}
d\left(\Psi_{0}\right) & =\left\|\Psi_{0}-\Psi\right\|_{L^{2}\left(\mathbb{R}^{2}\right)} \\
& \leq\left\|\tilde{\Psi}_{*}-\Psi\right\|_{L^{2}\left(\mathbb{R}^{2}\right)} \\
& \leq\left\|\tilde{\Psi}_{*}-\Psi_{*}\right\|_{H^{2}\left(\mathbb{R}^{2}\right)}+\left\|\Psi_{*}-\Psi\right\|_{H^{2}\left(\mathbb{R}^{2}\right)} \\
& \leq \leq\left(1+L_{2}\right) \epsilon .
\end{aligned}
$$

Step Four. Claim $C \Psi_{0} \in \bigcap_{k \in \mathrm{N}} W^{k, \infty}\left(\mathbb{R}^{2} ; \mathbb{R}^{2} \times \mathbb{C}\right)$.

Proof of Claim By (C.4) we have $\left\|\Psi_{0}-\Psi_{*}\right\|_{H^{2}} \leq c \epsilon$. Therefore for small enough $\epsilon$ we can apply lemma 5.7 to gauge transform $\Psi_{0}$ onto the local solution space $\tilde{M}_{N}\left(\Psi_{*}\right)$. It then follows by lemmas B.3 and 5.7 and from the assumption $\Psi_{*} \in \bigcap_{k \in \mathbb{N}} W^{k, \infty}\left(\mathbb{R}^{2} ; \mathbb{R}^{2} \times \mathbb{C}\right)$ that $\Psi_{0} \in \bigcap_{k \in \mathbb{N}} W^{k, \infty}\left(\mathbb{R}^{2} ; \mathbb{R}^{2} \times \mathbb{C}\right)$.

Step Five. Now consider the local solution space $\tilde{M}_{N}\left(\Psi_{0}\right)$. Choose a local coordinate system $q$ in $M_{N}$ around $\pi\left(\Psi_{0}\right)$ with $q=0$ corresponding to $\Psi_{0}$, so that $q \mapsto \tilde{\Psi}_{0}(q) \in \tilde{M}_{N}\left(\Psi_{0}\right)$ is a local diffeomorphism with $\Psi_{0}(0)=\Psi_{0}$ as in lemma 5.11. Then lemma 5.9 provides us with gauge transformations $\chi(q), \chi(0) \equiv 1$ which give a diffeomorphism $\tilde{\Psi}_{0}(q) \rightarrow \rho(\chi(q)) \tilde{\Psi}_{0}(q)$ between neighbourhoods of $\Psi_{0}$ in $\tilde{M}_{N}\left(\Psi_{0}\right)$ and in $X$. (This incidentally shows that $X$ is a smooth submanifold in a neighbourhood of $\Psi_{0}$.) Now define a function of $q$ given by $\tilde{d}(q) \equiv d\left(\rho(\chi(q)) \tilde{\Psi}_{0}(q)\right)$ which is smooth and takes on a minimum 
at $q=0$. Therefore $\tilde{d}^{\prime}(0)=0$. To complete the proof recall that $\left(\Psi-\Psi_{0}\right) \in$ $\mathcal{S} \mathcal{L}_{\Psi_{0}}^{(2)}$ so that

$$
\left(\Psi-\Psi_{0}, \frac{\partial}{\partial q_{\mu}}\left(\rho(q) \Psi_{0}(0)\right)\right)_{L^{2}}=0
$$

Therefore

$$
\tilde{d}^{\prime}(0)=\left(\Psi-\Psi_{0}, \frac{\partial}{\partial q_{\mu}} \tilde{\Psi}_{0}(q)\right)_{L^{2}}=0
$$

which in turn implies $\left(\Psi-\Psi_{0}\right)$ is $L^{2}$ orthogonal to $\operatorname{Ker} L_{\Psi_{0}}$ since $\left(\Psi-\Psi_{0}\right) \in$ $\mathcal{S} \mathcal{L}_{\Psi_{0}}^{(2)}$

\section{References.}

[1] P. Bauman and C-N. Chen and D. Phillips and P. Sternberg (1995), Vortex Annihilation in Nonlinear Heat Flow for Ginzburg-Landau Systems, Euro. J. Appl. Math., 6, 115-126.

[2] F. Bethuel and X. Zheng (1988), Density of smooth functions between two manifolds in Sobolev spaces, J. Funct. Anal., 80, 60-75.

[3] N. Hitchin (1988), Geometry and topology of Moduli Spaces, Springer lecture Notes in Mathematics, 1451, 1-48.

[4] C. Elliott and H. Matano and T. Qi (1994), Zeros of complex Ginzburg-Landau order parameter with applications to superconductivity, Euro. J. Appl. Math., $5,431-448$.

[5] S. Chapman, S. Howison and J. Ockendon (1992), Macroscopic Models for Superconductivity, SIAM Review, 34(4), 529-560.

[6] M. Berger and Y. Chen (1989), Symmetric Vortices for the Ginzberg-Landau Equations of superconductivity and the nonlinear desingularisation phenomenon, J. Functional Analysis, 82, 259-295.

[7] J. Hale and G. Raugel (1992), Convergence in gradient-like systems with applications to PDE, ZAMP, 43,1, 63-124.

[8] H. Brezis and F. Merle and T. Riviere, Quantisation effects for $-\Delta u=u(1-$ $\left.|u|^{2}\right)$.

[9] E.B. Bogomol'nyi (1976), Stability of Classical Solutions, Sov. Journal of Nuclear Physics, 24, 861-870. 
[10] Q. Du and M. Gunzburger and J. Peterson (1992), Analysis and Approximation of the Ginzburg-Landau model of Super conductivity, Siam Review, 34, 45-81.

[11] S. Donaldson and P. Kronheimer (1990), The Geometry of Four-Manifolds, Oxford University Press, Oxford.

[12] J. Dieudonne (1972), Treatise on Analysis, vol. III, Academic Press, Oxford.

[13] S. Eidel'man (1969), Parabolic Systems, North Holland, London.

[14] G.B. Folland (1984), Real Analysis, Wiley, New York.

[15] D. Freed and K. Uhlenbeck (1984), Instantons and Four Manifolds, Springer, New York.

[16] R. Hamilton (1988), The Ricci Flow on Surfaces, Mathematics and General Relativity, Contemp. Math. Vol. 71, Birkhauser, Basel, ed. J. Isenberg.

[17] L. Hormander (1983), The analysis of linear partial differential operators, Springer, Berlin.

[18] A. Hassell (1993), The Yang-Mills Heat Flow on $\mathbf{R}^{3}$, J. Funct. Anal., 111, 431-448.

[19] A. Jaffe and C. Taubes (1982), Vortices and Monopoles, Birkhauser, Boston, Mass.

[20] T. Kato (1980), Perturbation theory for Linear Operators, Springer, New York.

[21] T. Kato (1958), Perturbation Theory for nullity, deficiency and other quantities of linear operators, J. d'Analyse Math., 6, 261-322.

[22] F. Bethuel and F. Helein and H. Brezis (1994), Ginburg-Landau Vortices, Birkhauser, Boston, Mass.

[23] L. Jacobs and C. Rebbi (1979), Interaction of superconducting vortices, Physical review B, 19, 4486-4494.

[24] S. Lang (1985), Differential Manifolds, Springer, New York.

[25] F. H. Lin (1996), Some dynamical properties of Ginzburg-Landau Vortices, Commun. Pure Appl. Math., XLIX,4, 323-364.

[26] A. J. Majda (1984), Compressible Fluid Flow and Systems of Conservation Laws, Springer, New York.

[27] N. Manton (1982), A remark on scattering of BPS monopoles, Physics Letters, 110B, 54-56. 
[28] B. Plohr, Existence, Regularity and Behaviour of Isotropic Solutions of Classical Gauge Field Theories, Princeton University, 1980.

[29] J. Rade (1992), On the Yang-Mills Heat Equation in Two and Three Dimensions, J. reine angew. Math., 431, 123-163.

[30] L. Nirenberg (1953), A strong maximum principle for parabolic equations, CPAM, 6, 167-177.

[31] Y.N. Ovchinnikov and I. Sigal, Ginsburg-Landau equation I. Static vortices, University of Toronto, preprint, 1995.

[32] P. Mironescu (1995), On the stability of radial solutions of the GinzburgLandau equation, J . Funct. Anal., 130, 334-344.

[33] E. Lieb and M. Loss (1994), Symmetry of the Ginzburg-Landau minimiser, Math. Res. Lett., 1, 701-715.

[34] T. Samols (1992), Vortex scattering, Commun. Math. Phys., 145, 149-180.

[35] P. Shah, Vortex Scattering at Near Critical Coupling, To appear in Nuclear Physics B.

[36] L. Simon (1983), Asymptotics for a Class of Evolution Equations, with Applications to Geometric Problems, Ann. Math., 118, 525-571.

[37] D. Stuart (1994), Dynamics of Abelian Higgs vortices in the near Bogomolny regime, Commun. Math. Phys., 159, 51-91.

[38] D. Stuart (1994), The Geodesic Approximation for the Yang-Mills-Higgs equations, Commun. Math. Phys., 166, 149-191.

[39] D. Stuart (1996), Interaction of Superconducting Vortices and Asymptotics of Ginzburg-Landau FlowW, Appl. Math. Lett., 9,5, 27-33.

[40] M. Weinstein and J. Xin, Dynamic Stability of the Vortex Solutions of the Ginzburg-Landau and Nonlinear Schrodinger Equations, Preprint,1995.

RECEIVED MAY 23, 1995.

[January 26, 1996]

DAMTP

UNIVERSITY OF CAMBRIDGE

CAMBRIDGE CB3 9EW, ENGLAND

UNIVERSITY OF CALIFORNIA, DAVIS

DAVIS, CA 95616, U.S.A.

S.DEMOULINI@DAMTP.CAM.AC.UK

D.STUART@DAMTP.CAM.AC.UK 